\title{
MOSQUITO REPELLENTS
}

\author{
BEING A REPORT OF THE WORK OF THE MOSQUITO \\ REPELLENT INQUIRY, CAMBRIDGE 1943-5
}

\author{
By Sir S. RICKARD CHRISTOPHERS, C.I.E., O.B.E., F.R.S., I.M.S. (RETd.) \\ Zoological Laboratory, University of Cambridge
}

(With 6 Figures in the Text)

\section{CONTENTS}

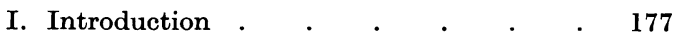

II. Historical . $\quad . \quad$. $\quad . \quad$. 178

(1) Early researches on repellents . . 178

(2) Developments during the war . . 180

(3) History of testing of repellents . 182

III. Repellent compounds and their preparations $\quad . \quad . \quad . \quad . \quad . \quad . \quad . \quad 182$

(1) Repellent compounds $\quad$. $\quad . \quad 182$

(2) Preparations of repellent compounds 185

IV. Technique of rearing standardized cultures of Aedes aegypti . . . . . $\quad$. 186

(1) Requirements .

(2) Obtaining and storing the eggs $\quad$. 190

(3) Hatching out . . . . . . 191

(4) Rearing to the pupa . . . . . 191

(5) Collection of pupae . . . . $\quad$. 192

(6) Emergence $\quad . \quad$. . . . . $\quad 192$

(7) Transferring to the test cage . . 192

V. Technique of laboratory testing for repellency . . . . . . . . $\quad . \quad 193$

(1) Requirements . $\quad . \quad$. $\quad . \quad$. 193

(2) Preliminary observations . . . 194

(3) Preparing the arm . . . . . 195

(4) Preparing the test cage $\quad$. $\quad . \quad$. 195

(5) Performing the 'control' . . . 195

(6) Performing the test . . . . . 196

(7) Assessment of results $\quad$. $\quad$. $\quad . \quad 196$

VI. Compounds and their preparations tested by the Unit

(1) Compounds $\quad \cdot \quad \cdot \quad \cdot 197$

$\begin{array}{lllllll}\text { (2) Preparations } & \cdot & \cdot & \cdot & \cdot & \cdot & 197\end{array}$

VII. Observations directed to a 'rub-resistant' DMP cream . $\quad . \quad$. $\quad$. $\quad$. 204

(1) Considerations involved . . $\quad . \quad 204$

(2) China clay as a basis for DMP creams 205

(3) Demonstration of rate of disappearance of DMP from skin by the use of clay films

(4) Result of applying repellent preparations in different ways . . .

(5) General conclusions regarding rubresistant creams .
VIII. Physical properties in relation to repellency . . . . . . $\quad .208$

(1) Boiling-point . $\quad . \quad$. $\quad . \quad 208$

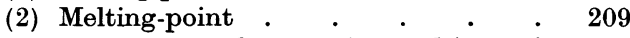

(3) Viscosity, surface tension and 'creep' 209

(4) Specific gravity . . . . 211

IX. On an apparatus for measuring repellency at a distance $\quad . \quad . \quad . \quad . \quad . \quad 211$

(1) The apparatus . $\quad . \quad$. $\quad . \quad 211$

(2) Method of use . $\quad . \quad$. $\quad . \quad$. $\quad 213$

(3) Effect of heat, moisture and sex . 214

(4) Effect of different repellents . . 215

(5) General considerations . . . 215

X. Impregnation of fabrics . . . . . 216

(1) Method of testing $\quad$. $\quad$. $\quad . \quad 2 \quad 216$

(2) Protection period given by different repellents $\quad . \quad$. $\quad . \quad$. $\quad 217$

(3) Effect of nature of fabric . . . 217

(4) Impregnation of netting . . . 218

XI. Penetrability of fabrics to mosquito bites

(1) Technique - $\cdot$ -

(2) Structural features of fabrics in relation to penetrability . $\quad . \quad 219$

(3) Type of fabric . $\quad . \quad$. $\quad . \quad$. 221

(4) Mechanism of penetration . . 221

XII. Summary and conclusions . . . . 223

XIII. Acknowledgements $\quad$. $\quad$ • $\quad$ • $\quad 225$

List of works dealing with mosquito repellency $\quad . \quad . \quad . \quad . \quad . \quad . \quad 226$

A. References to repellency : . 226

B. References having relation to laboratory use of Aedes aegypti .

Appendices
A. Statistical study of data provided
by 'controls' and 'normal feeds'

B. Note by Dr E. A. Moelwyn-Hughes on conversion of boiling:points .
C. Note by Mr J. R. Whinfield on elementary cloth geometry
218

218 


\section{INTRODUCTION}

There does not appear to exist any published recent general account of the nature of and method of testing repellents and their preparations against mosquitoes. The present report has therefore largely been compiled with the object of giving at least a general idea of the present position in these respects.

A really satisfying account is scarcely possible since the essential cause of repellency and such questions as the relation of molecular structure to repellency are largely unexplored. There has also been a large volume of work carried out during the war in the United States, Canada, Australia, India and in this country, which it is clearly impossible to give in detail even if war restrictions did not come into the question. However, the Unit's work has covered most of the recent developments relating to repellents against mosquitoes as will beclear from the list of contents which heads the report and thus should give a general idea of most of the issues involved.

A considerable amount of experience has also been gained in the testing of fabrics for penetrability to mosquito bites, a work in practice closely related to the testing of repellents, and as little will be found in the literature on this subject it has been dealt with in some detail.

It is scarcely necessary to say that large numbers of compounds show some, or even a considerable, degree of repellent effect. To determine the relative effectiveness of compounds and their preparations requires therefore some form of test that to be satisfactory should give quantitative and repeatable results. It was largely due to absence of these characters in the tests employed in much early work on repellents that the relative merits of different substances, in spite of much work done, remained very uncertain and accounts as to their efficacy often conflicting.

Two methods of final assessment are open to be followed, viz. (1) repetition of tests until massed results finally give some form of answer, and (2) the use of strictly standardized methods so that the same result can be obtained within narrow limits on every occasion. The second method is that towards which efforts of the Unit have been consistently directed and with a large measure of success. It is no use, however, standardizing technique of testing if the mosquitoes used behave differently on different occasions. Hence vital to any effort to arrive at standardized testing is the ability to produce standardized cultures of mosquitoes.

The species of mosquito used by the Unit has throughout been Aedes aegypti. Circumstances made it impractical for serious use of Anopheles to be made, and in so far as species of Anopheles may exhibit differences from Aedes in their reactions to repellents this is to be regretted. It does not, however, invalidate results obtained relative to A. aegypti, which is a species having many advantages for standardized testing. It is essential, however, if the species is to yield repeatable results, that the technique of rearing and every step up to and including the performance of the tests must be strictly standardized, and the only way to ensure this is to breed and maintain the species under fixed optimum conditions.

Success in this respect has been largely dependent on many details, learnt as a result of experience, combined in a routine which has been developed and which has led to great economy of labour and mental energy. To be useful, therefore, description of the technique used must necessarily be given in detail and as a system, even though it is recognized the details given may not apply under different circumstances.

As regards the technique used in testing, results depend so largely upon the amount of repellent applied that quantitative methods are essential. The maximum application that can usually be made of an oily compound is about $20 \mathrm{cu} . \mathrm{mm}$./sq.in. and the minimum that can be uniformly spread without a diluent about 5 cu.mm. (roughly about 1.5 and $0.5 \mathrm{ml}$. for the whole surface of the forearm). For testing in the first place whether a substance is a repellent or not, a dose of 10 cu.mm./sq.in. $(0 \cdot 3 \mathrm{ml}$. to the area of 30 sq.in. marked out on the back of the forearm and exposed in the metal shield used) has usually been used. If definite repellency is exhibited in this dosage tests are repeated using 5 cu.mm./sq.in. or in special cases even smaller doses using a diluent. Creams are measured by a special device which is described.

Of great importance is the time limit of effectiveness. Many substances which might repel immediately after application, e.g. alcohol, can scarcely be said to be repellent, and some reasonable time must be decided upon as a minimum at which it can be considered that repellency is displayed. This period as adopted by the Unit has been $2 \mathrm{hr}$. A compound which for any reason in suitable dosage is ineffective at $2 \mathrm{hr}$. from application can have no practical use as a repellent and is desirably excluded from such category, except perhaps in some special form of investigation. This criterion, it may be observed, excludes any substance that will have entirely disappeared due to volatility by this time, an important consideration dealt with in § VIII when dealing with the boiling-point.

It has been usual to employ as a measure of duration of effectiveness the time in minutes to the first bite. There are, however, serious objections to such a criterion, except perhaps where a merely preliminary screening of large numbers of compounds is being carried out. Even in this case it is doubtful if it is the most convenient, economical or 
reliable method. The nature of the objections to it are briefly noted in the section on technique of testing. The method that has been employed by the Unit is one based on giving an exposure of $15 \mathrm{~min}$. duration at 2,4 and $6 \mathrm{hr}$., or in special cases 3,6 and $9 \mathrm{hr}$., using for each test a fresh cage, and making an assessment on the results. The method of assessment is fully described later.

Suitability of compounds and their preparations for use as repellents is dependent on other characters than their essential repellency and duration of effectiveness. Thus the ease with which they can be smeared on the skin, the extent to which they are liable to seep or be rubbed off or absorbed by the skin, their possible irritant effect or even toxicity if absorbed are all important.

Also important are certain physical characters of compounds, e.g. the boiling-point or the meltingpoint in the case of solids. Boiling-point is particularly important, not only in giving a very useful clue to the probable staying power of a repellent, but also because in the synthesis of new repellents it may be possible by a substitution in the molecule, e.g. the introduction of an $\mathrm{OH}$ group, to raise the boiling-point and thus convert an active but too volatile compound into a good repellent. A difficulty with high boiling-points is that these are often of necessity given as determined under low pressures so that it is difficult to compare one with another. A method of coping with this difficulty with suffcient accuracy for present purposes will be found as an appendix. Viscosity is another physical character which has some importance and on which some results are given in the report.

Characters may have importance in relation to the particular purpose which repellents are intended to serve. Thus for military purposes great stress is laid on long duration of effectiveness, whereas for civilian use 2 or $3 \mathrm{hr}$. would probably meet all requirements. Smell is fatal for repellents intended to be used in jungle warfare, but provided it is pleasant it may even be an advantage in civilian use. Owing to the importance attached to long duration of effectiveness for military purposes research on repellents during the war has tended to develop a type of repellent with very high boiling-point and hence, almost as a corollary, less effective at a distance than some more volatile repellents. Criteria quite other than those given prominence in war might therefore considerably modify search for new synthetic repellents required in peace for civilian use.

The relation of molecular constitution to repellency is very little known, but some types of compounds appear specially liable to exhibit repellent properties and some remarks in this connexion will be found in the report.

Regarding the physiological basis of repellency nothing is known.

\section{HISTORICAL}

(1) Early researches on repellents

Until the recent war substances employed to protect against mosquitoes were mostly of the nature of household remedies. The most widely used repellent, as it would now be called, has undoubtedly been oil of citronella, used either as the oil itself, or as an ingredient in various mixtures, e.g. with liquid paraffin and coconut oil as 'Bamber oil', as an ointment in petroleum jelly (Dover's preparation) or in some other preparation. Perhaps the other most commonly used preventive in the tropics are the cones or sticks (joss-sticks), probably containing pyrethrum, burnt by the housewife to 'drive away mosquitoes'.

Among the first to summarize what was known of such early remedies was Howard (1917) in U.S. Dep. Agric., Farmer's Bull. no. 444, replaced in 1928 by Bull. 1570 in the same series by Howard \& Bishopp, this latter publication representing the position up to the date noted so far as the use of repellents in practice was concerned. The authors distinguish substances (repellents) applied to the skin of exposed parts or used on the clothing, smudges or smokes, fumigants, sprays, the planting of eucalyptus and other vegetation supposed to banish mosquitoes, and larvicides for destroying the larvae in their breeding places.

The first authors to carry out laboratory investigation in this field appear to have been Bacot \& Talbot (1919). They tested various substances and preparations of these in regard to their repellent effect upon $A$. aegypti. They found oil of cassia, mixtures containing oil of citronella, a light wood oil and a proprietary preparation 'parasitox' repellent. They conclude, however, that it is useless to expect protection for longer periods than a few hours. They inclined to the view that repellent effect may be more due to obscuring human smell than by direct deterrent effect of the substance.

A very thorough study of the reactions of mosquitoes (chiefly A. sollicitans and other wild Aedes) was made by Rudolfs (1922-4). The mosquitoes were little attracted by such substances as human sweat, sebaceous secretions, blood, etc., though phenyla. lanine and haemoglobin had some attractiveness and several amino-acids induced them to suck. They were activated (exhibited excitement) by $\mathrm{CO}_{2}$, ammonia, temperature and human breath. They were repelled by oleic acid, valeric acid, lactic acid, indol $0.1 \%$ and skatol $0.1 \%$, though in later papers these were recorded as little effective.

In later observations the author tested some 90 substances, including essential oils, heavy oils, solvents and tar products, esters and other compounds. The figures given for many of these substances varied considerably from one series of tests to another, but 
the following were considered the most effective, viz. pyrethrum (in petroleum) which gave 100 $120 \mathrm{~min}$. protection, oil of thuja $75 \mathrm{~min}$., citral 60-80 min., cinnamic aldehyde 50-60 min. (but burnt the skin), anisic aldehyde $60 \mathrm{~min}$.

A great many other essential oils, pennyroyal, clove, lime citronella, rose geranium, bitter orange, nutmeg, bitter almond, thyme, peppermint, spearmint, eucalyptus, juniper berries, rosemary, wintergreen, laurel, also various pine-like oils, such as oil of hemlock pine, oil of pine needles, spruce oil, oil of pini pumilionis and tar were also found effective, though at the best protection rarely exceeded an hour.' Oil of bergamot, aniseed, lemon, lavender, ginger, sassafras and cedar gave poor results, as also did turpentine, kerosene, phenol, quinoline, crude petroleum and other substances including ethyl and other esters, alcohols (fusel oil, menthol, eucalyptol) and some organic acids. Some of these substances were noted as burning the skin (cinnamic aldehyde, cinnamon (cassia), peppermint, methyl salicylate) or to be otherwise unfit for use (tar). Some 18 solvents (chloroform, ether, methyl, propylic and amyl alcohol, nitrotoluene, benzaldehyde, carbon tetrachloride, $o$ - and $p$-dichlorobenzol, xylol) were tested and most found repellent, though no time is given except the remark against many that repellency did not last long. It is obvious that many of these latter substances would have volatilized in a very short time. The author considered that directly repellent substances were of more value than those which were thought to work through masking the body odour.

The results of some other early workers in this direction may also be briefly mentioned. Echstein (1920) seems to have been the first to draw attention to the fact that different species of mosquito react differently to any given repellent. Fletcher (1920) concluded that different factors are concerned in repulsion at a distance and on contact and that in neither case is repellency proportional to the poisonous effect on the mosquito. Given (1922) found oil of citronella used straight by personnel on board a naval vessel extremely effective, $10 \mathrm{lb}$. lasted the whole summer and most of the ship's company used it. Half a drachm or less in the palm of the hand was sufficient to anoint the feet, ankles, hands and face and to render these parts immune for at least $4 \mathrm{hr}$. and in the author's experience for the whole night. This author also found joss-sticks smouldering under the table very effective causing mosquitoes to fall stupified on the floor. The smell of the fumes in the room was sufficient to drive mosquitoes out or keep them away. Fowler (1926) reports on a repellent ointment, vermijelly and Oxford grease, issued to troops in Palestine. Vermijelly appeared the most effective, Oxford grease was too dirty a material for the purpose. Coogle (1923, 1925) recommended creosote as a repellent applied to the walls and ceilings of houses. Fermi (1926), however, who tried this method found it ineffective. Freeborn (1928) gives a formula consisting of oil of citronella 3 oz., spirits of camphor $1 \mathrm{oz}$., oil of tar $1 \mathrm{oz}$., oil of pennyroyal $\frac{1}{4} \mathrm{oz}$. and castor oil $4 \mathrm{oz}$. as the most effective for use against Sierran Aedes. Its effectiveness lasted half an hour. Cooley et al. (1928) tested a number of repellents for the use of persons in the field, but the protection afforded was temporary. A mixture of equal parts of oil of citronella with some oil that is non-irritating to the skin, such as olive oil, was as effective as any.

Bunker \& Hirschfelder (1925) attempted to arrive at a chemical basis for repellent effect. The tests were carried out against Culex quinquefasciatus, Psorophora, Aedes taeniorrhynchus and other species. The use of reared mosquitoes was not found satisfactory. They note that it was difficult to interpret the results, but of the substances tested they give citronellol as the most effective.

Their most important work lay, however, in the attempt to associate repellency with molecular constitution. They tested various hydrocarbons, alcohols, aldehydes, ketones, ethers and esters. Effectiveness seemed to enter with the $O$ atom, especially with the $\mathrm{COH}$ of the alcohols or the $\mathrm{COO}$ of the esters or the $\mathrm{CO}$ of ketones and aldehydes.

Throughout the next decade we mostly find oil of citronella and sometimes other essential oils being used in some form or another and the first indications of the use of pyrethrum in the form of kerosene extract as a repellent. Dover (1930) recommends the following formula which has been widely used in India: citronella oil (Burgoyne's) $\frac{1}{2}$ oz., spirits of camphor $\frac{1}{4}$ oz., cedarwood oil $\frac{1}{4}$ oz., and white petroleum jelly $2 \mathrm{oz}$. Mail (1934) tested 20 substances used by Bunker \& Hirschfelder. The best results were obtained with citronellol. Moore (1934) suggests that repellency increases in the order hydrocarbon-alcohol-ester and that the best material is a very slightly volatile unsaturated cyclic ester. Ginsburg (1935) found spraying of herbage in limited areas with pyrethrum extract in kerosene $(0.9 \%$ pyrethrins) gave temporary protection against mosquitoes in gatherings in the open. Macnay (1938) found a mixture of oil of thyme $\frac{1}{2} \mathrm{oz}$., concentrated pyrethrum extract $\left(\frac{1}{5} \mathrm{lb}\right.$. flowers per oz.) and castor oil 2-3 oz. effective for 3-5 hr. when used by foresters and field workers. Granett (1940) greatly developed methods of exact testing (see later) and made butyl carbitol acetate the standard against which other repellents were tested. Roy, Ghosh \& Chopra (1942) compared the efficacy of different culicifuges under laboratory conditions against Anopheles stephensi, Armigeres obturbans, Aedes aegypti and Culex fatigans. They obtained protection for 2-3 hr. against Aedes aegypti with a mixture containing oil of citronella and pyrocide 20 
(a pyrethrum preparation), also with a similar mixture using lemon grass oil.

Little is known of influences attracting mosquitoes. Perhaps the most outstanding contribution in this respect is that by Crum (1922). Like Rudolfs this author found that crude mixtures of the components of perspiration and of blood produced only faint erratic response. It was otherwise with warmth. Air bubbled through warm water to a funnel placed near the netting of a cage containing Culex pipiens caused the mosquitoes to thrust their proboscis through the netting so that viewed sideways the appearance resembled that of a beard. Response occurred between $90^{\circ}$ and $160^{\circ} \mathrm{F}$. $\left(32-43^{\circ} \mathrm{C}\right.$.). Breath had the same effect. The mosquitoes were attracted to cloth moistened with warm water but not with cold. The belief that mosquitoes are attracted by human smell seems to have been widely taken for granted, but there seems to be no proof of this.

There seems little doubt but that with Aedes aegypti it is heat (warm convection currents) which is the main directive for this species. The surprising thing is their sensitiveness to such stimuli which approaches that of a special sense. In cage experiments, where up to 20 or more out of 100 mosquitoes will have settled to feed on the arm within 15 sec., no notice is taken of the arm if under glass until the glass warms. When a warm hand is placed against the glass side of a cage the mosquitoes are attracted, not to the hand, but to a zone above the hand where it is evident that warm, convection currents are being set up as the glass warms.

It is characteristic of much of the work in this early period that the substances tested were not pure chemicals, but for the most part natural oils, etc., the composition of which is liable to much variation. In spite of much work the relative repellent powers of different substances were left very uncertain. It was not until the war made the subject of great practical importance that the testing of synthetic organic compounds was undertaken on a large scale.

\section{(2) Developments during the war}

Whilst there has been a great deal of research upon repellents during the war and great advances made in knowledge of these substances, very little has been published and records relating to such work are almost entirely confined to unpublished communications issued under war restriction regulations. By far the largest contribution has come from work carried out in the United States and especially that originating from the Orlando Institute, Florida, where many thousands of synthetic compounds have been tested. But much important work has also been carried out under the military and other authorities in this country, in Canada, the Middle
East, India and Australia. Adequate reference to all these sources in such a note as the present would be impracticable even if permissable and only a broad outline giving the chief results can be attempted.

The stage to which knowledge of repellents had reached on the outbreak of the war has already been indicated. When the urgent necessity came of providing some effective repellent preparation for the use of the forces operating in mosquito infested and malarious areas recourse was naturally first made to preparations containing oil of citronella. Unsatisfactory protection given soon led to the search for more effective substances. Indications seem to point to pyrethrum as most likely to give the desired result. This was especially so since at the time its use had been much facilitated by the development of methods for determining the content of the active principle (pyrethrins I and II) and the existence of highly concentrated extracts containing up to $40 \%$ of these components. Various preparations, creams, tragacanth pastes, etc., containing up to $1 \%$ pyrethrins, though at first seemingly promising, were found to be, though highly lethal, ineffective as repellents. Various substances tested at this time, such as coumarin, vanillin, ethylene and diethylene glycol, 'staway' (diethylene glycol monobutyl and monoethyl) ether acetate, lethane and other insecticidal substances also failed eventually to become established as effective repellents.

It was at this stage that as a result of testing numerous synthetic compounds in the search for active repellents at Orlando two substances were found of outstanding effectiveness, viz. indalone, a proprietary preparation, and Rutger's 612 (2-ethylhexane-1:3-diol) one of a number of compounds synthesized at Rutger's University. In preliminary trials in the laboratory indalone gave an average protection time of $133 \mathrm{~min}$., the range in 10 tests being between 47 and 243 , and a rating of 128 , the rating being the degree of effectiveness compared with that of butyl carbitol acetate* as 100. In the field the rating averaged 154 against $A$. sollicitans and 136 against $A$. taeniorrhynchus. Rutger's 612 gave an average protection period of $352 \mathrm{~min}$. ( $6 \mathrm{hr}$.) with a range of $183-485 \mathrm{~min}$. In the field the straight substance was effective for $325 \mathrm{~min}$.

At the Second Congress on Insect Repellents held in November 1942 it was reported that, in addition to these two repellents, tests were then adequate to recommend the use of a third compound, viz. dimethyl phthalate.

These three substances are those that have for various reasons been selected and since used for the American Forces, a mixture of all three in the pro-

* The compound on which Granett (1940) based his technique. 
portions, dimethyl phthalate 6, Rutger's 6122 and indalone 2, being considered especially effective for all-round work. Indalone has never had the status, as determined in later trials, of the other two compounds, Rutger's 612 has also not been available in any dégree outside America. But dimethyl phthalate has been widely used throughout a number of theatres of war and may be said to be the repellent of the day. It is as efficient, or nearly so, as any other compound known, it is in large supply and easily manufactured, it is stable, very suited to application and of about the right volatility for a lasting repellent (b.p. $282-285^{\circ}$ C.), it is colourless and does not stain clothes and it has only slight smell. There is every reason to believe that it is harmless either as regards any serious irritant effect or toxic liability.

It would, however, be incorrect to think that these were the only effective substances discovered, or that repellency is a property confined to a few synthetic compounds. Many other equally, if not in some cases more, effective compounds exist and some degree of repellency is a very common property. A discussion of the results obtained at Orlando is, as already stated, not attempted, but as an indication of the variety of compounds exhibiting this property the following list of those which have been recorded as giving a protection period of over $300 \mathrm{~min}$. may be given:

$n$-(n-amyl) succinimide $n$-butyl-dl-malate

$n$-capric acid isopropyl cinnamate citronellic acid di-butyl phthalate $N$ - $N$-di-isoamyl acetamide di-methyl phthalate

Testing at Orlando has been carried out not only against Aedes aegypti, but extensively against Anopheles quadrimaculatus. As a rule effectiveness against Anopheles is lower than against Aedes. In this respect dimethyl phthalate appears to be more effective against Anopheles than Rutger's 612 and indalone (Mr Knipling in review of the work at Orlando, 6th Cong. Insect Repellents held 11 May 1944).

Testing of compounds to determine the extent they are absorbed by the skin or give rise to irritant or toxic effects has been extensively carried out in the United States by Dr Calverly and his collaborators. Such testing on various laboratory animals has taken into consideration not only acute but also chronic toxicity, the latter obviously being important where repellents are to be used over long periods. Both dimethyl phthalate and Rutger's 612 have as a result of such enquiries been shown to be innocuous.

Apart from testing in the laboratory it is important that repellents and various preparations of these should be tried out under conditions in which they are likely to be used in practice. Thus the effect of sweating and of rubbing by clothing may greatly reduce the effective period, different species of mosquito under various natural conditions may react very differently and so on. It is also necessary to know how personnel use repellents and what forms of preparation are preferred and also to know what methods of application should be recommended. A great deal of such field and semi-field work has been carried out during the war under the military authorities in this country and in the States and particularly in Canada, the Middle East, India and Australia. Thus D. F. Waterhouse (Med. Reps. from Australia) found Ceylon oil of citronella gave only 20-30 min. protection as against $45 \mathrm{~min}$. or over by dimethyl phthalate, but it kept mosquitoes away even from unprotected parts. Dover's cream gave almost no protection. Pyrethrum gave consistently poor protection, although toxic effect on mosquitoes biting was observed. 'Staway' lotion and butyl carbitol acetate (its main ingredient) both gave fairly good results. Indalone was variable, but even the best results were not comparable with those from dimethyl phthalate. Rutger's 612 gave the same average protection time as dimethyl phthalate. Some slight irritation with Rutger's 612 was, however, noted with tender or sun-burned skin. Some local essential oils (oil of huon pine and three others) gave better protection than oil of citronella. The repellency from huon pine oil and another local oil was ascertained to be due to methyl eugenol. Coumarin (saturated soluble in alcohol) gave fair protection in cage tests, leaving a coating of white crystals with pleasant smell and being non-irritant. $25 \%$ creosote in kerosene gave no protection, nor did eucalyptus or sassafras oil, napthalin or furfural. The author calls attention to the fact that some substances (e.g. Rutger's 612) continue to be considerably repellent even after the first bite has occurred, whilst others (e.g. Staway) break down rapidly from this point.

Lastly no account of recent developments would be complete without some mention of the use of repellents, especially dimethyl phthalate and dibutyl phthalate, for the impregnation of clothing. Clothing so impregnated retains its repellency for long periods, a matter of weeks. Even smearing of clothing with dimethyl phthalate by hand has been shown to be effective. Impregnation can be carried out by spraying the clothing, or by the use of emulsions or solvents into which the clothing is dipped and wrung out. Even impregnated wide-mesh netting has been found to afford protection (unpublished reports by Brig. J. A. Sinton and Major C. G. Johnson) and has been put into practice and found effective in India. 


\section{(3) History of testing of repellents and their preparations}

Testing of repellents has usually been carried out (1) in the field with wild mosquitoes under natural conditions, and (2) in the laboratory.

Whilst the first method might seem that most adapted to give the information desired, since it most nearly approaches the conditions in which a repellent is to be used, it has serious drawbacks in practice. Not only may the number of mosquitoes biting on any particular occasion vary greatly, but also the fierceness with which the mosquitoes attack is very dependent on the species and the atmospheric and other conditions. Thus tests cannot be relied upon as being strictly comparable, with the result that it is very difficult accurately to compare the effectiveness of one substance tested with another.

Unfortunately much of the early work on repellents was restricted to tests in the field and such laboratory tests as were undertaken were little adapted to give accurate results.

The first to attempt to introduce definitely quantitative values in testing was Granett (1940). In laboratory tests he used cages with approximately 500 mosquitoes (mostly Aedes sollicitans and $A$. cantator). The untreated arm was first exposed in the cage, usually for $2 \mathrm{~min}$. and the biting frequency or rate of biting per minute determined. The treated arm was then introduced for an equal period, the procedure being repeated every 15 or $30 \mathrm{~min}$. until several bites had been received on the treated arm. The time taken to the first bite on the treated arm was termed the repellent time. The repellent rating was the repellent time for the substance tested as compared with that of a standard substance taken as 100 , at the same biting frequency.

The standard substance used by Granett was Staway lotion (butyl carbitol acetate). For field tests a similar procedure was adapted exposing the arm to the elbow or the leg to the knee. $1 \mathrm{ml}$. of the substance was used for the arm and $2 \mathrm{ml}$. for the leg. A very similar technique based on that of Granett has been in use at the Orlando Institute.

Though at first sight such a criterion as time to first bite seems unobjectionable there are some serious defects to its use in practice which should be mentioned. In the first place it does not allow for adequate distinction between degree of repellency and its duration. Thus some substances may be very powerfully repellent, but due to volatility or other causes have a short duration of effectiveness. Others may not be so effective as to stop a single bite or so but last much longer. Some substances when they begin to fail break down rapidly, whereas others continue to be reasonably effective for some considerable time after. Again, time to first bite does not give so much information about such matters as is desirable. Apart, however, from such considerations the first bite is too variable an occurrence to lay such great stress upon. There are other objections which come out particularly in practice. Thus when performing tests under laboratory conditions mosquitoes usually settle and bite much more within the first few minutes of exposure than later when they have had more opportunity to become aware that the treated area is objectionable. Further, when using the same cage over and over again, conditions as compared with the first exposure are no longer comparable.

It is true that the method is much more adapted to field tests, the difficulty here being the proviso 'at the same biting rate'. It is doubtful, however, whether field tests are the most suitable form of test for precise determination of the essential efficacy of different repellents. Their place would seem to be that of putting to practical test results found in the laboratory and extending such results to include the effect of various modifying conditions inherent in practical use of repellents. Methods of testing and of assessment of results are dealt with later in $\S \mathrm{V}$.

\section{REPELLENT COMPOUNDS AND THEIR PREPARATIONS}

\section{(1) Repellent compounds}

A brief description of the more important compounds which have been used as repellents, or might be so used, may be useful. The following are such descriptions.

\section{Oil of citronella}

Oil of citronella is one of the Indian grass oils which include also lemon grass oil, palmarossa oil, ginger grass oil and vetiver or cus-cus oil. Up to the outbreak of the recent war it was the most widely used of all repellents against mosquitoes. According to Granett (1940) one of the earliest to recommend its use was J. B. Smith (1901).

There are two recognized forms of the oil, viz. Java oil and Ceylon oil. The former is considered much superior to and purer than the latter, which is, or was formerly, heavily adulterated with kerosene up to a point to enable it to puss a particular test (Schimmel's test).

The chief components are geraniol (16-45\%) and citronellal $(17-34 \%)$, but $d$-citronellol, citral and other compounds of this general type are also present. Quality of the oil was formerly judged by Schimmel's test (dilution with alcohol), but the oil is now increasingly assayed on the geraniol standard, i.e. total acetylizable constituents. Since citronellal is almost certainly the chief active agent giving it repellent properties its content of citronellal would seem the most desirable point to ascertain when to be used as a repellent. 
Oil of citronella (Java oil) gives good protection under laboratory conditions for $2 \mathrm{hr}$. or longer. It has the property of repelling at a distance, giving an index in this respect of +45 as against +1.5 for dimethyl phthalate.* For this reason it is much more likely to 'drive away' mosquitoes than the high boiling-point modern repellents.

Citronellal, the active agent, occurs in two forms, viz. as citronellal and rhodinal; ordinary citronellal is a mixture of these. Citronellol the corresponding alcohol has a boiling-point of $118^{\circ} \mathrm{C} .17 \mathrm{~mm}$. (equiv. approx. to $247^{\circ} \mathrm{C} . / 760 \mathrm{~mm}$. $\dagger$ ). It is a strong repellent with considerable lasting powers. It occurs in the $l$-form in rose oil and in the $d$-form in citronella oil. (For further particulars Parry (1922) and Nelson \& Russell (1925) may be consulted.)

\section{Other essential oils}

Most essential oils consist of varying proportions of certain types of compound, viz. unsaturated terpenes (limonene, dipentine, etc.), unsaturated alcohols (geraniol, citronellol, etc.), aldehydes (citral, citronellal, cinnamic aldehyde, etc.), ketones (menthone, pulegone), phenolic compounds (eugenol, thymol, carvacrol, etc.) and esters of these. The following list gives the chief components of those that appear to have shown exceptional repellent properties. Oil of cassia and vetiver oil tested in this Unit gave results approximating to those of Java citronella oil :

\section{Oil of citronella}

Lemon grass oil

Palmarossa oil

Ginger grass oils

Oil of cassia

Oil of pennyroyal

Oil of thyme

Clove oil

Pine needle oil

Huon oil

\section{Hydroxycitronellal}

Though, so far as is known to the writer, this compound has not been in use as a repellent it has a special interest as an example of a compound of essential oil type the boiling-point of which has been raised by substitution of an $\mathrm{OH}$ group in the molecule giving it greater lasting power, thus being suggestive of possible new effective repellents of this type. It is a powerful repellent with action at a distance. It has a very pleasant smell, being used as a perfume and as a fixative in perfumery. It is an oily liquid.

\footnotetext{
* See § IX.

$\dagger$ See $\S$ VIII.
}

\section{Coumarin, vanillin}

Coumarin is a phenolic acid anhydride (unsaturated). Vanillin is the methyl ester of a phenolic aldehyde. Coumarin has been shown to possess definite repellent properties. It is a solid (m.p. 68-70 ${ }^{\circ} \mathrm{C}$.) with pleasant smell (of new mown hay). It can be applied as a lotion in a solvent leaving a crystalline deposit on the skin with pleasant effect. Vanillin has a melting-point of $81 \cdot 5-82 \cdot 5^{\circ} \mathrm{C}$. It has some repellent effect.

\section{Pyrethrum}

Obtained from the flowers of several species of chrysanthemum, but chiefly from Chrysanthemum cinerariifolium. Formerly obtained mainly from Japan and Dalmatia, but latterly from Kenya and possibly other sources. Can be grown as a crop in England.

The active principles are two esters of pyrethralone with a mono- and di-carboxylic acid (chrysanthemic acid) respectively, viz. pyrethrin $I$ and pyrethrin II. Pyrethrin I is steam distillable, pyrethrum II is not.

Pyrethrum is a powerful and extremely rapid contact insecticide, both the pyrethrins being actively lethal. How far pyrethrum, as thought at one time, is repellent to mosquitoes is rather difficult to decide owing to the extreme rapidity of its toxic effect. There has also been some difference of opinion as to how far it acts other than on contact. In § IX some observations are given which appear to show that in pure concentrated form (a highly concentrated sample of pyrethrin I and of pyrethrin II very kindly supplied by Dr T. F. West of Messrs Stafford Allen and Sons, Ltd.) pyrethrum I does produce some toxic effect without contact, though very much less than on contact, whilst pyrethrin II was entirely without such effect. In both cases when the nearly pure substances were used repellent effect was, even with pyrethrin I, very slight. It may also be noted that in spite of early claims as an effective repellent by some authors it has in later work in the United States, Canada and Australia been given a low grading in this respect.

Pyrethrum was formerly commonly used as a kerosene oil extract the strength of which was stated in terms of the amount of flowers used in making it. Since the outbreak of the war it has been available in the form of highly concentrated extracts containing up to $40 \%$ pyrethrins $(20$ and $40 \%$ colourless extracts; Stafford Allen and Sons) and preparations containing it are now always made to some stated percentage of pyrethrins. It is usual in preparations to include an anti-oxidant, such as phenol, as preservative and also to combine it with a synergist to increase its effect and thereby ensure economy in its use. Synergists that have been used are, among others, 
oil of sesame (not all oils of sesame are, however, effective) and isobutyl undecilenamide (IN 930).

Pyrethrum though not irritant when applied to the skin has been shown to cause sensitization in some cases with resultant serious effects if then used. This is not the case with the purified pyrethrins.

\section{Staway (Staway Insect repellent lotion)}

A proprietary preparation based on extensive testing of organic chemicals and proprietary preparations by Granett (1940) (Rutger's University).

It consists of a mixture of diethylene glycol monobutyl ether acetate (butyl carbitol acetate) and diethylene glycol monoethyl ether (together $65 \%$ ) with $7 \%$ corn oil and $28 \%$ alcohol.

It has been given the rating by Granett (1940) of 100 as against 65 for oil of citronella, at the time the most effective available repellent. Its chief active principle, butyl carbitol acetate, has been used by the Orlando Institute investigators as the standard for comparison with other repellents, the 'rating' being given for such as against 100 for butyl carbitol acetate, as used by Granett.

It has given good results as a repellent to a number of observers, but its use has been discontinued for United States Forces due to possible toxicity.

\section{Indalone}

A proprietary preparation manufactured by United States Industrial Chemicals Inc. It is used as 'Refined indalone' for reasons given below.

It is described as dihydropyrene, or $\alpha \alpha$-dimethyl- $\alpha$ carbutory-dehydro- $\gamma$-pyrone (also as $n$-butyl mesityl oxide acetate). It is not a pure substance and contains a small amount of dibutyl oxalate which is highly toxic and applied to the intact skin of the rabbit causes severe kidney damage. Refined indalone as supplied for the American Forces is guaranteed to contain less than $1 \%$ of thissubstance.

Indalone is a slightly coloured thin oily rather mobile liquid with a faint 'burnt' smell. It stains clothing and like other repellents of its class is a paint solvent. It has strong repellent properties but, as generally agreed, is inferior in activity to dimethyl phthalate or Rutger's 612. It is poorly absorbed by the skin and causes no systemic reactions. It appears to be most effective when used 'straight'. It seems to act chiefly as a contact repellent and in tests, when not too strong, exhibits in a high degree the curious phenomenon of mosquitoes alighting, leaving and re-alighting ('hopping').

It is mostly in use as a component of the $6: 2: 2$ mixture (dimethyl phthalate 6 parts, Rutger's 612 2 parts, indalone 2 parts).

Rutger's 612 (2-ethylhexane-1:3-diol)

Is on the market as a proprietary preparation 'Eveready Insect Repellent, formula 612' (National
Carbon Co. Inc., New York). It is not a pure substance being prepared by simple hydrogenation of butyraldol. It is not in very large supply and is chiefly in use in America and as a component of the $6: 2: 2$ mixture.

It is a rather thick (glycerine-like) water-clear fluid with a faint sweetish smell. Owing to its higher viscosity it is not so liable to 'creep' and gravitate to lower levels of skin as is dimethyl phthalate. It is a paint and plastic solvent.

Its repellent properties are very high, being very similar in effectiveness and duration of effect to dimethyl phthalate. It is non-irritant and non-toxic, but may give some slight burning sensation on tender or sunburnt skin. Like dimethyl phthalate it is largely but not entirely a contact repellent, its index for repellency at a distance being $+2 \cdot 5$. It can be used for impregnating clothing which it does not stain.

It is one of a number of diols which show a high degree of repellency (see § VI).

\section{Dimethyl phthalate}

Now the most widely used modern repellent both for skin application and for impregnation of clothing, netting, etc. It is one of the most extensively used plasticizers for trade purposes and except at a time of heavy demand for military purposes was in large supply. It is easily manufactured.

Dimethyl phthalate is a water-clear, mobile oily fluid with a faint, not unpleasant smell. It has a boiling-point of $282-285^{\circ} \mathrm{C}$. It can be spread in the natural state on skin with great ease and tends rather actively to spread by 'creep' on to untreated skin at the rate of several centimetres in the hour. For this reason and due to flow by gravity along the skin sulci it tends to accumulate on dependent areas. Though to a certain extent volatile its disappearance from the skin in course of time has been considered to be chiefly due to absorption. Normally, however, loss must be largely due to wiping off by contact with clothing.

Dimethyl phthalate is non-irritant, though on the skin of the face, especially on thin skin near the eyes it causes in many individuals some slight burning sensation. Introduced into the eyes or on mucus membranes it causes severe smarting.

It is most commonly used 'straight' but can be incorporated in high percentage in various forms of creams and pastes (see $\S \mathrm{VI}$ ). It is extensively used for impregnation of clothing which it does not stain.

\section{Dibutyl phthalate}

Very similar in physical properties to dimethyl phthalate though somewhat less effective and with a higher boiling-point (b.p. $325^{\circ}$ C.). It is chiefly in use for impregnation of clothing against trombiculid mites being more resistant to washing than dimethyl phthalate. 
Other methyl group esters

In most of its physical characters and its type of repellency dimethyl phthalate is typical of many methyl, ethyl, propyl and butyl esters of phthalic acid and other aliphatic and aromatic acids where such esters have sufficient molecular weight to ensure a sufficiently high boiling-point. Many of these are as effective as dimethyl phthalate. Among such are esters of this type of citric, cinnamic, adipic and other acids.

\section{Other repellents}

Reference to substances other than the above exhibiting repellency will be found in §VI. In general it may be said that such are at most in the experimental stage as regards practical use as repellents.

It is a curious fact that of substances shown to be powerful insecticides few seem to have strong repellent properties. In addition to pyrethrum already mentioned neither derris, thanite, paradichlorobenzene nor DDT have marked repellent properties and the last-mentioned substance appears to be entirely devoid of repellent effect against mosquitoes.

\section{(2) Preparations of repellent compounds}

Besides being used in their natural state or 'straight', repellents have been very commonly embodied in lotions, creams, pastes or other preparations, either to facilitate their application or to ensure a more lasting effect. The following are the chief forms such preparations take.

\section{Lotions}

Mixtures containing the repellent dissolved in or diluted with alcohol or other thin fluid, or thickened with castor oil or arachis oil. Examples:

$\begin{array}{lrll}\text { Oil of citronella } & 4 & \text { Coumarin } & 10 \\ \text { Oil of cedar } & 2 & \text { Alcohol } & 50 \\ \text { Spirits of camphor } & 10 & \text { Glycerine } & 50\end{array}$

\section{Creams (ointment type)}

Admixtures of the repellent with some solid greasy base such as hard and soft paraffin, petroleum jelly, cetyl alcohol, lanolin, magnesium stearate with or without modifying materials. Early repellent creams were mostly of this type. Examples:

$\begin{array}{lcll}\text { Oil of citronella } & 18.5 & \text { *Dimethyl phthalate } & 70 \\ \text { Camphor } & 1 & \text { Magnesium stearate } & 30 \\ \text { Cedar oil } & 9 & \text { Dimethyl phthalate } & 80 \\ \text { Parafinum dur. } & 26.5 & \text { Cetyl alcohol } & 20 \\ \text { Petroleum jelly } & 45 & & \end{array}$

* Cream M. 8 (Porton), see § VI.
Creams (vanishing cream type)

Essentially oil in water emulsions which 'disappear' on application seeming to be absorbed by the skin, largely due to evaporation of the watery phase during manipulation. The chief requirements are, an oily or greasy base, an emulsifier such as triethanolamine, triton $\mathrm{X}$, etc. and water. Some oil in water creams are, however, of more normal cream character, e.g. in the second example given. Examples:

$\begin{array}{lrll}\text { Lanolin (hydrolyzed) } & 25 & \dagger \text { Dimethyl phthalate } & 50 \\ \text { Arachis oil } & 5 & \text { Unemul } & 40 \\ \text { Stearic acid } & 5 & \text { Prolein } & 10 \\ \text { Triethanolamine } & 2 & & \\ \text { Cetyl alcohol } & 5 & \\ \text { Oil of lavender } & 3-4 \text { drops } \\ \text { Dimethyl phthalate } & 25 & & \\ \text { Water } & 33 & & \end{array}$

Creams (waxy base type)

Mixtures of the repellent with wax and such solvent (which may be the repellent itself) as is necessary to give a correct consistence. Examples:

$\begin{array}{lrlc}\text { Rutger's 612 } & 12 & \text { †White wax } & 9 \\ \text { Beeswax } & 1 & \text { Arachis oil } & 27 \cdot 5 \\ & & \text { Dimethyl phthalate } 12 \cdot 5\end{array}$

Creams (paste type).

Mixtures of dimethyl phthalate (or other oily repellent) with powders such as zinc oxide, talcum powder, bentonite, china clay, etc. Sometimes with shellac and other components to form a permanent adherent film (see section on resistant creams). Examples:

$\begin{array}{llll}\text { Dimethyl phthalate } & 68 & \text { Dimethyl phthalate } & 26 \\ \text { China clay } & 32 & \text { Keisulgur } & 24 \\ & & \text { Magnesium stearate } \\ & & \text { Ethyl cellulose } \\ & & \text { Shellac } \\ & & \text { Other components } & 36\end{array}$

\section{Gum tragacanth preparations}

Various creams or pastes of gum tragacanth have been employed as vehicles more especially for pyrethrum. They dry leaving a thin adherent film which is not dislodged by sweating. Example:

$\begin{array}{lllr}\text { Tragacanth } & 5 & \text { Pyrethrum extract } & 0 \cdot 6 \\ \text { Alcohol } & 5 & \text { Antioxidant } & 0 \cdot 1 \\ \text { Shirlan } & 0 \cdot 1 & \text { Water to } & 100\end{array}$

Such preparations would be unsuitable for repellents of the oily compound type.

Of these preparations creams of the ointment type have frequently been noted as greasy and unpleasant in a hot climate though some, e.g. the stearate cream given as an example under this head, are cosmetically

$\dagger$ Cream S of Dr Barlow, see $\S$ VI.

$\ddagger$ Dr Hamil's cream, see $\S \mathrm{VI}$. 
excellent. The use of paraffin as a base as has been common in citronella preparations appears to have a reducing effect on repellency. Vanishing creams have not generally been found satisfactory. Owing to their 'disappearing' property, unless used in large amount, they are apt to give patchy distribution of the repellent. A waxy cream would seem to be the most effective in prolonging repellent effect and if of suitable consistence such creams spread extremely well and are pleasant to use. Lotions have not been much used.

Though it is difficult to give any wide generalization in respect to the value of different repellent preparations it would not seem that there is any very pronounced effect as regards prolongation of effectiveness by their use, and experience with dimethyl phthalate has mostly been in favour of its use straight. If it is desired, however, to use this repellent in the form of a cream either the stearate cream given (prepared under the direction of Dr G. S. Hartley, Experimental Station, Porton), or the prolein cream by Mr F. Barlow, Imperial College, South Kensington, or the white wax and arachis oil cream by Dr P. Hamil have excellent keeping qualities, are as effective as their dimethyl phthalate content and are aesthetically all excellent creams. A note on clay creams will be found later in the report.

\section{TECHNIQUE OF REARING STANDARDIZED CULTURES OF AEDES AEGYPTI}

\section{(1) Requirements}

Temperature. The most suitable temperature for rearing from egg to emergence is $28^{\circ} \mathrm{C}$. at which temperature mass development to pupae takes 6 days and completion of bulk emergence of adults a further $48 \mathrm{hr}$. For these processes it is most convenient to use one or more large incubators, e.g. height $48 \times 20 \times 20$ in. internal measurement.

For storing the adult mosquitoes after emergence for the required number of days after emergence, for making transfers and carrying out tests, as also for storing eggs for stock a temperature of $25^{\circ} \mathrm{C}$. is sufficient. This is more favourable than a higher temperature to viability of adults and quite adequate to give a good biting rate. It is also less trying to the worker who may have to spend some hours daily under such conditions. Some form of constant-temperature chamber for the purpose would seem to be essential.

Humidity. During rearing of the larvae high humidity in the incubators is unnecessary. But for the days when small cages are stacked for emergence humidity should preferably be at saturation point. This has been ensured by placing on the top of each emergence cage, of which there are eight in an incubator, one of the porous earthenware pots described below half-filled with water. Dishes of water are much less effective and if used should have a large pledget of wool placed in them.

For the constant-temperature chamber a constant relative humidity of $90 \%$ should be aimed at. The humidity should be automatically controlled with a recording dial and checked occasionally with the whirling psychrometer.* Upon the humidity largely depends freedom from mortality in the adults.

Current air. During development from egg to pupa some form of air bubbling is necessary to prevent scum formation, which, especially in the early stages and at pupation time may cause mortality or even destroy the culture. If it is not used food has to be cut down to an extent which may lead to poor size in the adult. Where current air is laid on this is readily arranged for by a tube brought through the thermometer hole in the incubator and linked up by $T$. pieces with as many bubbling tubes as are required. Where current air is not laid on some suitable device such as a small motor arranged for blowing should be used. Small motiors for the purpose used formerly to be on sale for use with aquaria (e.g. at Gamage's).

The extent of bubbling should be regulated by screw clips. About 2-4 bubbles a second should be sufficient. If too vigorous it may cause mortality at the pupal ecdysis. A T-piece with a long extension set in a tall cylinder with about 10 in. head of water interposed in the air inlet tube (Fig. 2, A) will greatly facilitate operations by maintaining a fixed pressure. Otherwise regulating one tube is liable to alter the bubbling in other tubes. It also does away with any variation in the supply pressure. It is a further great convenience if each bubbling tube is set with a piece of rubber tubing brought over the top of a large cork slit to rest on the edge of the glass vessel and fixed with two staples between which in a vertical position is the screw clip (Fig. 2, B). Tubes should be watched to see that they do not become blocked with gelatinous growth.

Breeding dishes. These should preferably be large, e.g. glass basins $30 \mathrm{~cm}$. (or even $35 \mathrm{~cm}$.) in diameter and $12-15 \mathrm{~cm}$. deep. One such vessel will accommodate 1500-2000 larvae without overcrowding. A few smaller dishes for hatching out and other miscellaneous work are desirable.

Pipettes for pupa collecting. Rubber ball teats (diameter $4 \mathrm{~cm}$.) with a 6 in. length of glass tubing (internal diameter about $6 \mathrm{~mm}$.) the edges rounded and very slightly narrowed in the flame make a convenient form of pipette for the purpose. Or a spoon may be used. For removing pupae in bulk a small sieve made by fastening a piece of mosquito

* Psychrometric tables in degrees Fahrenheit are given by Marriott (1911) and by Marvin (1941). 


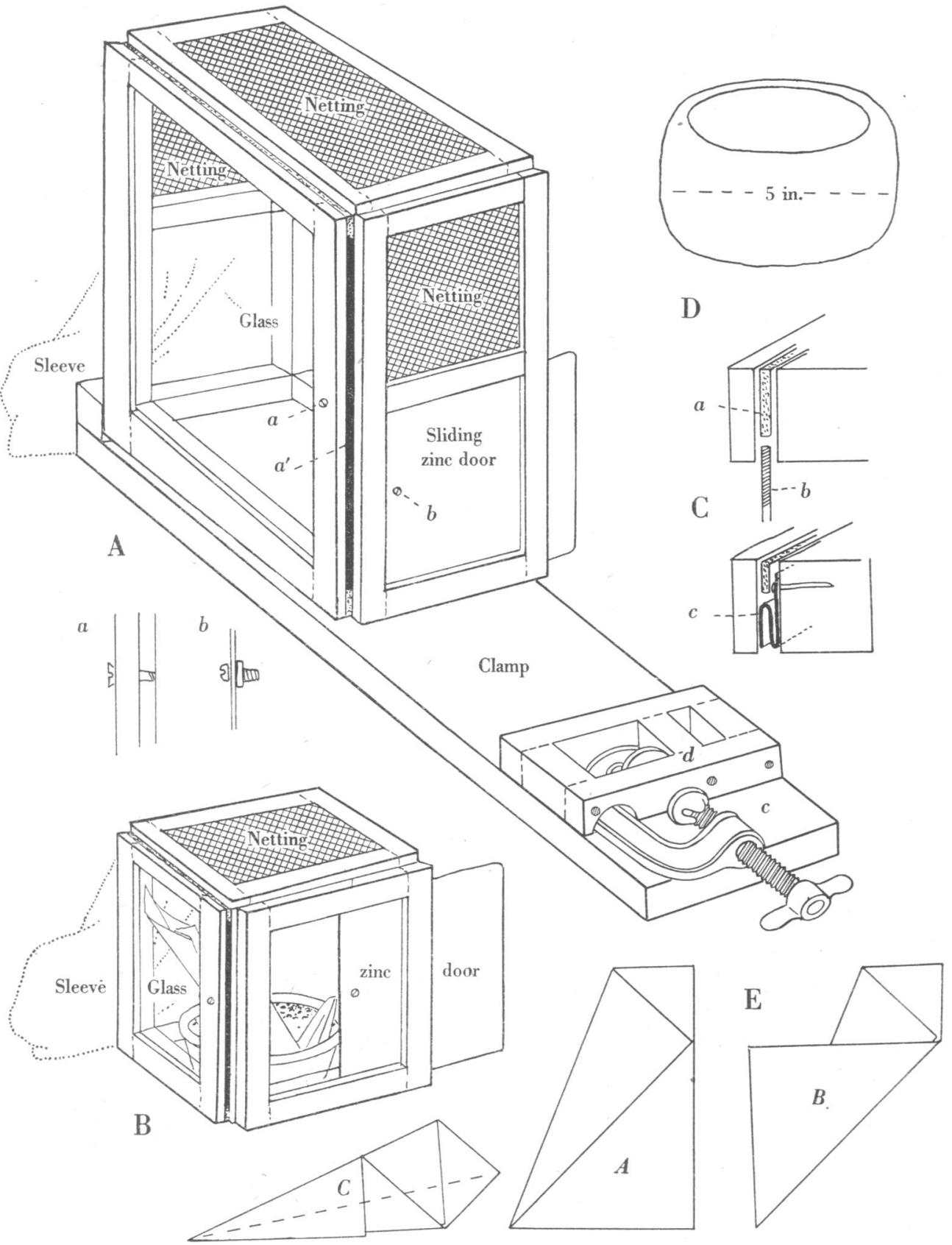

Fig. 1. A. Test cage with clamp for transferring from emergence cage. $a$, screw to prevent glass sliding out but allowing removal for cleaning or replacement; $a^{\prime}$, space through which glass sheet is removed for cleaning; $b$, small bolt and nut to prevent zinc door being pulled out from its bed during manipulations; $c$, metal screw clamp with holes drilled to enable it to be screwed down on board; $d$, loose wooden frame lying over clamp (removable). B. Emergence cage. C. Giving method of inset of glass sides and zinc door. a, cardboard strip of suitable thickness; $b$, glass sheet or zinc door; $c$, alternative method for sliding door, using folded zinc sheet runners. D. Earthenware pot as used for oviposition and other purposes. E. Method of folding filter paper fans. $A$, first fold; $B$, second fold; $C$, third, fourth and fifth folds, the last shown by an interrupted line. 
netting with a rubber band around the end of a 2 in. length of 2 in. tubing will be found very useful.

Support for breeding dishes. It is a great convenience when collecting pupae to tilt the dish so that water is brought close to the edge and so that pupae rise a little distance from the glass. A terraced block to support the dish at any desired angle saves much trouble.

Glass pots. These are such as are used for potted tongues, etc. (meat dishes), about $12 \mathrm{~cm}$. in diameter and $6 \mathrm{~cm}$. in height. They are used for counting out pupae and placing these in the emergence cages and also for other purposes. Four or five dozen may be required. cages (Fig. 1, B). They were made $8 \frac{1}{2} \times 8 \frac{1}{2} \times 8 \frac{1}{2}$ in. to allow of eight such cages with room for a humidifier pot placed on each cage being placed in an incubator of the size mentioned, viz. four above and four below the medianly placed shelf. They consisted of a frame of inch white pine scantling set on a half-inch matchboard base on which was fixed by thin slats of wood, a sleeve and on the side opposite to this a sliding zinc-sheet door, the remaining sides and the top being closed by netting, unless as is very convenient one side be glassed.

Though these cages were normally used for loading up a test cage before performing a test, they were also found convenient for holding, as a sort of unit,
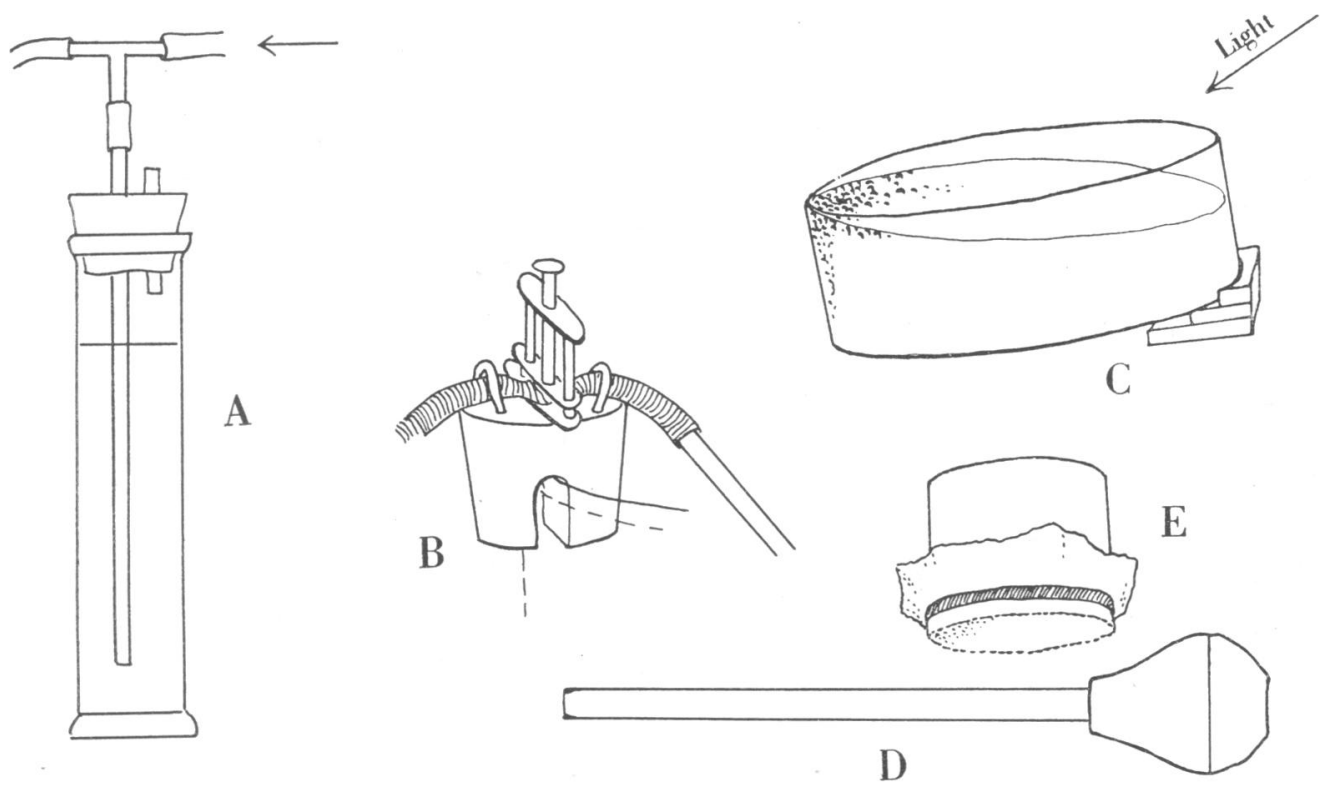

Fig. 2. A. Water-column pressure regulator interposed in air inlet tube for bubbling. B. Showing method of fixing regulating clamp for bubbling. C. Showing method of tilting breeding dish for collection of pupae. D. Rubber-ball pipette. E. Strainer for collecting bulk pupae.

Earthenware pots (egg-pots). These, said to be used as rabbit food receptacles, are invaluable. They are stout, completely unglazed red earthenware pots of about the size of the glass pots with a curved-in edge (Fig. 1, D). They form perfect pots for oviposition and the storing of eggs and as humidifiers for use with the emergence cages in the incubators. Various other forms of earthenware pots have been tried but none is so entirely satisfactory as these. They were formerly on sale from time to time at Woolworth's. Several dozen will be required.

Emergence (small or 's.c.' cages). These are designed to hold the glass pots containing pupae placed for emergence and for storing and eventual transference of batches of adults to the larger testing stock cultures of Aedes of known age, etc., for what ever purpose required. They were easily handled, easily stacked and allowed of every necessary manipulation being carried out without any handling of the mosquitoes. It is essential that the frame of the sliding door should be flush and of the same size as that for the sliding doors on the large testing cages. Each cage is clearly numbered on every side. Two dozen, twelve for each week's work, were in use.

Testing (large or L.c.) cages. These were of similar construction to the small cages but measuring $14 \times 14 \times 8 \frac{1}{2}$ in. wide, the sleeve and sliding door being at either end (Fig. 1, A). The two sides were glassed since a clear view was thus given of the con- 
tained mosquitoes whether the right or left arm was being exposed, i.e. for the control and full test. Each cage was clearly numbered on all sides. Eight such cages were in use, but more would have been convenient. On sunny days it is desirable to put them out in the sun and breeze to keep clean and sweet from any possible trace of repellent.

Stock or bulk cages. These were similar to the large cages but only 9 in. high and with one side glassed. They were found extremely useful for holding stock (residue of uncounted pupae) used for feeding for supply purposes.

Transferring clamp. This was a stout board long enough to take a large and small cage end on and fitted with a screw clamp (Fig. 1, A). It greatly assisted transferring mosquitoes to the test cages.

Filter paper fans. A convenient way of folding these is shown in Fig. 1, E. They were made by folding sheets of filter paper $2 \times 2 \mathrm{ft}$. into four and then in the other direction into three and cutting. One of these fans was always placed as a routine in every cage in which adults were being kept.

Portable electric stove. This is necessary in the winter and convenient even in summer as when placed on the table it enables a large enamel basin full of water to be kept at the desired temperature whilst collecting pupae as described later.

Killing off box. A very essential piece of apparatus. It should be solidly made without cracks to take a large testing cage allowing for a perforated zinc tray at the top. Layers of blotting paper are placed in the tray and two corked holes in the roof of the box enable the lethal agent to be poured in. The door is most conveniently made as a vertical sliding panel. As chloroform was rather expensive carbon tetra- chloride was used, the dose which would certainly kill after a given time being ascertained.

Arrangements for boiling off larvae. Besides a gas ring and a large saucepan a large sieve with bolting silk was found very useful enabling excess water to be disposed of more expeditiously than by boiling.

Food. The food for larvae was prepared from Entwistle's All-in-One Kennel Meal biscuits and bemax. The biscuits as obtainable on the market consist of broken pieces of a yellow and of a brown biscuit, the latter, probably containing dried meat or similar constituent, being in smaller amount. Since equal quantities are used the makers (Entwistle's Ltd., Vulcan Street Mills, Liverpool) have very kindly supplied these two kinds separately in $7 \mathrm{lb}$. bags and would probably so supply if told the purpose for which they are required.

The yellow and the brown biscuit and the bemax are passed several times through a mincing machine and sifted through a dredge of fine mosquito netting, the idea being to get as fine a product as is practicable. A mixture of equal parts of the three is then made and stored for use. The method of using the food is described later.

Forms. It is no exaggeration to say that after the introduction of suitably thought out forms all drudgery of keeping notes practically ceased. Not only so but previous experience had shown that without such forms there is great difficulty in reconstructing past events however carefully notes of the usual kind were kept. For rearing and up to time of test two forms only were used, each $8 \times 6 \frac{1}{2}$ in. The following are the headings, these being placed across the length of the paper in no. 1 and across the short diameter in no. 2.

(No. 1)

Egg batch .........................

Serial No. of batch

Date

\begin{tabular}{|c|c|c|c|c|c|c|c|c|c|c|c|c|c|}
\hline \multirow{2}{*}{$\begin{array}{l}\text { s.c. } \\
\text { cage }\end{array}$} & \multicolumn{3}{|c|}{ Pupae } & \multicolumn{3}{|c|}{ Pupae added } & \multicolumn{3}{|c|}{ Dead adults } & \multicolumn{2}{|c|}{ Dead pupae } & \multicolumn{2}{|c|}{ Live pupae } \\
\hline & Date & $\mathrm{F}$. & M. & Date & F. & M. & Date & F. & M. & F. & M. & F. & M. \\
\hline
\end{tabular}

Form ruled with lines for 12 entries

(No. 2)

Egg batch .........................

Serial No. of batch

Date

\begin{tabular}{|c|c|c|c|c|c|c|}
\hline \multirow{2}{*}{$\begin{array}{c}\text { Date } \\
\text { of } \\
\text { transfer }\end{array}$} & \multicolumn{2}{|c|}{ Transfer } & \multirow{2}{*}{$\begin{array}{c}\text { Serial } \\
\text { exp. } \\
\text { number }\end{array}$} & \multirow{2}{*}{$\begin{array}{l}\text { Date } \\
\text { of } \\
\text { exp. }\end{array}$} & \multirow{2}{*}{$\begin{array}{c}\text { Age of } \\
\text { mosquito }\end{array}$} & \multirow{2}{*}{ Remarks } \\
\hline & s.c. & L.C. & & & & \\
\hline
\end{tabular}

Form ruled with lines for 15 entries 
In the first form under column s.c. cage is entered the number of the emergence cage as these receive the pupae. The next three columns give the date of collection and the number of male and female pupae placed to hatch out (normally 100 of each). The column 'pupae added' was practically never used in later work as there were always ample pupae and to spare to make up the 12 cages used, but should an emergency arise presence of the column much simplifies entry. The columns to the right are available at any time it is desired to keep a record of the mortality. For the most part this was done as a routine. The nature of the egg batch number will be described later. The serial number of the batch is very useful for quickly arranging or sorting the forms.

In the second form details are given of transfers and, very important, the serial experiment number is allocated as the experiment is done. This fixes all future references to such experiment and since the numbers given in the column are serial any experiment number, however far back, can be at once picked up, and since the two forms bearing the same egg batch number and serial number are when completed clipped together, all information is at once manifest. It ought to be mentioned that in form 2 under 'Remarks' is briefly entered the name and dose of the substance tested.

\section{(2) Obtaining and storing the eggs}

For the supply of eggs mixed bulk female and male Aedes were fed on a human arm with the hand protected with a rubber glove, either in an L.c. cage into which an appropriate number of mosquitoes had been transferred from a bulk cage or in a bulk cage itself. A bulk cage, however, usually contained several thousand mosquitoes and would accommodate only two egg-pots. By placing an L.c. cage on its side four pots could be accommodated and it was usual therefore to transfer some three or four hundred mosquitoes into one or two L.c. cages. One female lays on an average 110-120 eggs. The number of eggs from feeding say 200 females would thus be at least 20,000 and as usually many more than 200 were fed in a cage, three or four pots per cage gave an ample supply. Indeed with the more heavily oviposited pots half the pot or even a quarter broken across with a hammer gave as much as was required. It was found much less trouble thus to prepare a supply for several months at one time than to make frequent small feeds. Such heavy feeding requires the subject to be largely immune to after effects of bites, but immunity is soon acquired.

Feeding was done in the constant temperature chamber at $25^{\circ} \mathrm{C}$. and was completed within $15 \mathrm{~min}$., the great majority of females having gorged by this time. The cages were put up with glass pots of water and filter fans for 2 days after which they were laid on their side and earthenware pots half filled with water substituted on the 3 rd day. It is very necessary that these pots should be rested on empty glass pots as otherwise if water seeps from them on to the floor of the cage many eggs may be laid in this situation and so wasted.

Oviposition occurs mainly on the 4th and 5th day from feeding. One or two females may sometimes oviposit even on the 3rd day. A certain number of eggs are laid on the 6th day and even later but the number is relatively small.

The egg-pots should be removed on the 7th day. If they are removed too soon there is danger of the eggs being desiccated before they are in a viable condition. When pots containing eggs were removed from the cages on the 7th day it was commonly observed that in the pipetted off water there were a few minute larvae, sometimes only one or two, sometimes twenty or so. On the 8th day the number was apt to be considerably more. Since eggs require approximately 4 days at $25^{\circ} \mathrm{C}$. to hatch, the few larvae on the 7 th day are probably from the few eggs laid on the 3rd day from feeding, those on the 8th day being some of the eggs laid on the 4th day from feeding. Not all eggs laid necessarily hatch at this time since the great majority of eggs in the pot are above the level of the water on the side of the pot and such eggs do not hatch until such time as they may be brought in actual contact with water. By removing on the 7 th day the conditions are optimum.

On removal from the cages water is pipetted off and the pots labelled in pencil with the experimental number of the cage (this is entered in the form as 'normal feed') and the date of removal, and if there is more than one pot to a cage by the respective pot number, e.g. $965 / 24$. vi. $45(2)$. This labelling is very convenient when selecting pots for hatching out and for transcribing the egg batch number on to the form.

The pots still wet are now piled one on the other, the bottom one resting on an inverted flower-pot in a dish of water, and kept until required in the constant-temperature chamber. Within a day or two they are quite dry. The water in the dish is not for humidity purposes but to protect from psoci which are apt to gain entrance and breed in the pots.

Exactly how long eggs remain viable under these conditions has not been definitely established, but some pots placed to hatch after an interval of 6 months gave a negative result and in some other cases of about this time it was thought that the number hatching was smaller than it should have been. Pots, however, have been successfully hatched out at all times up to this with no failures. With eggs laid on filter paper the period has been considerably less. 
Care should be taken throughout in regard to cleanliness of egg-pots not only to prevent still viable eggs being washed into the drains but also to prevent fouling which may facilitate premature hatching.

\section{(3) Hatching out}

For this purpose an egg-pot shown by its label to be at least a week and preferably a fortnight or more old is made use of. The reason for selecting a cage of some age is to secure simultaneous hatching of the batch. With egg batches of the age noted, i.e. from a fortnight to several months, hatching is largely complete in half an hour. The great majority of the larvae therefore start development almost simultaneously.

Having selected the egg-pot it is then submerged in a small glass basin containing water at $28^{\circ} \mathrm{C}$. to which has been added a few ounces of old culture fluid kept over from the last batch after pupa collecting and a little food. The water should just about cover the egg-pot which is then placed in the incubator with a bubbling tube inserted by the side of the pot. The addition of old culture fluid is important, since, as noted by Atkin \& Bacot (1917), and as confirmed by the author, eggs placed in clean water may not hatch, or may take much longer to hatch, or may hatch in driblets instead of simultaneously and thus cause trouble later when collecting pupae.

\section{(4) Rearing to the pupa}

On the morning after the eggs have been placed to hatch the egg-pot is removed and the dish allowed to stand facing the light for a short time to verify that hatching is satisfactory, as will be shown by masses of young larvae accumulated away from the light. The contents of the dish are now tipped bodily into a larger dish with water at $28^{\circ} \mathrm{C}$. and a little more food. It is most convenient if this second dish is still of moderate size.

On the morning of the second day the larvae are distributed into the final large breeding dishes. Hereafter though the breeding dishes are looked at and receive daily addition of food no further action is necessary beyond regulating the number of larvae to prevent overcrowding. It is quite unnecessary, indeed undesirable, to change the water. Bubbling air as described is of course maintained throughout.

The most convenient way of using the food is to mix, say, four piled teaspoonfuls of the powdered food in a mortar into a paste with tap water and dilute with tap water in a large winchester. It is a good thing to give the powder a little extra rubbing up in the mortar before beginning operations. From the winchester, after shaking, is poured out into a beaker such amount as is thought should be added to a dish. By using the same dilution and the same beaker the quantity added can be regulated. About half a pint of the above dilution was usually given to a 5 l. dish to start with and somewhat less added daily. The culture should be fairly opaque so that the larvae are only visable when they come to the surface or near the glass. There should be some sediment. At first the fluid tends to clear somewhat on standing, but later becomes permanently opaque. The fluid should not be changed. It usually smells somewhat towards the later stages, but there should be no excessive fermentation or scum. If this happens the amount of food added has been excessive or the bubbling is too slow. If too little food is added for the number of larvae present the culture medium will overnight become dark and transparent, the larvae being visable in its midst.

It is a good plan to keep a made up winchester from 24 to $38 \mathrm{hr}$. at room temperature before use, though this is not necessary, but it should not be kept longer as mould in the cultures is then encouraged. It is also desirable from time to time to clean the winchester with acid as it tends to become coated with mould which is difficult to remove.

It is important at some early stage to regulate the number of larvae and remove excess. This is most conveniently done when distributing the larvae into the large dishes on the 3rd day when the larvae are of a reasonable size to judge of their numbers. Attempts to make an enumeration of larvae beyond a very rough estimate have not led to any satisfactory method of doing this. It has been found in practice best to adopt the following method. The large dishes are laid out with about $\frac{1}{2}$ in. depth of water. Beakerfuls of the mass culture are poured into each, the larvae for a moment or two being fairly uniformly distributed. Whilst still so distributed an estimate is made of the number in a square inch. If this number is about ten no more need be added. In a dish of $30 \mathrm{~cm}$. diameter this is about 1200 larvae. As the larvae increase in size it may be found necessary still further to reduce their number. This is best done by noting the size of the moving column of larvae as these accumulate away from the light when the dishes are placed facing a window. Only experience will show what density of larvae can be safely reared in a dish without interfering with optimum development.

Overcrowding is not only liable to give smaller adults, and so introduce an element adverse to standardization, but it also adds to difficulties in the collection of pupae, for not only does too dense a swarm of pupae make operations more tedious, but lack of contrast in selection of male and female pupae greatly adds to this.

The ecdyses are passed through very rapidly, the duration of the first three larval stages being each about $24 \mathrm{hr}$., that of the fourth stage being from 2 to 3 days. The different stages are most readily determined by the diameter and colour of the larval head. 
The head diameter for any given stage is relatively unvaried. Immediately after ecdysis the head is transparent growing darker as the age in the stage increases.

\section{(5) Collection of pupae}

The first pupae, usually males, are seen on the morning of the 5th day. By the morning of the 6th day the majority have pupated. There are always, however, some, and often many, larvae still left. What is desirable is to have cultures which at collection time have a minimum of larvae present as these are troublesome if numerous when collecting the pupae. For this reason eggs were usually put to hatch early in the day.

On the morning of the 6th day the breeding dishes are removed as required from the incubator, arranged facing a window on some sheets of white paper and tilted by means of a support so that the water is brought close to the edge opposite the collector and the pupae rise to the surface not too close to the side of the basin (Fig. 2, C). The required number of female and male pupae are then pipetted out into the small glass pots half filled with water at $28^{\circ} \mathrm{C}$. for placing in the emergence cages. Before doing so, however, the turbid water in the pot is poured off and replaced until clear by fresh clean water at $28^{\circ} \mathrm{C}$. If this is not done there may be some mortality during emergence from scum formation.

All manipulations are favoured by a good light (window) facing the operator, by suitable use of white and dark background, suitable sloping of the glass pot bottom when changing the soiled water and other ways of making use of the animal's reactions. Thus negative phototaxis is overpoweringly displayed. A sloping bottom causes pupae, other things being equal, to move towards the lower end of the slope. Both larvae and pupae are very sensitive to slight jolts, but the larvae are more so than the pupae and can on occasions be got out of the way by regulating the intensity of a slight tap on the sides of the vessel.

The male and female pupae are readily distinguished at a glance on account of the great difference in size and even to some extent in their appearance and movements. No difficulty whatever should be experienced in picking out the sexes if the culture is developed optimally. If advantage is not taken at this stage to take a known number of females it is impossible to say later in a random sample either of pupae or adults what the proportion of the sexes may be. In transferring adults, for example, males may more readily pass out under some conditions than females giving a preponderance of this sex. Even the whole mass of pupae in a culture cannot be assumed to have an equality in number of the sexes.
Males were included in our test cages merely to have a fixed standard procedure and because the presence of males accentuates, by their active to and fro flight and shrill hum, the appearance of activity when the females are actively feeding.

As the pots receive their quota of pupae they are placed in emergence cages in the incubator, each cage with a porous earthenware pot half filled with water placed above it.

Throughout the operation of collecting the pupae equable conditions of temperature have been maintained by means of an electric stove on the table and a large basin of tap water kept at $28^{\circ} \mathrm{C}$. by being placed at a suitable distance from the stove by the side of the operator.

When all emergence cages have been completed the whole remaining bulk of pupae was strained off and placed to hatch out in a bulk cage. If not required these adults were killed off, but they were, if required, always available for feeding for stock or an emergency.

\section{(6) Emergence}

The completed series of emergence cages with their humidity pots are left in the incubator for $48 \mathrm{hr}$. and if emergence is then complete they are removed to the constant-temperature chamber at $25^{\circ} \mathrm{C}$. Sometimes a few pupae at this time are still not emerged. Unless, however, some larvae have been included when counting out the pupae all will have pupated within at most a few more hours.

When removed to the constant-temperature chamber a filter paper fan is inserted into each pot to give maximum humidity. The cages are then left undisturbed until they are ready on the 5th to 7 th day from emergence for use in testing, this period having been found to be that in which biting potential was well developed and mortality had not begun. It is not necessary, or desirable, to provide any food. During this period and especially about the 3rd or 4th day the mosquitoes are very alert and give rise to a shrill humming sound (largely due to the males) when disturbed by a passing shadow, etc. Copulation is also very active.

\section{(7) Transferring to the test cage}

To ensure uniform conditions and increase biting potential, mosquitoes before being used in a test were $1 \mathrm{hr}$. previously transferred to the test cage and left without water and in the light (electric).

For transferring the mosquitoes from an emergence cage to a test cage both cages were clamped door to door in the clamping device. A piece of black cloth was laid over the far end of the test cage, the doors opened and the dish and filter fan of both cages, the former still containing the shed pupal 
skins, removed. A second piece of black cloth about $2 \mathrm{ft}$. square stretched between the two hands was now vigorously flapped down several times over the emergence cage, one hand pushed with the sleeve into the cage as a final precaution and the door of the test cage quickly closed. The flapping of the cloth causes the mosquitoes to take to the wing and at the same time causes a puff of air, as can be seen by looking at the netting on the top of the test cage, carrying them into the cage. The black cloth on the far end of the cage helps to prevent them returning. The whole process takes only a minute or so, the method being found more effective and quicker than troubling to arrange the cages in relation to light, etc.

When transfer has been completed the dish with the pupal skins is examined and entries made regarding dead adults, pupae, etc. On the 5th day as a rule there were a few dead or weak males, and perhaps a dead pupa or even a dead female. By the 7th day there may be some considerable male mortality, perhaps ten to twenty and possibly one or two dead females. Mortality was considerably greater when humidifying pots were not used in the incubator during emergence.

After expiry of the hour without water the test cage is ready for the test. The technique of testing is dealt with in the next section.

\section{TECHNIQUE OF LABORATORY TESTING FOR REPELLENCY}

\section{(1) Requirements}

Temperature and humidity. All tests have been carried out in a constant-temperature room at $25^{\circ} \mathrm{C}$. and $80-90 \%$ humidity. Such a room should have the space necessary for storing the number of emergence cages used, and allowing for manipulation of cages in transferring, carrying out of tests, storing of eggs, etc.

Test cages and mosquitoes. Arrangements for these have been given in the previous section.

Arrangements for measuring dosage. For liquids the quantity suitable for application to the forearm (dorsal surface only) varies (taking such an area as 30 sq.in.) from $0.6 \mathrm{ml}$. (20 cu.mm./sq.in.) to $0.15 \mathrm{ml}$. (5 cu.mm./sq.in.). Quantities under the latter amount must usually be applied diluted in a solvent as most substances cannot be evenly spread, even with the aid of rubbing, in less amount. For such operations small pipettes holding 200, 100 and 50 cu.mm., one or two ordinary $5 \mathrm{ml}$. graduated pipettes and one or two small weighing bottles with the weight known will be found very convenient.

Solids (other than creams and pastes) must usually be applied dissolved in a solvent. The best general solvent for this purpose was found to be $n$-butyl alcohol which allows time before evaporating for the mixture to be evenly spread and which is not so slow in evaporating that it will not have disappeared from the arm by the time for testing. If not satisfactorily soluble in butyl alcohol alone a little chloroform added may do what is required. The solvent must be capable of dissolving the repellent very freely in order to get the required amount in the necessary small volume.

Creams and pastes are best measured by volume, the relation of volume to weight being determined if necessary. For this purpose a very useful device may be made from a short length (about $3 \mathrm{in}$.) of graduated $5 \mathrm{ml}$. pipette fitted with a glass plunger. The lower end should be cut off at a mark indicating a whole ml. As it is difficult to fill such a narrow tube, a filler is made of glass tubing with wide enough bore just to fit over the end of the graduated piece and fitted with a plunger. The filler packed with a sufficiency of cream with the aid of a small spatula is now placed against the end of and slightly overlapping the graduated piece and the cream pushed into this. Any measured amount can then very accurately be extruded as a worm of cream or paste.

Metal arm shield. After preliminary trials it was found preferable not to smear and expose the whole forearm, but to use the upper surface only, the under surface being protected by a metal shield. This not only prevents contamination of the sleeve and even the floor of the test cage but also gives an area which is under easy observation during the test. The shield was made of zine sheet cut and bent to fit the arm from wrist to elbow with tags cut facing forwards and backwards at wrist and elbow respectively into which, when the shield is applied, zinc bands are slipped (see Fig. 3).

Rubber gloves: These should be of loose fit and used with a little French chalk. They should be carefully protected, as described under 'Bandages' from repellent applied on the skin. Otherwise they will quickly perish.

Bandages. It is absolutely essential to exclude all possibility of mosquitoes biting any area, however small, other than the prepared area. For this purpose a bandage is applied at wrist and elbow. Suitable lengths are made if a $6 \mathrm{yd}$. bandage $(3 \mathrm{in}$.) is folded into four lengths, two of these about a foot longer than the others. These lengths are cut and about a foot slit at one end for tying. At the other end when the bandage is rolled a nick is made with the scissors which can be slipped over a tag on the shield, thus facilitating the application of the bandage by the operator. The longer bandages for convenience are marked at the nicked end with a pencil cross.

Forms. In addition to forms nos. 1 and 2 as described in $\S I V$, the second of which is used to record the experiment number, age and cage from which derived, a form on which to record results of the test as this is in progress greatly saves labour 
and the keeping of complete records in a convenient set out. For this slips $5 \times 8$ in. were used as shown below, the headings being across the short diameter of the paper. One form is used for each exposure.

\section{(2) Preliminary observations}

Before proceeding to the actual test for repellency against mosquitoes it is desirable to

Subject*

$\begin{array}{lll}\text { Substance } & & \text { Date } \\ \text { Cage } & \begin{array}{l}\text { Amount } \\ \text { Applied }\end{array} & \text { Hours } \\ \text { Age† } & \text { Exposed } & \end{array}$

\begin{tabular}{|c|c|c|c|c|c|c|c|c|}
\hline & $15^{\prime \prime}$ & $30^{\prime \prime}$ & $1^{\prime}$ & $2^{\prime}$ & $5^{\prime}$ & $10^{\prime}$ & $15^{\prime}$ & \\
\hline Control & & & & & & & & Wheals \\
\hline Settled (area) & & & & & 1 & & & \\
\hline Settled (margin) & & & & & & & & \\
\hline
\end{tabular}

* I.e. subject A, B or C according to the operator.

$\dagger$ I.e. age of the mosquitoes in days from emergence.
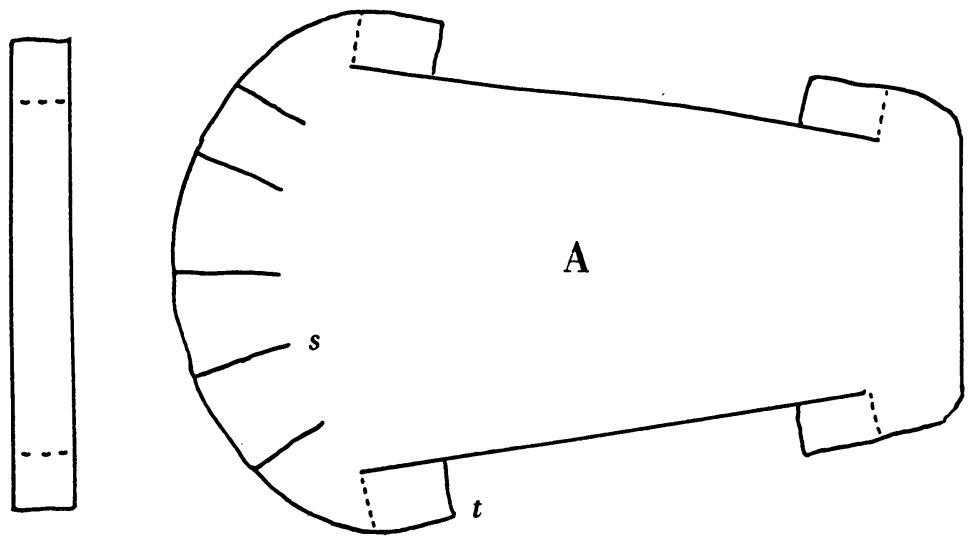

B

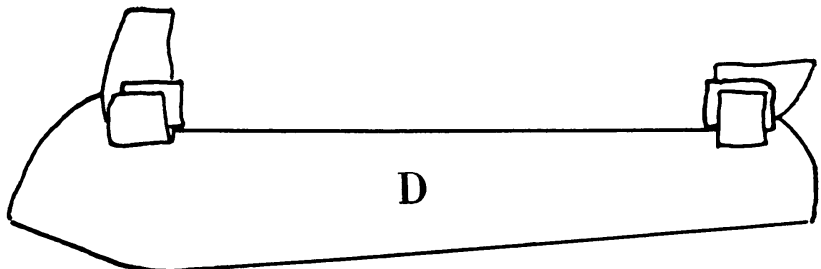

Fig. 3. A. Zinc sheet cut for folding into arm shield. Dotted lines indicate tags bent back. $t$, tag; $s$, slits to enable shield to be moulded to elbow. B. Zinc strip with ends folded to form band below elbow. C. The same at wrist. D. Shield assembled on arm.

On such forms were entered the result of the 'control', a record of settlings and then on the back at the termination of the experiment a rough sketch showing distribution of the resulting wheals, if any. record (1) the appearance and physical characters of the substance to be tested, e.g. liquid or solid, and in the case of a liquid, whether waterclear or coloured, oily or otherwise, ease with which it is spread, (2) smell, (3) boiling-point, 
or melting-point, if such be given or determined.

It was found best to describe ease of spread as the minimum quantity that, after being distributed over the area in drops or pledgets, could be spread by rubbing with the finger tip uniformly over the area. With most substances and an area of $30 \mathrm{sq}$.in. this lay between $0 \cdot 3$ and $0 \cdot 15 \mathrm{ml}$.

For recording smell the following sequence was used: strong or otherwise; pleasant or unpleasant; resembling any well-known odour, e.g. fruity, ethereal, aromatic, mentholic, etc., or more specifically where resemblance was quite definite. For degrees of intensity the following scale was found helpful: perceptible on entering room, very strong; smelt near person after application, strong; smelt easily on arm, moderately strong; smelt on arm but not obvious, faint; doubtful or absent.

A quality that can be observed roughly whilst carrying the preparations for the test is 'creep'. This can be done by marking the edge of the treated area with an ink line on some decided-upon part of the arm and taking an impression after an interval on a thin strip of white paper pressed across this. Presence of small oily spots on the paper indicates the distance creep has extended (for description of 'creep' and its relation to viscosity and surface tension, see $\S$ VIII). For information of the boilingpoint in relation to repellency see also § VIII.

During performance of the tests permanence of the film as shown by eye or retention of smell is most conveniently recorded on form no. 3 before starting the test at 2,4 or $6 \mathrm{hr}$. respectively.

In the case of solids the appearance of the film left after evaporation of the solvent is noted, e.g. whether opaque, adherent, powdery, etc. or, as is often the case where the melting-point is near that of body temperature, whether it remains oily.

In the case of creams or pastes the weight of $1.0 \mathrm{ml}$. is a useful datum to determine.

\section{(3) Preparing the arm}

Using the shield as a guide the area to be exposed is marked out on the left arm with pen and ink. The reason for choice of the left arm is that the right hand when performing the test will be available for recording. Ink is better than grease pencil as with oily repellents it is less likely to be rubbed off. The substance to be tested is now distributed in drops over the demarcated area and subsequently evenly spread with the tip of the finger, or if preferred with a glass rod. After such application an equal amount of the substance is applied in a band $\frac{3}{4}$ in. wide all round the margin of the area taking care that the ink line is covered. It is also advisable to give the inner corner by the elbow of the area itself some additional treatment. The reason for this procedure is to minimize biting on the margins of the treated area.
The corner near the elbow is very liable to loss of repellent by rubbing, bending of the arm, etc. and it is also very sensitive to bites. Though there may be some objection to such action experience has shown that it tends to minimize trouble from ambiguous results. Thus the area may remain free from any settlings throughout the period of test except in the angle referred to where there may develop one or more wheals. Such wheals obviously are less significant than those well on the area. The amount applied in the surrounding band will be found, on calculating the area, to be not very different from that originally applied to the treated area (see also under 'Assessment of results').

From such time as the repellent has been applied every precaution should be taken to prevent contact of the area with clothing or other objects. Usually there is considerable seepage of the repellent towards the lower parts of the arm and especially to under the wrist. Much of this is inevitably rubbed off, whether a shield be used or not.

\section{(4) Prepa ring the test cage}

One hour before a test is due to be performed the contents of a small cage are transferred as previously described (p. 192) to a test cage. The test cage is then left without water and exposed to the light until the test is performed.

\section{(5) Performing the 'control'}

The right arm, i.e. the untreated arm, is put up in the metal shield and with a rubber glove over the hand (bandaging is not necessary). The arm is now quickly introduced into the test cage and exposed for 15 sec. by the stop watch. It was found best to allow say 12 sec. before commencing to count and then rapidly to count the mosquitoes settled from the elbow downwards. Using 100 females the number so settling usually lay between ten and twenty.

It is essential in doing such controls that the arm should not be cold. In fact the number settling was thought to be dependent more than anything else on the degree to which, especially in cold weather, the arm had been allowed to warm up. For this reason some $15 \mathrm{~min}$. was always spent by the observer in the warm constant-temperature room before performing the control.

Absolute accuracy in such counts is scarcely possible, but with some practice a near approach can be made.

Though termed a control the procedure is more in the nature of a check indicating that the cage as regards biting potential is up to standard. It should be noted, however, that as all cages on any one day are from the same batch of larvae and treated alike, a control in the strict sense is not needed. Indeed a control carried out by exposing the normal arm for $15 \mathrm{~min}$. could only be carried out on another cage as 
practically all the females would by then be gorged and if another cage was used it would serve no useful purpose as experience had shown that over $90 \%$ of females in the cage would have gorged.

A determination of the biting potential as described was done in every case and afforded some interesting information as will be evident in the statistical study of the results given as an appendix.

\section{(6) Performing the test}

A test was normally carried out for each dosage of a substance at 2,4 and $6 \mathrm{hr}$., a fresh cage being always used for each exposure. In special cases where a more prolonged period was being tried out tests were made at 3,6 and $9 \mathrm{hr}$. During the whole period the treated arm was carefully protected from contact with clothing.

The test cage having been put up for an hour as described and the 'control' performed, the left forearm (treated arm) was placed in the metal shield, the hand protected with a rubber glove and all possible biting other than on the treated area excluded by bandaging at wrist and elbow. The arm was then introduced into the test cage and exposed for $15 \mathrm{~min}$., the number of settlings and other particulars being recorded on the form. For such record convenient symbols were used, e.g. a tick indicated a touch, felt but not usually seen, a stroke a mosquito which remained on the arm. For periods under 10 sec. the number of seconds the mosquito remained on the arm was entered by the stroke. Where it was not possible to keep count, e.g. where mosquitoes were settling and leaving in numbers an appropriate general entry was made. At the end of the exposure and allowing time for wheals to develop these were roughly mapped on the back of the form and the number on the 'margin' and on the 'area' worked out from this and entered. This map of wheals left was found to be a most useful record for future reference as it often told at a glance what had happened at the test.

Where there was obviously no repellent effect and the mosquitoes settled to feed as on the normal arm, the test was terminated at 1 or 2 min. as the case might be. The behaviour of the mosquitoes in such cases was very characteristic. They rapidly began to settle on the arm, very like a flock of starlings, and in most cases at once beginning to puncture and eventually to gorge where they originally settled. If only a small degree of repellency was present there was always hesitancy and many short alightings. In this case the test was always carried through as otherwise there was no record of punctures to make an assessment.

\section{(7) Assessment of results}

Whilst the number of settlings and the behaviour of the mosquitoes were useful in forming a general opinion of the degree and nature of repellency, it was the resulting wheals and their distribution which gave the most concrete and objective basis for assessing the degree of repellency.

Wheals, depending on their location, were obviously not all of equal significance. An entire absence of wheals showing that no mosquito had settled sufficiently long to puncture the skin was signified by the symbol +++ . Usually in such a case there were no settlings or at most one or two temporary ones. Though the occurrence of some settlings could be taken into account in a general way it was found better in assigning a fixed value for the repellency to rely on the objective fact that there were no wheals.

When one or two wheals only were present these were almost invariably on the edge of the treated area or in the inner angle by the elbow. In the former case they were commonly the result of mosquitoes resting on the metal shield and inserting the proboscis over the edge of this. In the latter case as previously noted the area is very liable to be rubbed and in any case seems to have a special attractiveness. Where some wheals but less than five wheals altogether were present the result was given as ++ . For less perfect degrees of protection a line was roughly drawn on the map of wheals separating what were considered wheals 'on the area' from those 'on the margin'. The latter were as a result of experience not restricted to wheals that had been caused by mosquitoes biting over the edge of the shield but included any within half an inch of the margin. Though this may seem rather arbitrary there were reasons for adopting the method. Thus it was clear that due to creep and the taking up of the repellent by contact with the shield the edges within such a distance were liable to be much more vulnerable than the more central parts of the area. On this basis the following assessments were made. If over five wheals were present on the area + ; if over ten on the area \pm ; if some repellency - ; if no repellency and mosquitoes settled to feed 0 .

The final result of the testing of a given dosage of a substance gave then three values, viz. those at 2 , 4 and $6 \mathrm{hr}$. respectively. Such a valuation showed not only the degree of repellency after a short time but also the extent to which the efficacy had fallen off after longer intervals. Many substances, e.g. many essential oils and more volatile compounds, gave high efficiency for the 2 or $4 \mathrm{hr}$. reading with a sudden falling off at the later readings. On the other hand some substances gave moderate results more or less constant throughout all the three tests.

When the results were ascertained, not only for some one dosage but a number of decreasing amounts of the substance, a very close comparison could be arrived at as to the efficacy of different compounds. 
A very helpful means of comparing effectiveness of two rival compounds was the ascertaining of the dosage at which three readings of +++ were consistently given. Thus repeated trials as between DMP and Rutger's 612 showed that as regards effectiveness under protected conditions there was very little to choose.

Since there are always two conditions, viz. essential effectiveness and duration of effectiveness, involved, a degree-time reading as above has the advantage of giving information about both these characters. If, however, a single figure is desired it is easy to arrange for such by giving a value to each degree of effectiveness in the triple test. Thus with a dosage of $5 \mathrm{cu} . \mathrm{mm} . / \mathrm{sq}$. in. dimethyl phthalate would be indicated by the figure 9 (nine plus signs), whereas citronellal would perhaps be five. Obviously the fuller statement gives more information.

Even used as a method of 'screening' the method here described is no more consuming of time and labour than that of time to first bite. Thus if with a dosage of $10 \mathrm{cu} . \mathrm{mm}$./sq.in. (the dosage as previously noted used at the first trial) a substance at $2 \mathrm{hr}$. does not give a satisfactory result, it is obvious that it is of no value as a repellent and it is unnecessary to proceed further.

It is not claimed that the method is that best adapted for assessing results in field tests, but even here contrast of biting rate on treated and untreated areas would seem more informative than time to first bite.

\section{COMPOUNDS AND THEIR PRE- PARATIONS TESTED BY THE UNIT}

\section{(1) Compounds}

In Table 1 is given a list of compounds that have been tested by the Unit. Opposite the name of each compound is given the boiling-point, or in the case of a solid the melting-point, the latter in brackets. To allow of ready comparison boiling-points are given, computed if necessary (see $\S$ VIII), for a pressure of $760 \mathrm{~mm}$. mercury.

In the succeeding columns are given figures indicating the results of the tests, the first four columns relating to a dosage of $10 \mathrm{cu} . \mathrm{mm}$./sq.in., the next four to one of $5 \mathrm{cu} . \mathrm{mm}$. and the third to a dosage of 2.5 cu.mm., in the special cases where such a test has been carried out.

Each of the four subordinate columns under these heads has a figure recording the results of tests at 2 , 4 and $6 \mathrm{hr}$., and, where this has been carried out, in the fourth column the result of a $9 \mathrm{hr}$. test. The figures are here used in place of the system of crosses employed in the reports and commentaries sent in by the Unit and as given in the section describing method of assessment, i.e. 3, 2, 1 and + replace
,,++++++ and + or - , whilst 0 indicates no repellency at all.

In the final column an attempt is made at a condensed valuation of the repellent properties of the substance. This is done by using the capital letters $\mathrm{A}, \mathrm{B}, \mathrm{C}, \mathrm{D}$ and $\mathrm{O}$ to represent the intensity of $\mathrm{re}$ pellency. These capital letters have respectively the same significance as the figures $3,2,1,-$ and 0 as relating to the effect at $2 \mathrm{hr}$. with a dosage of 10 cu.mm./sq.in., i.e. the first reading in the columns. Where a substance exhibits complete repellency at $2 \mathrm{hr}$. with the smaller dose of 5 cu.mm. this is shown by $\mathbf{A}^{\prime}$ and one showing complete repellency at $2 \mathrm{hr}$. with a dose of 2.5 cu.mm. by $A^{\prime \prime}$. The figures are the hours up to which a result equivalent to the initial $2 \mathrm{hr}$. effect with $10 \mathrm{cu} . \mathrm{mm}$./sq.in. is given.

Repellency so expressed is termed the rating. In practice interpretation of the rating works out in the following general way. Thus in the rating $\mathrm{A}^{\prime \prime} / 6$ : by A one knows that it is a first-class effective repellent for at least $2 \mathrm{hr}$. from application and the figure 6 shows that it has lasting powers of this effectiveness up to $6 \mathrm{hr}$. Had the initial letter been $A^{\prime}$ it would have indicated that the substance was effective for $2 \mathrm{hr}$. with a dosage of $5 \mathrm{cu} . \mathrm{mm}$./sq.in. Had the first letter been $\mathrm{B}$, it would have indicated a somewhat indifferent repellent, though as the figure 6 shows it maintained such efficiency as it had well. Other ratings are interpreted on a similar basis. The method is not perfect and for more complete information the previous columns giving the actual results must be scrutinized.

In assessing the results set out on pp. 23-5 certain tentative conclusions can be drawn as to the association of repellency with certain chemical groups. The following groups may be specially noted:

(a) Unsaturated long-chain alcohols, aldehydes, etc. and phenolic compounds such as are characteristic of many essential oils.

(b) High boiling-point esters of the lower methyl series with various high molecular weight acids, such as phthalic, adipic, cinnamic, citric acid, etc.

(c) High boiling long-chain alcohols and especially diols.

What particular components in $(a)$ are related to possession of repellent properties is not evident. It may, however, be noted that these compounds are particularly associated with citrous or other smells. In (b) the operative group appear to be the radicles $\mathrm{CH}_{3}$, and others of the methyl series as far up as the butyl radicle in ester form, whilst in $(c)$ it is apparently the position of the $\mathrm{OH}$ groups which tells, the methyl series radicles being little concerned since some diols without such groups are strongly repellent.

The negative results given by compounds with boiling-points below $250^{\circ} \mathrm{C}$. is not, it is thought, a 
Table 1

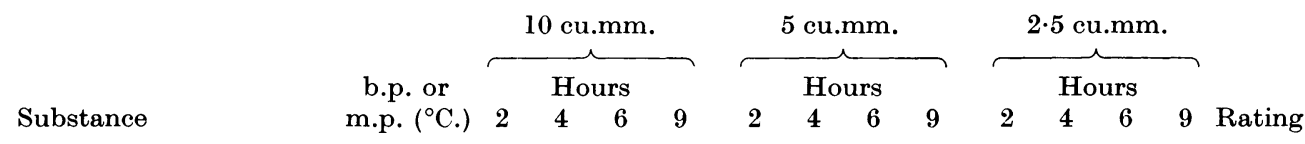

Unsaturated compounds of essential oil type:

Oil of citronella (Java)

Citronellal

Citronellol

Vetiver oil

Oil of Cassia

German prep. (Ess. oil)

Mosquitess (ol. pine)

Oil of eucalyptus

Hydroxycitronellal

Geranyl ethynyl carbinol

Eugenol and isoeugenol compounds:

Eugenol glycol ether

$p$-Chloroethyl ether of eugenol

Cis-isoeugenol

Trans-isoeugenol

Isopropyl cis-isoeugenol

Isopropyl trans-isoeugenol

$n$-Amyl cis-isoeugenol

$n$-Amyl trans-isoeugenol

Allyl cis-eugenol

Allyl trans-isoeugenol

Cis-acetyl isoeugenol

Trans-acetyl isoeugenol

Cis-isoeugenol glycol ether

Trans-isoeugenol glycol ether

Cis-benzyl isoeugenol

Trans-benzyl isoeugenol

Pyrethrins: $\dagger$

$40 \%$ extract:

$10 \mathrm{mg}$. pyrs./sq.in.

$2 \mathrm{mg}$. pyrs./sq.in.

$1 \mathrm{mg}$. pyrs./sq.in.

$0.5 \mathrm{mg}$. pyrs./sq.in.

Purified extract: Pyr. I 17·4, Pyr. II 2.3

$5 \mathrm{mg}$. pyrs./sq.in.

$2 \mathrm{mg}$. pyrs./sq.in.

$1 \mathrm{mg}$. pyrs./sq.in.

$0.5 \mathrm{mg}$. pyrs./sq.in.

$0.1 \mathrm{mg}$. pyrs./sq.in.

Pyr. I 17.0, Pyr. II 5·0:

$1.0 \mathrm{mg}$. pyrs./sq.in.

$0.1 \mathrm{mg}$. pyrs./sq.in.

Pyr. I 20, Pyr. II 17.0:

$1.0 \mathrm{mg}$. pyrs./sq.in.

$0.1 \mathrm{mg}$. pyrs./sq.in.

Pyr. I 11·4, Pyr. II $37 \cdot 7$ :

$2.0 \mathrm{mg}$. pyrs./sq.in.

$1.0 \mathrm{mg}$. pyrs./sq.in.

$0.5 \mathrm{mg}$. pyrs./sq.in.

Miscellaneous insecticides: $\ddagger$

Derris powder

Derris powder wetted alc.

Quassia, conc. inf. B.P.

$\begin{array}{cccc}. & 3 & 1 & + \\ 80 \cdot 7 & 3 & 2 & 0 \\ . & 3 & 2 & + \\ . & 3 & 2 & + \\ 246^{*} & 3 & 2 & . \\ . & 3 & 1 & + \\ . & 2 & 1 & . \\ . & 0 & . & . \\ 313 & 3 & 3 & 3 \\ & 3 & 3 & 2\end{array}$

$\mathrm{A} / 2$

$\mathrm{A} / 4$

$\mathrm{A} / 4$

$\mathrm{A} / \mathbf{4}$

$\mathrm{A} / 4$

$\mathrm{A} / 2$

$\mathrm{B} / 2$

$\mathrm{O} / 0$

$A^{\prime} / 6$

$\mathrm{A}^{\prime} / 4$

$\mathrm{C} / 2$

$\mathrm{A} / 2$

$\mathrm{A}^{\prime} / 4$

$A^{\prime} / 4-6$

$\mathrm{D} / 0$

$\mathrm{A} / 2$

$\mathrm{C} / 2$

$\mathrm{O} / 0$

$\mathrm{A} / 2$

A $/ 4$

$\mathrm{D} / 2$

$\mathrm{O} / 0$

$\mathrm{C} / 2$

$\mathrm{O} / 0$

$\mathrm{O} / 0$

$\mathrm{O} / 0$

* B.p. of cinnamic aldehyde.

$\dagger 0.5 \mathrm{mg}$./sq.in. is about equivalent to a liberal application with 1.0 pyrethrins cream.

¥ Tested in early stage of inquiry and results not strictly comparable with other entries in the table. 
Table 1 (continued)

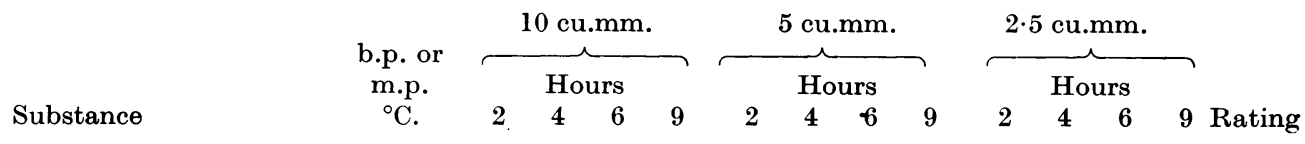

Miscellaneous insecticides (continued):

Thanite

Flit

Nicotine $2 \%$ in water of $95-98 \%$ extract

DDT $1 \%$ impreg. cloth

Saturated long-chain monohydric alcohols: $\dagger$

$58 j$

$58 k$

Unsaturated long-chain monohydric alcohols:

$46 a$

$46 c$

$46 d$

227

231

265

Saturated long-chain dihydric alcohols: $†$

2 Ethyl hexane diol-1:3 (Rutgers 612):

$46 e$

$58 c$

$58 e^{*}$

$58 f^{*}$

$58 g$

$58 h^{*}$

$58 i^{*}$

Unsaturated long-chain dihydric alcohols $†$ (diols)

$(141)^{*}$

$(142)^{*}$

$46 \mathrm{~g}$

$46 h$

$58 a$

$58 l$

Cyclic alcohols:

Methyl cyclohexanol

Ethynyl cyclohexanol

$58 b$

Aminoalcohol: 18+

Phthalates of high boiling-point alcohols :
R.D. $252 \mathrm{~A}$
R.D. $252 \mathrm{C}$
R.D. 252 D
R.D. $252 \mathrm{E}$
R.D. $252 \mathrm{~L}$
R.D. $252 \mathrm{M}$
R.D. $252 \mathrm{~N}$

Phthalate esters of the methyl series:

Dimethyl phthalate

DMP-dibutyl phthalate

Methyl ethyl phthalate

Diethyl phthalate

Methyl isopropyl phthalate

Ethyl isopropyl phthalate

Di-isopropyl phthalate

Methyl $n$-butyl phthalate

$n$-Propyl isopropyl phthalate

Dibutyl phthalate

Citronellyl methyl phthalate

Geranyl methyl phthalate

Methyl cyclohexyl methyl phthalate

328
330
333
331
338
340
$(84-91)$
$+($ diols)
331
317
.
265
314
372

$\begin{array}{lll}2 & 1 & 1 \\ 2 & . & . \\ 3 & 0 & 0 \\ 0 & . & \end{array}$

2

$\mathrm{B} / 2$

$\mathrm{B} / 2$

$\mathrm{A} / 2$

$\mathrm{O} / 0$

$\mathrm{C} / 2$

$\mathrm{D} / 2$

$\mathbf{A}^{\prime} / 4$

$\mathrm{O} / 0$

$\mathrm{C} / 2$

$A^{\prime} / 6$

A/ 6

$\mathrm{D} / 6$

$\mathrm{C} / 2$

$\mathrm{C} / 6$

$\mathrm{B} / 2$

$\mathrm{C} / 2$

$\mathrm{O} / 0$

A/2

D/2

$\mathrm{C} / 2$

B/4

$A^{\prime \prime} / 6$

B/2

$165-180$
298

$\mathrm{O} / 0$

A/2

$\mathrm{D} / 2$

$\mathrm{D} / 2$

$\mathrm{O} / 0$

$\mathrm{D} / 2$

$\mathrm{O} / 0$

$\mathrm{D} / 2$

$\mathrm{O} / 0$

$\mathrm{C} / 2$

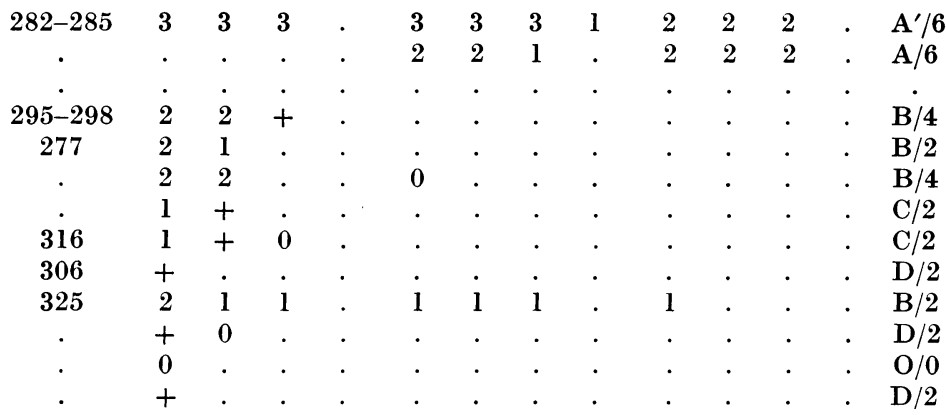

$\dagger$ An asterisk is placed after those without any substitution groups other than the OH group or groups of the alcohọl. 
Table 1 (continued)

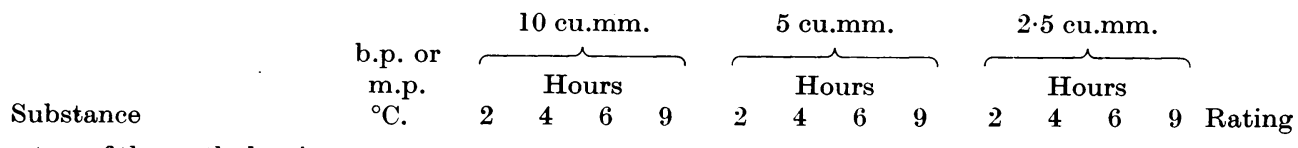

Terephthalate esters of the methyl series:

Dimethyl terephthalate

Diethyl terephthalate

Over

Di-isopropyl terephthalate

Di- $n$-butyl terephthalate

$(100)$

(55-56)

Liq.

Hydrophthalate methyl esters:

Dimethyl 4-tetrahydro-phthalate

271

210

0
0
0
+

Dimethyl hexahydro-phthalate

hyl series:

Other high boiling-point esters of the methyl seri
Diethyl adipate

Di-isopropyl adipate

Di-isobutyl adipate

Diethyl cinnamate

$n$-Propyl cinnamate

Isopropyl cinnamate

$n$-Butyl cinnamate

Isobutyl cinnamate

Trimethyl citrate

Triethyl citrate

Tri- $n$-propyl citrate

Tri-isopropyl citrate

Tributyl citrate

\section{2}

344

271-275

323

297

352

342

(76-77)

Tri-isobutyl citrate

375-380

454-462

$420-425$

Diethyl benzal malonate

Phenyl methyl maleate

348-354

Phenoxy ethyl acetate

Lower boiling-point esters (b.p. below 250) :

Ethyl lactate

Amyl butyrate

Dimethyl maleate

Methyl benzyl maleate

Ethyl benzoate

Methyl benzoate

Methyl $o$-toluate

Methyl phenyl acetate

154

Ethyl phenyl acetate

Methyl cyclohexanol

Isobutyl phenyl acetate

Allyl phenyl acetate

Methyl cyclohexanone

Methyl cyclohexanyl acetate

Ethynyl cyclohexanol

212-215

198-200

207-210

219-228

226-228

165-180

247

244

163-175

175-190

189

Sulphur compounds:

Thioxan

Thiodiglycol

M.P. 17

Diethyl xanthogen

Dimethyl thianthrene

Other compounds :

Tricresyl phosphate

Sodium dicresyl phosphate

Indalone

Trichloroacetyl aminoethyl chloride (in $15 \%$ ointment)

410

$\begin{array}{llll}410 & + & & \\ . & 3 & 1 & 1 \\ . & 3 & 2 & 2 \\ . & 3 & 3 & 3\end{array}$

$31+$

$\mathbf{A}^{\prime} / 4$

A $/ 4$

$\mathrm{A} / 2$

$\mathrm{A}^{\prime} / 2$

$\mathrm{A}^{\prime} / 6$

$\mathbf{A}^{\prime} / 6$

A $/ 4$

$\mathrm{A}^{\prime} / 6$

$\mathrm{A} / 2$

$\mathrm{A} / 2$

$\mathrm{O} / 0$

$\mathrm{C} / 2$

$\mathrm{A}^{\prime \prime} / 2$

$\mathrm{B} / 2$

$\mathrm{A} / 2$

$\mathrm{B} / 6$

$\mathrm{A} / 2$

$\mathrm{D} / 2$

$\mathrm{D} / 2$

$\mathrm{O} / 0$

$\mathrm{O} /-$

$\mathrm{O} / 0$

$\mathrm{D} / 2$

$\mathrm{O} / 0$

$\mathrm{O} / 0$

$\mathrm{O} / 0$

$\mathrm{O} / 0$

$\mathrm{O} / 0$

$\mathrm{O} / 0$

$\mathrm{A} / 2$

$\mathrm{A} / 2$

$\mathrm{O} / 0$

$\mathrm{O} / 0$

$\mathrm{O} / 0$

$\mathrm{O} / 0$

$\mathrm{O} / 0$

$\mathrm{D} / 2$

$\mathrm{A} / 2$

$\mathrm{O} / 0$

$\mathrm{D} / 2$

A/2

A/2

$A^{\prime \prime} / 6$ 
coincidence, but due to the fact that such substances have entirely volatilized before the $2 \mathrm{hr}$. period when such compounds are tested. That this should be so with compounds having boiling-points up to say $100^{\circ} \mathrm{C}$. is not unexpected. Experience, however, during testing indicates a much higher critical boiling-point, since up to $200^{\circ} \mathrm{C}$. disappearance of all trace of the film, and even smell where this is possessed by the compound, is usual and the limit where a repellent effect is given at more than $2 \mathrm{hr}$. would seem to be round about $250^{\circ} \mathrm{C}$. Most repellents giving a longer repellent period than $2 \mathrm{hr}$. have boiling-points lying between 250 and $350^{\circ} \mathrm{C}$. Over this there seems to be a tendency for reduced effectiveness, though such effectiveness as is possessed is maintained (see §VIII where the relation of boiling-point to repellency is further discussed).

The results given under the heading purified extracts of pyrethrins were obtained with desensitized extracts prepared by $\mathrm{Dr}$ Tattersfield. Probably repellency in this case is largely due to rapid lethal effect. The results, however, would seem to show that, whatever the mechanism, pyrethrin I is much more effective than pyrethrin II (see also § IX). The dosage given by a $1.0 \%$ pyrethrins preparation thickly smeared on the skin would be in the neighbourhood of $0.5 \mathrm{mg}$./sq.in., an amount which in the form of these pure extracts was but poorly effective. Since preparations containing more than $1.0 \%$ pyrethrins have rarely been considered desirable, poor results from pyrethrum creams with such amounts as $0.3 \%$ pyrethrins as noted in the second half of this section would seem to be explained.

Among negative results of interest are those with such compounds as citronellyl and geranyl phthalate, and those with the phthalates of some high boiling-point alcohols. Negative results with the terephthalate esters may be partly explained by most of these being solids at skin temperature, but that the liquid dibutyl terephthalate was so poorly effective would point to the para position being in this case antagonistic to repellency.

\section{(2) Preparations}

In Table 2 are given brief particulars of preparations tested, for the most part creams and pastes of dimethyl phthalate. The table gives the composition, a brief description of the preparation and its efficacy expressed as when dealing with compounds in the last section. In the last two columns is noted the effect on the physical characters of the preparation of storage at room temperature in a closed receptacle for the period in months noted.

So far as the creams here tested are concerned certain conclusions would appear to be justified.

The lack of effective repellency in those $0.3 \%$ pyrethrin creams tested is very obvious and as already noted in the previous section even pure extract in this amount (about $1.0 \mathrm{mg}$. pyrethrins/ cu.mm. when using $2 \mathrm{ml}$. of cream to the forearm) gives very poor repellent effect.

Of the DMP creams those with unemul and solvar, though as effective as their DMP content applied straight, are so liable to separation of the DMP that they cannot be considered satisfactory. An excellent stable cream of this type (cream S of Mr Barlow) with unemul and prolein (propylene glycol dioleate) and $50 \%$ DMP is, however, to be noted.

Another type of cream giving poor results are those making use of cellulose acetate, and cream RG-30 which possibly has this substance as a base and is cosmetically acceptable was noticeably not very effective, possibly from low DMP content.

One of the best creams, pleasant to use, effective and seemingly quite stable under ordinary conditions is the $70 \%$ DMP Porton cream M. 8, with no other materials in its composition than DMP and magnesium stearate. Another excellent cream is that with a waxy base by Dr Hamil which shows no sign of deterioration in the time it has been under observation and which is pleasant to use, spreads with great ease and is as effective as its DMP content used straight.

Of those creams with an absorbent powder base that with zinc oxide is cosmetically quite unsuitable on account of the unsightly dead white film given. In this respect white china clay offers a great contrast since the mixture of DMP and clay when smeared on the skin leaves no trace of whiteness until after many hours the DMP has been volatilized or absorbed. An account of experiments made in this connexion is given in § VII.

Those 'resistant' creams tested, which probably all depend on the presence of shellac among their constituents combined with a powder base, have been extremely difficult to spread and on this account alone are unsatisfactory. In this direction also an extensive series of trials have been made as to an improved form of cream of this type and the results recorded in $\S$ VII.

In general it may be said that as a result of many trials all creams other than those of the 'resistant' type, of which a satisfactory preparation has still to be developed, are no less liable than DMP applied straight to be removed by rubbing from clothing, etc., and therefore possess no advantage over the latter method in respect to duration of effect. But should it be merely desired to have DMP in the form of a cream, then probably the simplest and best preparations are:

(1) DMP in a waxy base (Dr Hamil's cream or variants of this to suit different climates).

(2) The 70\% magnesium stearate cream (Porton).

(3) A simple $60 \%$ DMP white china clay mixture (for further particulars see § VII). 


\section{Preparation}

$0.3 \%$ pyr. trag. cream:

G. tragacanth

extract $47 \cdot 4$

Alcohol

Shirlan

Antioxidant

Water to

\section{$5 \cdot 0$}

$0 \cdot 63$

$5 \cdot 0$

$0 \cdot 1$

$0 \cdot 1$

100

$0.3 \%$ pyr. trag. cream:

G. tragacanth

$4 \cdot 25$

Pyr. extract $47 \cdot 4 \%$

Alcohol

$0 \cdot 63$

Shirlan

$4 \cdot 25$

Antioxidant

$0 \cdot 1$

Glycerine

$0 \cdot 1$

$5 \cdot 0$

Unemul

$10 \cdot 0$

Water to

100

$0 \cdot 3 \%$ pyr. composite unemul cream:

$\begin{array}{lc}\text { Soft paraff. } & 2 \cdot 0 \\ \text { Spray base } & 2 \cdot 5 \\ \text { Pyr. extract 47.4\% } & 0.63 \\ \text { Shell oil P. 31 } & 10 \cdot 0 \\ \text { Unemul } & 52.0 \\ \text { G. tragacanth } & 0.5 \\ \text { Antioxidant } & 0.1 \\ \text { Hydroquinone } & 0.005 \\ \text { Pyrocatechol } & 0.005 \\ \text { Shirlan } & 0.1 \\ \text { Water to } & 100\end{array}$

$0.3 \%$ pyr. vanishing cream:

Stearic acid $\quad 16 \cdot 0$

Ammonia $2 \% \quad 15.0$

Sesame oil $\quad 2 \cdot 0$

Pyefly 4.6

Kerosene $\quad \mathbf{3 \cdot 4}$

Water $\quad 59 \cdot 0$

Table 2

\author{
Description \\ Yellow, soft, trans- \\ lucent, gelatinous, \\ paste. Dries \\ giving adherent \\ scaly deposit on \\ skin
}

As above but film less scaly

Putty-coloured paste with poor spread leaving little effect as with vanishing cream

$$
0 \cdot 1+\text {. Do. }
$$

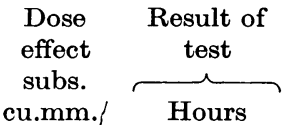

Period

Effective- under $\begin{array}{llllccc}\text { sq.in. } & 2 & 4 & 6 & \text { ness } & \text { obs. Change }\end{array}$

$0 \cdot 1+++$ Poor +26 Unchanged

$0.05+++$ Poor

-

Dimethyl phthalate unemul creams:

$\mathbf{7 4 . 5} \%$ DMP (cream K):

DMP 190

Solvar $20 / 15$ (20\% aq.) 25

Unemul

40

$76 \%$ DMP (cream H):

DMP 190

Solvar $20 / 15$ (10\% aq.) 20

Unemul

40

$76 \%$ DMP (cream $\mathrm{H}^{\prime}$ ):

As above but heated at $60^{\circ} \mathrm{C}$. for $12 \mathrm{hr}$.

$50 \%$ DMP (cream H.I.):

DMP $\quad 50$

Unemul 24

Solvar 12

Water 14
Soft, rather floppy, white cream. Spreads well leaving flecks, rubbery deposit

As above but less floppy and less residue on rubbing

As above

Soft cream, rather floppy, leaving some rubber.

like residue
$0 \cdot 1 \quad 0 \quad . \quad$. Ineffective

$0.05 \quad 0$

Ineffective

26 Somewhat

dried but

still soft

$\begin{array}{llllllll}0 \cdot 15 & 0 & . & . & \text { Do. } & 26 & \begin{array}{c}\text { Somewhat } \\ \text { dried }\end{array}\end{array}$

$25 \cdot 0$

$12 \cdot 5$

$6 \cdot 25$

3

$\begin{array}{llll}3 & 2 & 3 & \text { Less }\end{array}$

Less effect. $\quad 27$

than DMP

Some separa.

content

tion, DMP

straight

$12 \cdot 5$

3

3
As effect.
as DMP,
content

straight

$\begin{array}{llll}5 \cdot 0 & 3 & & \end{array}$ Do

27 Separation, DMP

$\begin{array}{lllll}5 \cdot 0 & 3 & 3 & 3 & \text { Do. }\end{array}$

12 Do. 
Table 2 (continued)

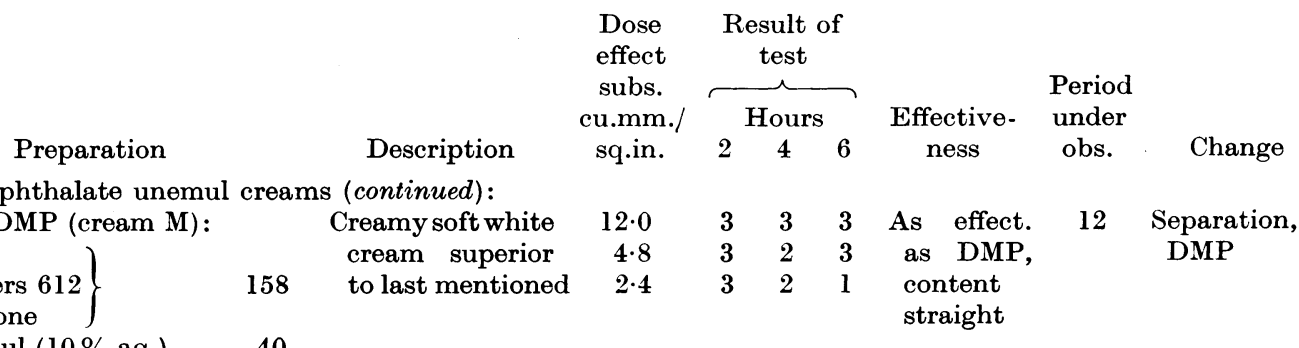

Dimethyl phthalate unemul creams (continued)

$\begin{array}{llllllll}\text { Similar to above } & 4.8 & 3 & 2 & 2 & \text { Do. } & 12 & \text { Do. }\end{array}$

$72.5 \%$ DMP (cream N): $\mathbf{7 2 . 5} \%$ DMP (cream M)

DMP

Rutgers 612
Indalone

Unemul (10\% aq.)

Solvar (10\% aq.)

DMP

Rutgers 612$\}$

158

Unemul (10\% aq.)

Solvar (10\% aq.)

$50 \%$ DMP (cream S) :

DMP $\quad 50$

Unemul 40

Prolein 10

DMP unemul with $0 \cdot 16$ pyrethrins :

Cream L:

As $\mathrm{K}$ but with 0.2 pyrethrins dissolved in DMP

Cream J :

As $\mathrm{H}$ but with 0.2 pyrethrins dissolved in DMP

DMP stearate creams :

$26.6 \%$ DMP in Porton antigas base No. 6 :

DMP 20

Mixed sodium and pot. stearate

\section{6}

Hardened ground nut oil 10

Water

39

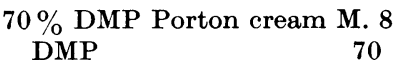

Magnesium stearate $\quad 30$

DMP cellulose acetate creams :

$50 \%$ DMP Porton cream M. 6: Somewhat trans-

$$
\text { DMP }
$$

Triacetin

Cellulose aceto-butyrate $\mathbf{3 \cdot 5}$

Magnesium stearate

20

$90 \%$ DMP jelly :

DMP 90

Cellulose acetate 5

Bentonite

lucent, gelatin-

ous and rather

sticky

An excellent

cream, soft,

creamy and

pleasant

Yellowish cream,

otherwise as

cream $\mathbf{K}$

Yellowish cream,

otherwise as

cream $\mathrm{H}$

Flaky white, poor spread, leaves

little visible layer

$\begin{array}{rrrrr}10 \cdot 0 & 3 & 2 & 2 & \text { Do. } \\ 5 \cdot 0 & 3 & 3 & 2 & \end{array}$

22 Unchanged

Greenish brown, stiff, lumpy jelly.

Unsuitable

$95 \%$ DMP jelly :

DMP 95

Cellulose acetate 5

DMP in waxy base:

$25 \%$ DMP cream (Dr Hamil):

DMP 12.5

White wax 9

Arachis oil $\quad \mathbf{2 7 \cdot 5}$

DMP or

White wax 11

Arachis oil $\quad 26 \cdot 5$
Clear, stiff jelly.

Unsuitable

Excellent cream with excellent spread and pleasant

A harder cream

\begin{tabular}{|c|c|c|c|c|c|c|}
\hline 15 & 3 & 3 & 3 & Effective as & 22 & Unchanged \\
\hline 10 & 2 & 2 & 2 & DMP, con- & & \\
\hline $4 \cdot 7$ & 3 & 3 & 3 & tent straight & & \\
\hline 10 & 2 & 2 & 1 & Rather less & - & - \\
\hline 8 & 2 & 3 & 2 & effective & & \\
\hline 5 & 2 & 1 & 2 & $\begin{array}{l}\text { than DMP, } \\
\text { content straig }\end{array}$ & & \\
\hline $6 \cdot 0$ & 3 & 2 & 3 & $\begin{array}{l}\text { Effective as } \\
\text { DMP, con- } \\
\text { tent straight }\end{array}$ & 20 & $\begin{array}{c}\text { Some separa- } \\
\text { tion of DMP }\end{array}$ \\
\hline $6 \cdot 0$ & 3 & 2 & 2 & Do. & 20 & Unchanged \\
\hline $5 \cdot 0$ & 3 & 3 & 2 & Do. & 14 & Do. \\
\hline
\end{tabular}


Table 2 (continued)

\section{Preparation}

DMP powder base creams:

$50 \%$ DMP:
DMP 50
Zinc oxide 50
$60 \%$ DMP:
DMP 60
Bentonite 40
$68 \%$ DMP (Mr Barlow)
DMP
China clay (white)
(See also § VII)

DMP resistant creams: $26 \%$ DMP. Cream R-47

(U.S.A.):

DMP

Kieselguhr

Magnesium stearate

Ethyl cellulose

Shellac

Dupenol Dupont emulsif. 0.5 and other ingredients

Cream R-32. Semi-liquid prep.: DMP content unknown Probably some shellac

Cream RG-30 (U.S.A.): DMP content unknown Cream R-33 (U.S.A.): DMP content unknown Probably some shellac

Cream R-56 (U.S.A.)

\section{Description}

Good consistence

but very unsightly

Greenish brown paste. Poor spread. Unsuitable .

Soft greyish cream with excellent spread and leaving no appearance of clay

Dark putty-coloured paste diffi. cult to spread. Dries to adherent film

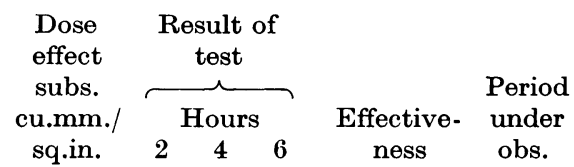

Change

\section{$\begin{array}{llllll}7 \cdot 0 & 3 & 3 & 2 & \text { Effective as } & -\end{array}$} DMP, content straight

Effective as - Unchanged tent straight

Some separation of DMP

\begin{tabular}{|c|c|c|c|c|c|c|c|}
\hline $\begin{array}{l}\text { Reddish brown } \\
\text { creamy liquid } \\
\text { sedimenting on } \\
\text { standing }\end{array}$ & $\begin{array}{l}1 \mathrm{ml} . \\
\text { cream }\end{array}$ & 1 & 1 & 1 & $\begin{array}{l}\text { Poorly } \\
\text { effective }\end{array}$ & - & Unchanged \\
\hline $\begin{array}{l}\text { Jelly drying to } \\
\text { firm film }\end{array}$ & $\begin{array}{l}1 \mathrm{ml} . \\
\text { cream }\end{array}$ & 2 & 1 & 1 & Do. & - & Do. \\
\hline $\begin{array}{l}\text { Reddish grey } \\
\text { paste very diffi- } \\
\text { cult to spread. } \\
\text { Dries to adher- } \\
\text { ent film }\end{array}$ & $\begin{array}{l}1 \mathrm{ml} . \\
\text { cream }\end{array}$ & 2 & 2 & 1 & Do. & 一. & $\begin{array}{l}\text { Tendency to } \\
\text { dry, becom. } \\
\text { ing stiffer }\end{array}$ \\
\hline $\begin{array}{l}\text { Greyish, other- } \\
\text { wise like } \mathrm{R}-33 \\
\text { cream }\end{array}$ & $\begin{array}{l}1 \cdot 5 \\
1 \cdot 0 \\
1.5\end{array}$ & $\begin{array}{l}1 \\
1\end{array}$ & $\begin{array}{l}2 \\
+\end{array}$ & $\begin{array}{l}+ \\
2\end{array}$ & Do. & - & Do. \\
\hline
\end{tabular}

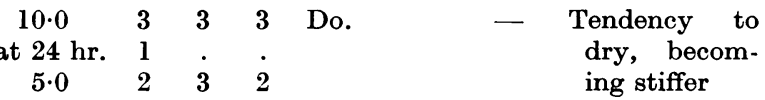

No special difficulty exists in regard to the first of these requirements and several excellent creams have been indicated in the section on 'Preparations of repellents'.

In regard to the second requirement experience has so far been that no cream of the ordinary ointment or emulsion type seriously, if at all, extends the period of protection over that given by use of DMP straight. It has been pointed out, however, that absorptive powders, notably zinc oxide when mixed with DMP, have this power. A physically excellent cream can be made by mere mixture of zinc oxide and DMP which has a high DMP percentage content loss of efficiency from rubbing of clothing and sweating. 
and which is very effective. But such a preparation is unfortunately cosmetically inacceptable.

The third desideratum is largely that which is required to ensure the second. For if the treated area is protected from rubbing by clothing or other contact the period of complete protection with DMP (assuming also absence of profuse sweating) will be 6 or even $9 \mathrm{hr}$., which is all that can be required of any repellent application. But where the subject proceeds about his business (and here must be taken into account the obvious contacts when sleeping) contact with clothing alone cuts the protection period to at most $2 \mathrm{hr}$. The possibility that some form of preparation might prevent this effect assumes great importance and is indeed at present the greatest desideratum of all in respect to repellency from application to the skin, where prolonged effect is required.

Preparations containing zinc oxide and other absorptive substances have indeed been put forward with claims so to extend the protection period. But all those that have been examined and tested in this Unit are not only unpleasant in appearance and very difficult to apply but have had a relatively low DMP content and are not sufficiently effective to counteract their obvious disadvantages. Some observations in this direction using the absorptive substance china clay as a base have been made by the Unit and though success has not been very great the results are worth recording.

\section{(2) China clay as a basis for DMP creams}

If DMP be incorporated with say $50 \mathrm{~g}$. of a good quality china clay, such as Speswhite, $20 \mathrm{ml}$. are taken up before the loose powder shows any tendency to pack on pressure. Five more ml. produce a dry greyish white granular mass which compacts somewhat on pressure. A still further $5 \mathrm{ml}$. (in all $30 \mathrm{ml}$.) are taken up before the moist granular mass compacts from slow manipulation. It is not until $40 \mathrm{ml}$. have been added that anything resembling a paste is formed.

Addition of still more DMP, say up to $80 \mathrm{ml}$., gives an excellent, easily spread cream or paste of any desired consistence, depending upon the amount of DMP added. Not only has such a cream excellent spreading qualities, but unlike the product from zinc oxide it leaves when spread on the skin no whiteness at all, the DMP making the china clay in a thin layer practically transparent. This freedom from objectionable appearance combined with excellent keeping qualities and a very high DMP percentage content not only suggested that creams of this kind might be suitable for a simple DMP cream, but also suggested the possibility that a good 'resistant' DMP cream might be made with china clay as the absorptive base.
Four china clays were available for study, a pure white clay 'Speswhite' and three grey clays (A-C). Trial was made as to the amount of DMP which these clays would take up with the following result, using in each case $10 \mathrm{~g}$. of the clay.

$\begin{array}{lcc}\text { Speswhite } & \begin{array}{c}\text { ml. required } \\ \text { to give a } \\ \text { stiff paste }\end{array} & \begin{array}{c}\text { ml. giving an } \\ \text { easily spread } \\ \text { paste }\end{array} \\ \text { Clay A } & 8 & 16 \\ \text { Clay B } & 5-6 & 9 \\ \text { Clay C } & 5-7 & 10 \\ & 5-6 & 10\end{array}$

The creams made with Speswhite were almost white, those with the other clays dark grey. Apart from this the three other clays were obviously less absorptive than Speswhite, which was therefore selected.

A mixture of $40 \mathrm{~g}$. Speswhite and $60 \mathrm{ml}$. DMP (cream 3) gave an excellent cream which spread readily leaving no appearance of white on the arm. Its DMP content $\mathrm{w} / \mathrm{w}$ was 64 and $\mathrm{v} / \mathrm{v} 75$. It will be seen from the tests in Table 3 that when the treated area was, as usual in the tests, protected from contacts this cream gave results quite comparable with those for its DMP content.

The cream, however, as will be seen from the table when subjected to 'rubbing' failed to show any obviously better results than with DMP straight.

The dose of DMP which just suffices under protected conditions to give complete protection for $6 \mathrm{hr}$. when applied straight has been found to be under the test conditions 5.0 cu.mm./sq.in. (see Table 3). The results from the same dosage subject to 'rubbing' are also shown. By employing such a dose of any preparation as contains this critical amount of DMP, the efficacy of such a preparation as compared with DMP used straight can be determined, as also whether it is more resistant to rubbing.

The method employed to simulate contact with clothing has been each $30 \mathrm{~min}$. to pass a 6 in. strip of 3 in. bandage several times firmly over the area from elbow to wrist. Attempts to arrive at a more standard method were not successful.

Since there was no reason to believe that this clay cream was more resistant to. rubbing than DMP straight and several other creams that had been tested in this way it was thought that what should be aimed at was a preparation made sufficiently soft by addition of a volatile substance to spread readily, but which when this substance evaporated would leave a firmer mixture of clay and DMP behind. For this purpose a cream was made only just capable of being compacted (cream 5: Speswhite 40, DMP 30, DMP content w/w 47, v/v 64) and to $96 \mathrm{~g}$. of this were added $12 \mathrm{ml}$. of thin nujol. This turned out to be a liquid and the film left lacked cohesion and was readily removed by rubbing. 
Table 3

\begin{tabular}{|c|c|c|c|c|c|c|c|}
\hline \multirow{3}{*}{$\begin{array}{c}\text { Paste } \\
\text { no. }\end{array}$} & \multirow{3}{*}{ Date of exp. } & \multirow{3}{*}{$\begin{array}{c}\text { DMP } \\
\text { content } \\
\%\end{array}$} & \multirow{2}{*}{$\begin{array}{c}\text { DMP } \\
\text { cu.mm./ } \\
\text { sq.in. }\end{array}$} & \multirow{2}{*}{$\begin{array}{c}\text { Age } \\
\text { mosquito }\end{array}$} & \multicolumn{3}{|c|}{ Result of test at hours } \\
\hline & & & & & 2 & 4 & 6 \\
\hline & & & \multicolumn{5}{|c|}{ DMP, straight, protected } \\
\hline- & 21. xii. 43 & - & 5 & 5 & +++ & ++ & +++ \\
\hline - & 9. ii. 44 & - & 5 & 5 & ++ & ++ & ++ \\
\hline- & 4. viii. 44 & - & 5 & 5 & +++ & $++t$ & ++ \\
\hline- & 9. i. 45 & - & 5 & 5 & +++ & ++ & +++ \\
\hline - & 23. i. 45 & - & 5 & 5 & +++ & + & +++ \\
\hline- & 24. i. 45 & - & 5 & 6 & +++ & ++ & + \\
\hline- & 26. ii. 45 & -- & 5 & 5 & ++ & +++ & ++ \\
\hline - & 26. ii. 45 & - & 5 & 5 & $++t$ & + & ++ \\
\hline- & 12. ix. 45 & - & 5 & 6 & +++ & ++ & ++ \\
\hline \multicolumn{8}{|c|}{ DMP, straight, rubbed } \\
\hline- & 6. iii. 45 & - & 5 & 5 & ++ & + & 0 \\
\hline- & 12. iii. 45 & - & 5 & 5 & ++ & ++ & + \\
\hline- & 12. iii. 45 & - & 5 & 5 & ++ & \pm & 0 \\
\hline- & 14. iii. 45 & - & 5 & 7 & + & $\bar{t}$ & . \\
\hline- & 20. iii. 45 & - & 5 & 5 & +++ & + & + \\
\hline \multicolumn{8}{|c|}{ DMP, straight, protected } \\
\hline 3 & 6. iii. 45 & 75 & 5 & 5 & +++ & + & + \\
\hline 3 & 23. v. 45 & 75 & 5 & 7 & ++ & ++ & ++ \\
\hline 5 & 28. ii. 45 & 65 & 10 & 7 & . & ++ & +++ \\
\hline 7 & 7. iii. 45 & 58 & 5 & 6 & +++ & ++ & + \\
\hline \multicolumn{8}{|c|}{ DMP, clay creams, simple, rubbed } \\
\hline 3 & 7. iii. 45 & 75 & 5 & 6 & + & + & - \\
\hline 3 & 14. iii. 45 & 75 & 5 & 7 & + & + & + \\
\hline 3 & 23. v. 45 & 75 & 5 & 7 & + & + & + \\
\hline \multicolumn{8}{|c|}{ DMP, clay cream, shellac } \\
\hline 11 & 26. iii. 45 & 30 & 5 & 5 & ++ & + & + \\
\hline 24 & 25. iv. 45 & 60 & 5 & 7 & ++ & ++ & +++ \\
\hline 28 & 25. iv. 45 & 45 & 5 & 7 & ++ & ++ & + \\
\hline \multicolumn{8}{|c|}{ DMP, clay creams, white shellac } \\
\hline 38 & 27. vi. 45 & 44 & 15 & 6 & \pm & . & . \\
\hline 38 & 28. vi. 45 & 44 & 15 & 7 & \pm & · & . \\
\hline 39 & 29. v. 45 & .44 & 10 & 5 & ++ & ++ & ++ \\
\hline 42 & 18. vi. 45 & 44 & 15 & 5 & + & . & . \\
\hline 42 & 18. vi. 45 & 44 & 15 & 5 & +++ & . & . \\
\hline \multicolumn{8}{|c|}{ DMP, clay creams, polystyrene } \\
\hline $45^{*}$ & 26. vi. 45 & 78 & 26 & 5 & +++ & \pm & . \\
\hline $50 \dagger$ & 2. vii. 45 & 46 & 15 & 5 & + & + & . \\
\hline \multicolumn{8}{|c|}{ Polystyrene in DMP } \\
\hline $51 \ddagger$ & 2. vii. 45 & 60 & 5 & 5 & +++ & ++ & ++ \\
\hline & $\begin{array}{l}* \text { Spes } \\
\dagger \text { Spes } \\
\ddagger \text { DM }\end{array}$ & $\begin{array}{l}\text { iite } 50 \text { g., } \\
\text { iite } 40 \text { g., } \\
0 \mathrm{ml} . \text { pol }\end{array}$ & $\begin{array}{l}0 \% \text { polyst } \\
0 \% \text { polys } \\
\text { tyrene } 40\end{array}$ & $\begin{array}{l}\text { rene in Dl } \\
\text { rene in DI }\end{array}$ & $\begin{array}{l}0 \mathrm{ml} ., \mathrm{w} \\
0 \mathrm{ml} .\end{array}$ & $5 \mathrm{ml}$ & \\
\hline
\end{tabular}

A paste was then made by addition of $40 \mathrm{ml}$. of $20 \%$ solution of shellac in ethyl alcohol to $58.8 \mathrm{~g}$. paste 5 (paste 11; DMP percentage content v/v 30). This was a liquid which spread readily and left a hard skin on application. It was unsatisfactory as a preparation the clay settling on standing. Paste 24
(Speswhite 30 g., DMP $44 \mathrm{ml} ., 20 \%$ shellac in ethyl alcohol $26 \mathrm{ml}$.; DMP percentage content, 60) gave excellent spread but failed due to excess of DMP to give a firm film on application. Further, though an excellent paste when first made or stirred up, it showed the usual feature of all such clay mixtures of this type 
of setting in a gel-like fashion with some extrusion of fluid. Cream 28 (Speswhite 34 g., DMP $31 \mathrm{ml} ., 20 \%$ solution of shellac in ethyl alcohol $30 \mathrm{ml}$. and arachis oil $5 \mathrm{ml}$., DMP percentage content. 45) fulfilled all requirements as regards spreading qualities and formation of a hard film. It, however, still had the gel character and some syneresis on keeping. All these shellac preparations were also of an unpleasant brown colour. Evaporation of the alcohol was also too rapid to allow of satisfactory leisurely spread.

Pastes were now made using white shellac in butyl alcohol. These were an improvement but were still of the gel type showing some syneresis. Whilst breaking down at once on stirring or application to the skin the 'setting' on standing was objectionable. It was thought that if such preparations could be in some way converted into an emulsion they might be improved and this was found to be brought about by addition of a small quantity of water and stirring.

The change was remarkable, addition of even $1.0 \mathrm{ml}$. of water to $100 \mathrm{ml}$. paste converting the mass from a clay gel to a typical and excellent cream. The three creams 38,39 and 42 were of this character and of the same composition except for the different amounts of tap water added (Speswhite 50 g., DMP $40 \mathrm{ml}$., $20 \%$ white shellac in $n$-butyl alcohol, water $1 \cdot 0,0.5$ and $2.5 \mathrm{ml}$. respectively; DMP percentage content $\mathrm{v} / \mathrm{v}$ 40). These pastes in screw-topped jars remained unchanged for many months and withstood heating at $60^{\circ} \mathrm{C}$. for 7 days.

The skin formed was quite resistant to rubbing and was retained overnight with little change. It could not be removed by washing with soap and water but was readily and quickly removed by a little grease (see below).

Unfortunately results from tests were very indifferent, though pressure of work at the time prevented as much attention being given to such testing as was desirable. Such tests as were made are given in Table 3. The general impression throughout was that though DMP clay creams were effective they seemed somewhat less so than their DMP content should have ensured in all except the simplest form of these creams and that the absorbed fraction is relatively inactive has to be regarded as a possibility.

A number of clay pastes were also prepared containing various proportions of beeswax and white spirit or turpentine as solvent. These were liable to come off in 'rolls' except where very thinly applied and the DMP content was low.

Some experiments with polystyrene were suggestive that some success might be attained by employing this substance in concentrated solution in DMP. Preparation 51 (polystyrene 4 g., DMP $5 \mathrm{ml}$. heated until dissolved on a water bath) was a thick viscous water-clear fluid of about the consistence of glycerine with DMP content of $60 \%$. It spread well, $0.3 \mathrm{ml}$. sufficing to cover 30 sq.in. of skin with

\section{J. Hygiene 45}

rubbing. It left a slightly oily but not sticky or unpleasant film. It gave good results with tests (see Table 3). The polystyrene, however, was thrown out by water on the arm as a milky fluid and it is doubtful how far sweating would make its use feasible.

It was found that a considerably more concentrated solution than the above could be made with prolonged heating and there might be possibilities in the direction of a still more viscous preparation having rub-resistant properties.

\section{(3) Demonstration of rate of disappearance of} DMP from skin by the use of clay films

When a clay-white shellac-DMP preparation such as clay 42 has been applied to the skin as a thin film it forms in a few minutes a dry rub-resistant skin. This is practically invisible.

If now the shellac be dissolved out by alcohol the clay is left as an obvious white deposit on the skin. This white deposit of clay is not removable by soap and water, but is so at once by use of a little oil or grease or by glycerine. It is clear that the clay as left behind on the skin when the shellac has been removed is adsorbed on the surface epithelium.

So long as DMP is present the film remains quite invisible. When, however, DMP (along with the shellac) has been removed by alcohol the clay film remaining shows up white. The same happens in the course of time when it may be assumed the DMP has disappeared by volatilization or absorption. The following observations relate to application of an amount of cream 39 equivalent to 5 cu.mm. DMP to the sq.in. on the back of the forearm.

$6 \mathrm{hr}$. Traces of white only at rubbed corners.

$8 \mathrm{hr}$. Some general whiteness beginning to show.

$12 \mathrm{hr}$. Strongly white at borders and some general whiteness over area.

$21 \mathrm{hr}$. Strongly and opaquely white over whole area.

Since the DMP is restrained from any possible spread and even removal by contact the above would seem to show that 5 cu.mm./sq.in. has in $21 \mathrm{hr}$. disappeared, due either to volatilization or absorption into the skin or both. Disappearance of repellent effect of DMP after application to the skin would appear from recent work in America to be due to absorption by the skin and not to volatilization. That even at much lower temperatures than that of the skin thin fabrics such as butter-cloth when impregnated with DMP to the extent of $5 \mathrm{cu} . \mathrm{mm}$. lose repellent effect in at most $48 \mathrm{hr}$. would seem, however, to be in favour of loss by volatility in the present case. The method, whatever the cause of disappearance, affords a striking means in the case of oily repellents of demonstrating such loss and its extent with different dosage and conditions. 
(4) Result of applying repellent preparations in different ways

In the course of these experiments occasion was had to compare the effectiveness of creams spread by placing the required dose on the arm in a mass and spreading with the palm by the method that has been usually used in tests, viz. distributing the preparation first over the area in drops or blobs and rubbing in as uniformly as possible with the finger. It was evident that in cases where the first method was employed results were often strikingly poorer. The first method is probably effective enough dealing with a thin mobile oily fluid such as DMP applied direct, but with creams distribution may not be so uniform as may be supposed. That vanishing creams, unless used very lavishly, are not uniformly distributed had already been noted. Since in practice the palm method is the only one that could be very well recommended for use with creams of the rub-resistant type it is important to recognize that when using creams in this way, especially where these are not exceptionally spreadable, there is a danger that some parts will not receive adequate dosage. In general, this is a point in favour of application straight as against use of preparations of repellents.

\section{(5) General conclusions regarding rub- resistant creams}

Putting aside small differences such as may be found with different types of ordinary creams, our observations have led us to regard the making of a real rub-resistant cream as beset with grave difficulties.

It has been possible to prepare creams which are physically an advance on any of this type which we have seen and by the use of white shellac greatly to improve their cosmetic appearance. Nevertheless we feel doubtful of their practicability. In the first place any 'shellac' type forming a permanent skin, especially if used constantly, must obviously have drawbacks when used on the face, near hair, etc. More serious is the fact that a good deal of the effectiveness of the DMP content appears to be lost in such preparations.

More hope seemed to lie in the formation of very viscous creams, e.g. by the use of polystyrene in concentrated solution, but here again, though efficiency was not lost to the same extent, breaking down of the film in the presence of water is a serious objection. The problem remains still very far from being solved.

\section{PHYSICAL PROPERTIES IN RELATION TO REPELLENCY}

\section{(1) Boiling-point}

Upon the boiling-point depends very largely the volatility, and upon the volatility the time which a film of repellent will remain on the skin. Upon the volatility will also depend very largely the time for which a fabric impregnated with a given repellent will retain its repellency. The boiling-point is therefore one of the first facts regarding a substance to be tested for repellency which one wishes to know. Such knowledge may save much waste of time in testing substances which could not possibly be useful as repellents.

That very volatile substances like alcohol with boiling-point up to, say, $100^{\circ} \mathrm{C}$. would be useless as repellents is of course quite obvious - a film of water, for example, will have disappeared from the arm in $10 \mathrm{~min}$. It does not seem, however, to have been appreciated how high a boiling-point is necessary before a substance has any chance of lasting, say, $6 \mathrm{hr}$., as a film on the warm skin.

Testing substances as has been done in the Unit at 2, 4 and $6 \mathrm{hr}$. the following may occur.

(1) The substance has disappeared from the skin before the $2 \mathrm{hr}$. test as shown by absence of any visible film, loss of smell previously present and complete absence of any repellent effect. Among such substances, to mention only a few, are: amyl butyrate, b.p. $170-180^{\circ} \mathrm{C}$; methyl cyclohexanyl acetate, b.p. $175-190^{\circ} \mathrm{C}$; methyl benzoate, b.p. 198-200 ${ }^{\circ}$ C.; ethyl benzoate, b.p. 212-215 ${ }^{\circ}$ C. Very many esters and monohydric alcohols of essential oil type fall in this class. It would seem safe to say that in general a substance with a boiling-point below $200^{\circ} \mathrm{C}$. at $760 \mathrm{~mm}$. will not be retained even for $2 \mathrm{hr}$. on the skin and may be disregarded as having any potential use as a repellent in practice. Whether in some cases such substances might have an action if embodied in some base has not been investigated but experience does not suggest such a possibility as likely to have any practical importance.

(2) The substance is still effective in a dosage of, say, 10 cu.mm./sq.in. at $2 \mathrm{hr}$. but fails or falls off greatly at $4 \mathrm{hr}$. Among such substances that have been tested are: diethyl adipate, b.p. $245^{\circ} \mathrm{C}$.; isobutyl phenyl acetate, b.p. $247^{\circ} \mathrm{C}$.; citronellol, b.p. $117-118^{\circ} \mathrm{C} . / 17 \mathrm{~mm}$. (approx. $246^{\circ} \mathrm{C} . / 760 \mathrm{~mm}$.). Here a limit of about $250^{\circ} \mathrm{C}$. would probably in general hold.

(3) The substance is effective to $4 \mathrm{hr}$. but fails at $6 \mathrm{hr}$., the transition often being very abrupt. Among such substances that have been encountered are: di-isopropyl adipate, b.p. $140^{\circ}$ C. $/ 18 \mathrm{~mm}$. (approx. $273^{\circ} \mathrm{C} . / 760 \mathrm{~mm}$.) ; dimethyl 4 tetrahydro-phthalate, b.p. $87^{\circ} \mathrm{C} . / 2 \mathrm{~mm}$. (approx. $273^{\circ} \mathrm{C} . / 760 \mathrm{~mm}$.) and a number of high molecular weight unsaturated monohydric alcohols.

(4) The film is still visible and often very little changed at $6 \mathrm{hr}$. and if effective at all is almost equally effective at 6 as at $4 \mathrm{hr}$. Very near the border here is dimethyl phthalate, b.p. $282-285^{\circ} \mathrm{C}$.

It would seem that somewhere about a boilingpoint of $280^{\circ} \mathrm{C}$. is necessary if a substance is to be 
an effective repellent at $6 \mathrm{hr}$. even when protected from rubbing. Above this are many substances with boiling-points of $300^{\circ} \mathrm{C}$. or more in which no question of lack of permanency of the film arises. With very high boiling-points, over $350^{\circ} \mathrm{C}$., there seems to be a tendency for action to be maintained, but such action is at no time of a very high order.

It is unfortunately just about in the range where the boiling-point is most likely to be useful that a difficulty arises in that owing to decomposition at high temperatures the boiling-point of many substances can only be determined under reduced pressure. As the difference in the values so arrived at and the boiling-point at atmospheric pressure is very considerable it is difficult in such cases off-hand to compare such values and a rough and ready method of converting to values as at $760 \mathrm{~mm}$. pressure is given below.

To make a rough conversion which is sufficiently accurate for the purpose, add 273 to the boilingpoint figure given, multiply the figure so arrived at by the appropriate factor as given below and subtract 273. The result will be very approximately the boiling-point at $760 \mathrm{~mm}$. pressure.

$\begin{array}{cccc}\begin{array}{c}\text { mm. } \\ \text { pressure }\end{array} & \text { Factor } & \begin{array}{c}\text { mm. } \\ \text { pressure }\end{array} & \text { Factor } \\ 1 & 1 \cdot 57 & 10 & 1 \cdot 37 \\ 2 & 1 \cdot 51 & 12 & 1 \cdot 36 \\ 3 & 1 \cdot 48 & 14 & 1 \cdot 35 \\ 4 & 1 \cdot 45 & 16 & 1 \cdot 34 \\ 5 & 1 \cdot 43 & 18 & 1 \cdot 32 \\ 6 & 1 \cdot 42 & 20 & 1 \cdot 31 \\ 7 & 1 \cdot 41 & 40 & 1 \cdot 26 \\ 8 & 1 \cdot 39 & 100 & 1 \cdot 18 \\ 9 & 1 \cdot 38 & 200 & 1 \cdot 12\end{array}$

The factors have been calculated from the formula given in Appendix B, which was a note very kindly given by Dr E. A. Moelwyn-Hughes in commenting on a communication by the writer (Commentary No. 20 to Ministry of Production). The constant $K$ has been taken as 23 , since the value of this increases with the boiling-point and most of the substances for which conversion is required have very high boiling-points. The factor derived is slightly higher if $K$ be taken as 21 as in Dr Moelwyn-Hughes's note.

\section{(2) Melting-point}

In general substances with a relatively high melting-point, i.e. much above $37^{\circ} \mathrm{C}$., have been nonrepellent. The solid, trimethyl citrate, for example, is quite without repellent properties, unlike triethyl, tri-isopropyl and tri-butyl citrate which are oily liquids with strong repellent properties. The rule is not, however, invariable since coumarin m.p. $68-70^{\circ}$ C. has some repellent properties. Some importance attaches to solid repellents since a solid substance is more likely to give prolonged protection when used to impregnate clothing than liquid compounds, should one be found with the necessary repellent properties.

Substances with a melting-point at or near body heat usually give on application to the skin an oily or wax-like film. This may have some advantages as it is probably less liable to loss by creep or rubbing than more mobile fluids.

\section{(3) Viscosity, surface tension and 'creep'}

Viscosity and surface tension are especially of interest in relation to 'creep'. High viscosity also militates against ease of spread, but may have an advantage in giving greater resistance to removal by rubbing from clothing, etc.

The term 'creep' has been used to describe the condition where an oily substance applied to the skin extends at its edges on to untreated areas. If a moderately thick application of a substance like dimethyl phthalate be made over the dorsum of the forearm, within an hour this will have extended at its borders several $\mathrm{cm}$. and after $2 \mathrm{hr}$. more fluid may be present on the under surface of the arm below the wrist than where originally applied.

There appear to be two processes involved in this spread of oily fluids. First 'creep' in which the oil is carried along the sharp re-entrant angles of the skin sulci and later 'flow' where, the surface having been wetted by creep, gravity comes into play with draining of the fluid towards more dependent areas. The importance of these phenomena lies not only in that fluid is removed from the area where it is originally applied, but that by keeping supplied dependent areas where the fluid tends to lie thickly they favour continual removal by contact with clothing, etc.

The nature and extent of creep can be well studied if an oil-soluble dye, such as Sudan III or Sudan black $\mathrm{B}$, is dissolved in the repellent and this then applied over a small marked-out area of skin, say on the back of the forearm. At once, under a low power, fluid will be seen extending along the skin sulci much as if these were small irrigation channels. Later the raised areas of skin between the sulci become wetted. The extent of area occupied in a given time varies with different repellents (see Fig. 4). The following technique has been used for measuring and keeping permanent records of the phenomenon.

A rectangular area $2 \times 1$ in. on the back of the forearm is marked by four spots of ink at the corners of a template. A given quantity of repellent coloured with the dye (usually 10 cu.mm./sq.in.) is then spread over the area using a fine rounded glass rod and the time noted. After the desired interval of time (15 min. or longer) a sheet of white paper of convenient size in which a hole has been cut corresponding to the area is then carefully laid on the arm so that the corners of the hole correspond with 
the ink spots, the whole covered with a sheet of soft cardboard and pressed upon the arm. A perfect impression may thus be obtained showing the area over which creep has extended. A line drawn round the edge of the stained area gives a permanent record which can be traced off on squared paper and the area occupied measured. The number of times the area of spread is greater than the originally determined by an Ostwald's viscometer and surface tension by weight of a known number of drops from a bulb stalagmometer as compared with water.

It will be seen that most of the repellents show surface tension lying between 0.5 and 0.4 of water as 1.0 and that variations in the creep index have little or no relation to such differences as there may be in surface tension. No doubt a low surface tension

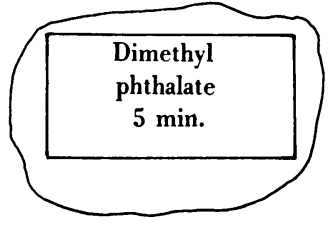

A

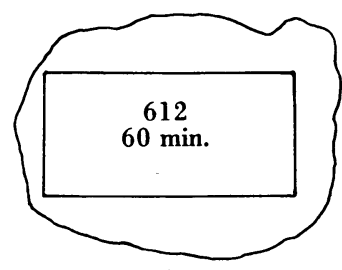

D

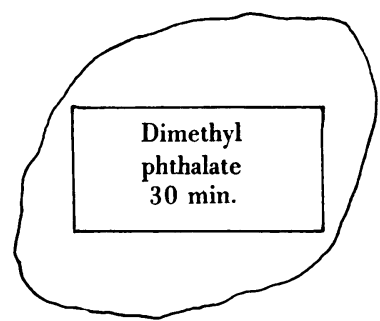

B

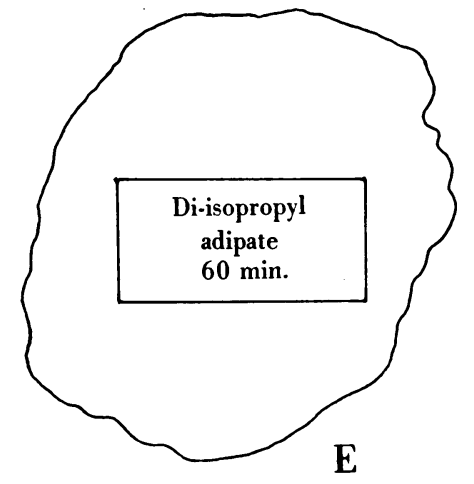

$\mathrm{E}$

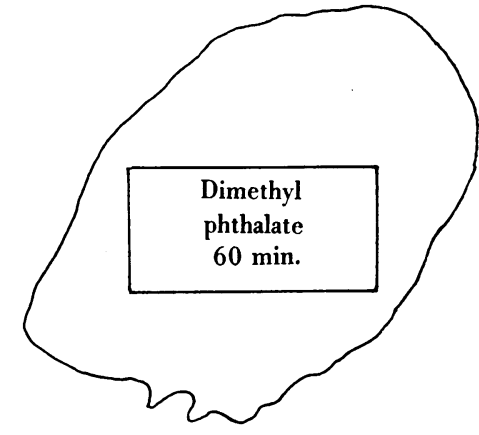

C

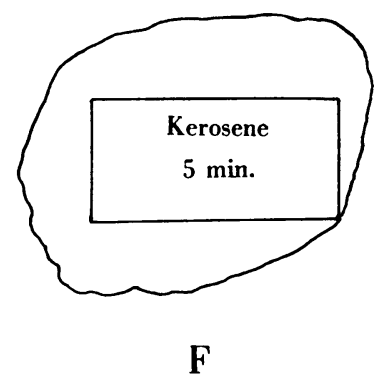

F

Fig. 4. Showing tracings of creep as made by the method described in the text. The outline shows extent of arm surface covered by the time noted. The rectangle is the area originally treated with repellent (in this case rectangles measure $2 \times 1$ in. and the dose was $20 \mathrm{cu} . \mathrm{mm}$. or $10 \mathrm{cu} . \mathrm{mm}$./sq.in. as usually employed when testing). A. Dimethyl phthalate at $5 \mathrm{~min}$. B. The same substance at $30 \mathrm{~min}$. C. The same at $60 \mathrm{~min}$. D. Rutger's 612 at $60 \mathrm{~min}$. E. Di-isopropyl adipate at $60 \mathrm{~min}$. F. Kerosene at $5 \mathrm{~min}$.

treated area in some fixed time interval gives a convenient index figure.

Washing the arm with soap and water greatly reduces the extent of spread and in order to enable experiments to be carried out consecutively and to provide as uniform conditions as possible the following was the procedure adopted. The arm was washed with soap and water. A single drop of arachis oil was then placed in the middle of the upper surface of the forearm and thoroughly rubbed over the arm with the palm of the hand for half a minute by the stop watch. This gave results very similar to unwashed skin and relatively repeatable.

In Table 4 are given a number of determinations of viscosity and surface tension of repellents with creep index as determined above. Viscosity was is essential for creep to take place but otherwise it seems to play but a small part in determining the rate of spread.

Viscosities vary enormously and it is clear that it is the viscosity which mainly determines the value of the creep index. Indeed for practical purposes the relationship is so close that the viscosity would be a sufficient guide and one which could be readily determined. Plotting creep index as determined at 30 min. against log viscosity in relation to water as 1 , the following relationship holds for the present series:

$$
\text { Creep index }=4 \cdot 8-1 \cdot 6 \log \text { viscosity. }
$$

With Rutger's 612 on this basis, however, the value given is rather low. 
Table 4

\begin{tabular}{|c|c|c|c|c|c|c|c|c|c|c|}
\hline \multirow[b]{3}{*}{ Substance } & \multirow[b]{3}{*}{$T^{\circ} \mathrm{C}$. } & \multirow{3}{*}{$\begin{array}{c}\text { Density } \\
\text { to } \\
\text { water } \\
\text { as 1 }\end{array}$} & \multirow{3}{*}{$\begin{array}{c}\text { S.T. } \\
\text { to } \\
\text { water } \\
\text { as } 1\end{array}$} & \multicolumn{2}{|c|}{ Viscosity } & & & & & \\
\hline & & & & \multirow{2}{*}{$\begin{array}{c}\text { Time } \\
\text { in } \\
\text { sec. }\end{array}$} & \multirow{2}{*}{$\begin{array}{c}\text { To } \\
\text { water } \\
\text { as l }\end{array}$} & \multicolumn{5}{|c|}{ Creep index at } \\
\hline & & & & & & $5^{\prime}$ & $10^{\prime}$ & $15^{\prime}$ & $30^{\prime}$ & $60^{\prime}$ \\
\hline DMP & $18 \cdot \tilde{5}$ & $1 \cdot 196$ & 0.57 & 510 & 13 & $1 \cdot 80$ & $2 \cdot 28$ & $2 \cdot 74$ & $3 \cdot 11$ & 3.90 \\
\hline Rutger's 612 & $18 \cdot 5$ & 0.946 & $0 \cdot 43$ & 11100 & 292 & - & - & - & 1.72 & $2 \cdot 09$ \\
\hline Indalone & $18 \cdot 5$ & $1 \cdot 055$ & 0.44 & 1558 & 41 & - & 1.79 & $1 \cdot 83$ & $2 \cdot 20$ & $2 \cdot 50$ \\
\hline$(134)^{*}$ & 18.5 & 1.000 & - & 1260 & 33 & - & - & - & - & $2 \cdot 85$ \\
\hline$(135)^{*}$ & $18 \cdot 5$ & 0.970 & $0 \cdot 40$ & 4463 & 117 & - & - & - & 1.70 & - \\
\hline$(136)^{*}$ & 18.5 & - & - & 2920 & 77 & - & - & - & 1.62 & - \\
\hline$(137)^{*}$ & 18.5 & - & - & 2462 & 65 & - & - & - & 1.73 & - \\
\hline$(139)^{*}$ & 18.5 & - & - & 2057 & 54 & - & - & - & $2 \cdot 02$ & - \\
\hline$(140)^{*}$ & $20 \cdot 0$ & - & 0.50 & 367 & 10 & - & - & - & 3.00 & - \\
\hline Diethyl phthalate & $20 \cdot 0$ & $1 \cdot 123$ & $0 \cdot 49$ & 390 & 11 & - & - & - & - & - \\
\hline Tri-isobutyl citrate & $20 \cdot 0$ & - & _- & 4140 & 112 & - & - & - & - & $2 \cdot 67$ \\
\hline Di-isopropyl adipate & $20 \cdot 0$ & $0 \cdot 947$ & $0 \cdot 44$ & 145 & 4 & - & - & - & 3.85 & - \\
\hline Kerosene & 18.5 & 0.828 & $0 \cdot 30$ & 70 & 2 & $2 \cdot 46 \dagger$ & - & - & - & - \\
\hline
\end{tabular}

* These substances are a series of higher alcohol phthalates that have been reported upon by the Unit.

$\dagger$ So far no repellent has shown such rapid extension of creep ( $2 \frac{1}{2}$ times the original area of 2 sq.in. in 5 min.).

\section{(4) Specific gravity}

Some determinations for specific gravity at temperature $18-20^{\circ} \mathrm{C}$. are given in the column under density in Table 4. The only remark necessary under this head is in respect to DMP which has a specific gravity of approximately 1.2 and so sinks rapidly in water, most oily repellents being lighter than water.

\section{ON AN APPARATUS FOR MEASURING REPELLENCY AT A DISTANCE AND SOME OTHER REACTIONS OF MOSQUITOES}

\section{(1) The apparatus}

The following simple compact and workable apparatus has been found useful for studying certain reactions of Aedes aegypti and for quantitative determinations of the extent to which different repellent substances act on contact or at a distance.

The apparatus consists of a glass tube $52 \mathrm{in.} \mathrm{long}$ and $3 \frac{1}{2}$ in. in diameter so mounted on a light wooden stand with supports that it can be rotated on its axis. It is held in place by soft straps which buckle over the tube. These straps are glued on to the supports with a felt lining thus making rotation smooth and easy (Fig. 5, C).

Into the tube, when in use, is slipped a strip of cartridge paper of such a length that it occupies the whole length of the tube from the open end at $b$ to the cardboard collar at $a$, and of such a breadth that when placed in the tube it leaves a window for observation of convenient width. The strip in the apparatus used by the writer measures $24 \frac{3}{4} \times 8 \frac{1}{4}$ in. $(63 \times 21 \mathrm{~cm}$.) leaving a window along the whole length of the tube about $5 \mathrm{~cm}$. wide. The paper strip is divided by lines across its length into six equal parts, labelled prominently with large figures placed near the median line and starting from right to left. It is supported when in position by three celluloid circles made from strips of celluloid sheet by cementing these into rings with ethyl acetate and used as described later. A further celluloid ring of this kind will be required, this time to fit over the tube to hold a piece of mosquito netting in place closing the end $b$.

At the end $a$ is a cardboard collar of a depth equal to that of the cylindrical container to be described, preferably about 3 in. deep with a flange $\frac{1}{2}$ in. wide at its outer end to limit the extent to which it can be pushed into the tube. This is readily made by pasting or folding over a $\frac{1}{2}$ in. wide strip of cardboard along one edge of a piece of cardboard of a width equal to the depth of the cylindrical container and of a length so that its ends just meet when it is pressed against the inner surface of the tube. The cardboard collar should be of such a thickness that the cylindrical container when pushed into it when in position in the tube is held firmly but easily. When adjustments are correct the collar can be finally held together by a strip of adhesive plaster round the flange.

The cylindrical container is prepared froma syrup or other tin with a press-down lid. Towards one side of the lid is soldered in a length of brass tube hermetically closed at the inner end and of such a size as to take one of the small cylindrical electric lamps obtainable on the market, its outer end being fitted with a screw ring to take the lamp attachment. The lamp flex is provided with a push on-and-off switch. There should be a slit or small hole towards the outer end of the tube so that it can be seen when the lamp is lit. The brass tube is soldered in towards the side of the press-down lid to leave space for two soldered 


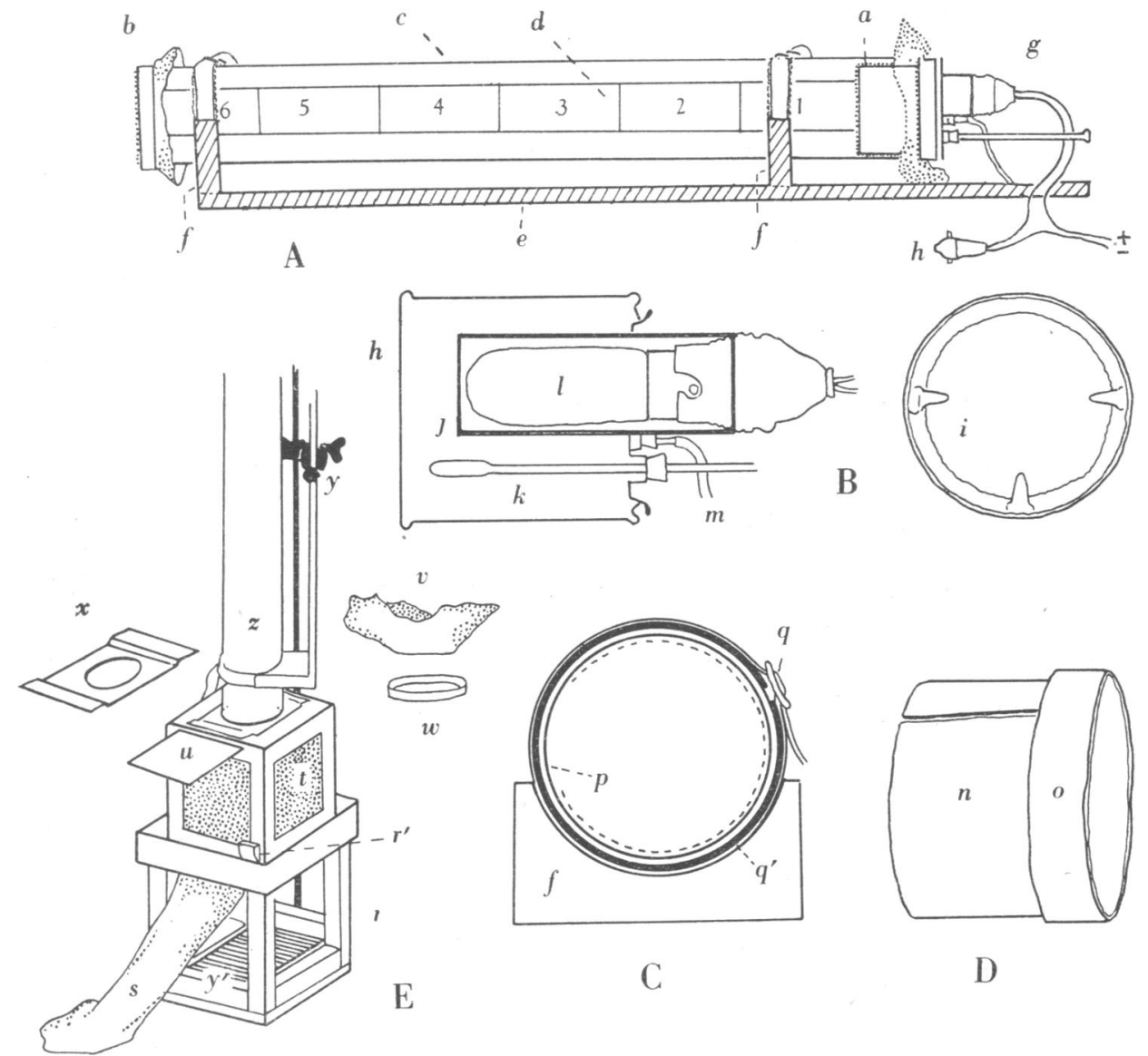

Fig. 5. A. General view of apparatus. $a$, warm chamber and collar in position with netting pushed in as shown by dotted line. Removal of the warm chamber leaves the collar with netting in position; $b$, netting with celluloid ring over left end of tube; $c$, glass tube containing paper divided into numbered sections as seen through $d$; $d$, window left by gap due to paper sheet not completely covering mouth of tube with figures on far side plainly visible; $e$, light board with supports $f, g$, lamp circuit, etc.; $h$, on-and-off switch in circuit. B. Showing structure of warm chamber. $h$, syrup tin; $i$, bottom of syrup tin with tags soldered on to hold filter paper in position; $j$, piece of brass tubing sealed off and provided with screw to take lamp socket. It is soldered into the press-on lid of the syrup tin; $k$, thermometer; $l$, lamp; $m$, overflow tube. C. Showing method by which glass tube is fixed on stand. $f$, wooden support ( $f$ of general view); $p$, glass tube fixed with soft strap $q^{\prime}$ and buckle $q$. The strap with felt on inner side is glued on to the wooden support. The dotted line inside the tube indicates the paper sheet leaving gap for observation. D. Cardboard collar. $n$, portion fitting closely into end of glass tube but just tight enough to allow a piece of mosquito net to be carried in, thus closing the end of the tube with a netting diaphragm; $o$, sleeve limiting extent to which collar can be pushed into tube. E. Loading device. $r$, wooden support taking metal foot of large retort stand and with raised wooden edge and wedge $r^{\prime}$ to take an emergence cage; $s$, sleeve of emergence cage; $t$, emergence cage; $u$, sliding zinc door of emergence cage pulled out after tube and zinc diaphragm $x$ is in position; $v$, mosquito netting; and $w$, celluloid ring slipped over $v$, when tube has been loaded; $y$, clamp of retort stand holding base board of apparatus in position; $y^{\prime}$, foot of retort stand; $z$, tube of apparatus in position for loading.

in tin collars to take respectively a short thermometer (those used for incubators are very suitable) and a piece of glass tubing to act as escape for excess water when the container is heated. A length of thin rubber tube is attached to the glass tube to carry away such surplus. Finally there are soldered to the bottom of the tin three small metal tongues to hold in place a small filter paper which can be wetted or not and on which a measured quantity of repellent can be placed. When completed the container is filled with water.

The apparatus is assembled by attaching a celluloid ring to one end (that with the figure 1) of a cartridge paper strip using a wire paper fastener. 
The end of the paper is then rolled round the ring and pushed into the tube from the end $b$. When halfway in a second celluloid ring is inserted into the rolled paper and this pushed fully home until it meets the cardboard collar. The third celluloid ring is then inserted. Finally a piece of mosquito netting is placed over the end $b$ of the tube and fixed in place by the fourth ring. The whole operation takes at most a minute or two. It is of course desirable to use a fresh cartridge paper whenever there is any chance that the one that has been used has become contaminated with a repellent or its smell. Once the correct size of the paper has been ascertained and one strip made any number of others can be readily prepared with this as a model.

Having closed end $b$ of the tube, a second piece of mosquito netting is laid over end $a$, and the cardboard collar pushed in, thus sealing the tube with mosquito netting at either end.

Meanwhile the lamp in the container is switched on and the water heated up to the desired temperature, taking care to rotate the container from time to time to ensure uniform heating. It is easy to maintain the desired temperature by occasional use of the switch.

\section{(2) Method of use}

To load the apparatus s.c. cages as described in the section on rearing Aedes aegypti are used with the same precautions regarding standardization of the mosquitoes. Any desired sex or number can be arranged for at pupa-collecting time and any desired age of mosquito tried out. By standardizing results in this way many pitfalls are avoided.

To transfer mosquitoes from these cages to the apparatus a loading device was found very helpful. This consisted of a wooden frame with a large retort stand arranged as in Fig. 5, E. The top of the frame was of a size to fit the sleeve end of an s.c. cage and was provided with a flange and some wedges so that the cage could be fixed firmly in place with the sleeve hanging down into the frame. Over the sliding door of the cage (now facing upwards) is placed a shutter, designed to allow the cage door to be opened without mosquitoes escaping and with an opening to fit the end of the apparatus tube when this is lifted bodily and clamped in position over the aperture as shown in the figure. On opening the shutter and manipulating the sleeve as many mosquitoes as desired can be caused to fly up into the tube. About fifty is a suitable number as it is difficult to count larger numbers sufficiently rapidly when conducting the experiment.

Before an experiment the apparatus duly set up with its complement of female mosquitoes is placed in a suitable situation, preferably under equalized illumination in a constant temperature room, the tube rotated so that the window is suitably placed for observation and so that no direct light shines into the tube, and left for the mosquitoes to settle down. Usually half an hour has been allowed for this, but a smaller time would probably suffice. Meanwhile the lamp in the container (which has not yet been inserted) has been turned on to heat the water to the desired temperature.

Before an experiment has been started a preliminary count has usually been made of the mosquitoes on the netting at $a$, in the six different numbered compartments and on the netting at $b$. There has always been a tendency for the numbers on the nettings at the open ends of the tube to be larger than in the middle compartments, but on the whole the distribution is fairly uniform. Table 5 shows counts that have been made in this way. It is not always possible to be quite accurate, but with a little practice and especially with two observers rapid counts can be made without difficulty at any given time limit which are very nearly so.

Seeing that the index referred to later is based on the sum of 16 of such figures, i.e. readings of $a, 1,2$ and 3 at $2,5,10$ and $15 \mathrm{~min}$. divided by the sum of another set of 16 , viz. $4,5,6$ and $b$, irregularity of distribution may be largely disregarded. Taking this index on normal counts (Table 5) it works out at 1.04 or as expressed to one place of decimals $\pm 1 \cdot 0$.

When performing an experiment the container is inserted at the desired temperature, usually $40^{\circ} \mathrm{C}$., and counts as previously noted made at $2,5,10$ and $15 \mathrm{~min}$. If a repellent is being tested a small quantity, usually $0.4 \mathrm{ml}$., is dropped on the filter paper disk and allowed to permeate this before the container is introduced.

In order to get a figure to indicate degree of repellency (or attraction) the counts at $2,5,10$ and 15 min. at $a, 1,2$ and 3 and at $4,5,6$ and $b$ were added and whichever was the larger sum divided by the other, the index so obtained being given a plus or minus sign according to which form the operation took. In the present case repellency has been indicated by plus and attraction by minus. The two experiments in Table 6 giving respectively the normal reaction to the heated container and repellency by $0.4 \mathrm{ml}$. citronellal illustrate the method.

Usually, as will be seen, repellents like dimethyl phthalate do not give such a pronounced result as citronellal, the index being as a rule $+2 \cdot 5$ or under. The response, however, is more striking than such figures indicate, the effect being added to by the crowding of actively prodding mosquitoes on $a$ in the absence of repellency and the complete change in distribution in the presence of the repellent. Very characteristic too are rapid flights towards $a$ with reversal whilst still on the wing on reaching say compartment 2 . With an index of $+1 \cdot 0$ there must be some degree of repellency since the attraction of the warm container has been overcome but it was not 
Table 5

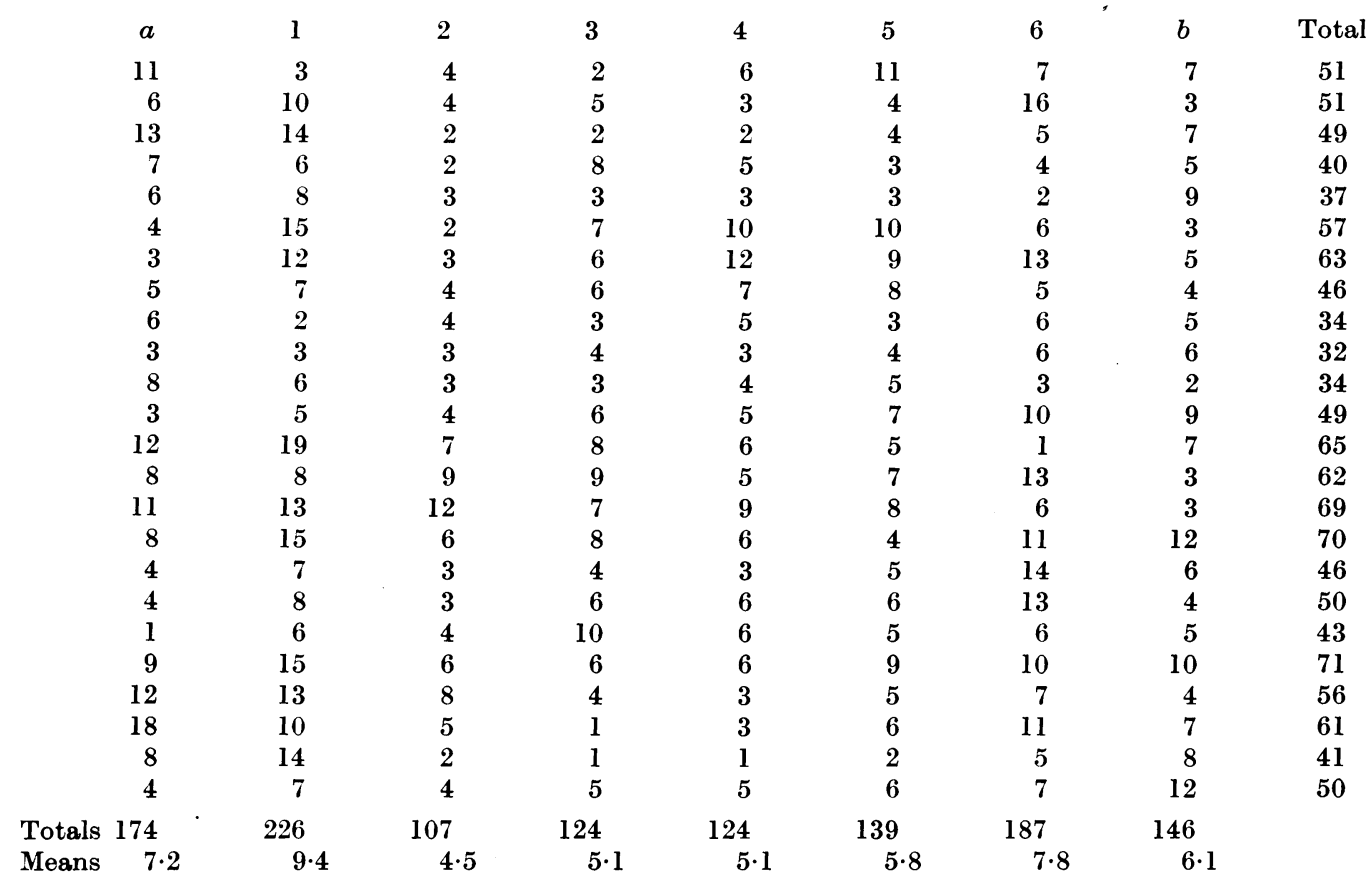

Table 6

Normal reaction Minutes

$\begin{array}{rrrr}2 & 5 & 10 & 15 \\ 19 & 21 & 30 & 26 \\ 5 & 2 & 5 & 12 \\ 3 & 4 & 2 & 3 \\ 2 & 6 & 4 & 4 \\ 5 & 4 & 2 & 1 \\ 6 & 3 & 1 & 1 \\ 6 & 6 & 3 & 2 \\ 6 & 6 & 6 & 6 \\ & \text { Index }-2 \cdot 3 & \end{array}$

$0.4 \mathrm{ml}$. citronellal Minutes

\begin{tabular}{|c|c|c|c|}
\hline 2 & 5 & 10 & 15 \\
\hline 0 & 0 & 0 & 0 \\
\hline 0 & 0 & 0 & 1 \\
\hline 1 & 0 & 0 & 1 \\
\hline 0 & 0 & 0 & 1 \\
\hline 0 & 0 & 0 & 0 \\
\hline 8 & 7 & 0 & 0 \\
\hline 16 & 19 & 19 & 19 \\
\hline 21 & 18 & 28 & 26 \\
\hline
\end{tabular}

wished to make too complicated an index or suggest greater numerical accuracy than such a figure probably possesses. As will be seen, DDT did not exhibit even this degree of repellency and in two trials gave a value equal to the normal reaction.

\section{(3) Effect of heat, moisture and sex}

In Table 7 are given the results of certain experiments in the absence of any repellent.

It is clear in the first place that the attraction exercised by the warm container is not exhibited with males. On the contrary the figures so far as obtained even suggest some slight repellent effect.

The response of females was fairly constant with an index from $2 \cdot 0$ to $2 \cdot 5$.

The effect of moisture (wetting the filter paper disk) under the conditions was not very marked, though the figures were generally a little higher. Moisture alone with mosquitoes at $25^{\circ} \mathrm{C}$. and humidity $90 \%$ in the absence of warmth has no attractive effect. 


\section{(4) Effect of different repellents}

In Table 8 are given results obtained with some repellents. From observations made when carrying out tests it was suspected that repellents varied a good deal in the extent to which they acted at a distance or on contact only. This is shown to be the case though all repellents tried have shown some repellent effect at a distance. This applies to indalone, which though with a smaller index than DMP or Rutger's 612 still shows some repellency. The
It would seem that even with the very strong preparations used repellency is very slight. There seemed to be a very slight toxic effect at a distance with pyrethrin I, but no evidence of this was obtained with the second concentrate containing $65.8 \%$ of pyrethrin II.

\section{(5) General considerations}

It is claimed that the apparatus described is handy, compact and workable, easily allowing for assembly and de-assembly for the purpose of cleaning

Table 7

\begin{tabular}{lccccc} 
Sex & Age & $\begin{array}{c}\text { Sum of } \\
a, 1,2,3\end{array}$ & $\begin{array}{c}\text { Sum of } \\
4,5,6, b\end{array}$ & Index & Remarks \\
Female & 5 & 137 & 56 & $-2 \cdot 5$ & Tube horizontal, dry \\
Female & 5 & 244 & 88 & $-2 \cdot 8$ & Tube horizontal, wet \\
Female & 5 & 185 & 92 & $-2 \cdot 0$ & Tube vertical, dry \\
Female & 5 & 159 & 66 & $-2 \cdot 4$ & Tube vertical, wet \\
Female & 5 & 78 & 36 & $-2 \cdot 2$ & Tube vertical, heat above, wet \\
Female & - & 155 & 65 & $-2 \cdot 4$ & Tube vertical, heat below, dry \\
Male & 5 & 101 & 110 & $+1 \cdot 0$ & Tube horizontal, dry \\
Male & 5 & 84 & 81 & $+1 \cdot 0$ & Tube horizontal, dry \\
Male & 5 & 102 & 145 & $+1 \cdot 42$ & Tube horizontal, dry \\
Male & 5 & 87 & 177 & $+2 \cdot 03$ & Tube horizontal, wet \\
\multicolumn{5}{c}{ Mean index females $-2 \cdot 4}$. \\
\multicolumn{5}{c}{ Mean index males + $\cdot 4.4$}
\end{tabular}

Table 8

\begin{tabular}{|c|c|c|c|c|c|c|}
\hline Substance & Date & Cage no. & Age* & $\begin{array}{c}\text { Sum of } \\
a, 1,2,3\end{array}$ & $\begin{array}{c}\text { Sum of } \\
4,5,6, b\end{array}$ & Index \\
\hline Dimethyl phthalate & 29. xi. 44 & 651 & 7 & 137 & 212 & +1.5 \\
\hline Rutger's 612 & 16. i. 45 & 684 & 6 & 75 & 188 & $+2 \cdot 5$ \\
\hline Indalone & 11. i. 45 & 679 & 7 & 81 & 115 & +1.4 \\
\hline DDT & 17. i. 45 & - & 7 & 179 & 84 & $-2 \cdot 1$ \\
\hline DDT & 17. i. 45 & - & 7 & 171 & 77 & $-2 \cdot 2$ \\
\hline Diethylxanthogen & 23. i. 45 & 690 & 5 & 19 & 244 & $+13 \cdot 0$ \\
\hline A diol (182) & 23. viii. 45 & 889 & 7 & 56 & 72 & $+1 \cdot 3$ \\
\hline Citronellal & 15. i. 45 & 682 & 5 & 4 & 181 & $+45 \cdot 2$ \\
\hline 'Muckensalbe' & 19. ix. 45 & 933 & 7 & 58 & 163 & $+2 \cdot 8$ \\
\hline
\end{tabular}

instance of citronellal strikingly demonstrates the difference between repellency as shown by the essential oils and that by the non-volatile type now chiefly used.

Particular interest attaches to the observations made upon the pyrethrins by this method. In Table 9 are given results with 'colourless extract $40 \%$, Stafford Allen and Co.' and by two concentrates of pyrethrins for which I am indebted to Dr T. F. West of the above firm. These two concentrates given as pyrethrin I and pyrethrin II in the table contain respectively pyrethrin I $59 \cdot 2 \%$, pyrethrin II $13.0 \%$ and pyrethrin II $65.8 \%$, pyrethrin I $18.9 \%$. th 3 tube and replacing all parts, such as the paper, which might be liable to contamination. With the loading device and use of cages and technique as described under testing it enables standardized mosquito cultures of any age, sex or condition to be examined. It gives results which are to a certain extent expressed by a figure.

The chief criticism that has been made is that there is no fixed vapour gradient. But whilst this might be desirable for certain purposes it is not certain that this is so for the object for which the apparatus was designed. It seems almost certain that Aedes aegypti is guided in the first place to its prey (apart from possible light contrast, movement, 
etc., which may play some part) by warm convection currents rather than a heat gradient in the usual sense. It is most probably such a current started by the warm container which passing along the upper portion of the tube causes mosquitoes that sense it to fly towards the container. We have then an attraction set up and a holding force at the end when the near presence of the container excites prodding (effort to puncture). Against this is pitted the repellent power of the substance that is being out of place, but the following results of testing fabrics by this Unit may be recorded.

\section{(1). Method of testing}

The technique used in testing was very similar to that employed for testing repellency of compounds and their preparations when applied to the skin. Suitably shaped samples of the fabric were either laced over the back of the forearm, or simply laid on and wound round spirally with a length of string

Table 9

\begin{tabular}{|c|c|c|c|c|c|c|c|}
\hline Substance & Date & Cage & Age* & $\begin{array}{c}\text { Sum of } \\
a, 1,2,3\end{array}$ & $\begin{array}{r}\text { Sum of } \\
4,5,6, b\end{array}$ & Index & Remarks \\
\hline \multicolumn{8}{|c|}{ Pyrethrum $40 \%$ extract: } \\
\hline Without contact & 29. xi. 44 & 653 & 7 & 100 & 192 & $+1 \cdot 9$ & Note 1 \\
\hline With contact & 29. xi. 44 & 653 & 7 & 54 & 170 & $+3 \cdot 1$ & Note 2 \\
\hline \multicolumn{8}{|l|}{ Pyrethrin I: } \\
\hline \multirow[t]{3}{*}{ Without contact } & 19 ix. 45 & 937 & 7 & 117 & 104 & $+1 \cdot 1$ & Note 3 \\
\hline & 2. x. 45 & 947 & 6 & 80 & 67 & $+1 \cdot 2$ & Note 4 \\
\hline & 3. x. 45 & 948 & 7 & 69 & 41 & $+1 \cdot 7$ & Note 5 \\
\hline With contact & 3. x. 45 & 948 & 7 & 66 & 53 & $+1 \cdot 2$ & Note 6 \\
\hline \multicolumn{8}{|l|}{ Pyrethrin II: } \\
\hline Without contact & 9. x. 45 & 949 & 6 & 174 & 107 & $-1 \cdot 6$ & Note 7 \\
\hline With contact & 9. x. 45 & 950 & 6 & 51 & 58 & $+1 \cdot 1$ & Note 8 \\
\hline
\end{tabular}

Note $1.0 \cdot 1 \mathrm{~g}$. in chloroform on filter paper hung up $3 \mathrm{hr}$. before use. Slight repellency but no sign of lethality or toxic effect.

Note 2. Affected mosquitoes counted where located. The same paper as in note 1, but paper placed at 2 mm. from netting (contact by tip of proboscis). One mosquito on back at 5 min., three at 10 min., and fourteen at $15 \mathrm{~min}$. dead or severely affected.

Note 3. $0.1 \mathrm{~g}$. in chloroform on paper hung up for $3 \mathrm{hr}$. before use. Although somewhat attracted, mosquitoes did not stay for any length of time on netting, i.e. slight repellency when near paper. No lethal or toxic effect.

Note 4. Paper as in note 3 , but kept in dark for 13 days. Had slight smell. Towards end of experiment a few mosquitoes seemed slightly affected.

Note 5. Fresh paper as in note 3. Smelt strongly of pyrethrum. At end of experiment majority of mosquitoes seemed slightly affected and two were on back. $2 \mathrm{hr}$. later mosquitoes removed from apparatus appeared normal or at most somewhat less active.

Note 6. Affected mosquitoes counted where located. Same paper as in note 5, but exposed to settling. Mosquitoes did not hesitate to settle, but at $1 \frac{1}{2} \mathrm{~min}$. one seen on back, at $2 \mathrm{~min}$. a number affected, by end of experiment all but one mosquito out of thirty-four in the chamber knocked out. Floor of chamber littered later with fragmented limbs (autotomy).

Note 7. $0 \cdot 1 \mathrm{~g}$. in chloroform on paper and hung up for $3 \mathrm{hr}$. before use. At most some diminished attraction even if this certain. No lethal or toxic effect after $30 \mathrm{~min}$. in apparatus.

Note 8. Same paper as in note 7 but exposed to settling. Several mosquitoes falling about at 3 min., one on back at $5 \mathrm{~min}$., seven down at $10 \mathrm{~min}$., fifty at $15 \mathrm{~min}$. Chamber floor later littered with fragmented limbs.

tested. It is desired to know how effective this is at a distance when smeared on warm skin rather than the effect of a vapour gradient. Even if it is deficient in this respect for other purposes, its simplicity and workableness may assist towards other apparatus designed for the study of other mosquito reactions.

\section{IMPREGNATION OF FABRICS}

Much work has been done in America and elsewhere in relation to the impregnation of clothing with repellents both in the laboratory and in the field. Any attempt to summarize this work would here be making the spirals about $3 \mathrm{~cm}$. apart. The arm so prepared was then enclosed in the metal shield as used in the repellency tests, bandaged to exclude all biting except through the fabric and inserted in the usual way into a test cage with the usual 100 female and 100 male standardized Aedes aegypti. The area exposed as before was 30 sq.in. and exposure for 15 min. Where the sample of fabric was too small to use in this way a metal cover with an aperture of known area was slipped over the arm and fabric and bandaged as before. A record was kept at the end of the usual intervals of the number settling and of wheals produced. 
For impregnation, except where otherwise stated, pieces of the fabric of a shape and size suitable for covering the arm and cut on a template (area 65 sq.in.) were loosely folded and rolled into a cylinder which fitted loosely into a corked tube. Solvent was then added until after standing a short time no excess seeped out. On the basis of the amount of solvent retained a suitable concentration of the repellent in the solvent (usually carbon tetrachloride) was made and the dried fabric again saturated in the tube, allowed to wait for a time, picked out, unrolled and kept moving without loss of repellent until dry when it was hung up on a line until required for testing.

In the case of netting the same procedure was adopted except that where it was tested for penetration at a distance from the arm a metal box 4 in. square in cross-section and long enough to take the hand and arm was used, the roof and one side (that opposite the observer) being left open with a clipping dimethyl phthalate and Rutger's 612 were surprisingly similar. A dosage of $20 \mathrm{mg}$./sq.in. was about the maximum that could be used with the fabric in question. $15 \mathrm{mg}$./sq.in. of these two repellents, giving protection for about a fortnight, corresponds roughly to the $2.0 \mathrm{ml}$./sq.ft. employed in practice by the American authorities.

Results with other substances than the two mentioned were insufficient to base conclusions upon, but so far as they go they indicate a rather longer protection period for indalone and the solid diethylxanthogen, though in both these cases mosquitoes showed much more tendency to alight for short periods without necessarily biting. In some later tests with fabric impregnated with $1.0 \%$ DDT there was no protection.

\section{(3) Effect of nature of fabric}

Using a fixed concentration in solvent or emulsion different fabrics dipped and hung up to dry do not all

Table 10

\begin{tabular}{lcl}
\multicolumn{1}{c}{ Repellent } & $\begin{array}{c}\text { No. of } \\
\text { tests }\end{array}$ & $\begin{array}{l}\text { Degree of } \\
\text { protection } \\
\text { Dimethyl phthalate }\end{array}$ \\
Rutger's 612 & 38 & $\begin{array}{l}\text { Complete } \\
\text { Considerable } \\
\text { Complete }\end{array}$ \\
Indalone & 17 & $\begin{array}{l}\text { Considerable } \\
\text { Complete }\end{array}$ \\
Diethylxanthogen (solid) & 7 & Complete \\
K. 30 (solid) & 8 & Complete \\
Thanite & 3 & - \\
Benzyl benzoate & - & -
\end{tabular}

arrangement by which the netting could be held in place. This gave a horizontal stretch of the netting (roof) and a vertical stretch (side), there being some difference in the behaviour of the insect under various conditions of repellency which were of some interest in this respect.

\section{(2) Protection period given by different repellents}

A series of some 130 tests were made with cheese cloth impregnated by Dr J. R. Busvine at the London School of Hygiene with known doses of different repellents some with the aid of a volatile solvent and some sprayed in a spraying tower. These were hung up for known periods at summer room temperature of $16-24^{\circ} \mathrm{C}$. and tested by the technique described above. The results are given in the table.

The cheese cloth when untreated or when used only with the solvent offered no protection at all to biting. Results with impregnation by solvent and by spraying were slightly in favour of the former. Sleeves of muslin gave slightly longer protection than small pieces a few inches square. Results with
Number of days protected with dosage mg./sq.in. as under

$\begin{array}{rrrrrr}20 & 15 & 10 & 5 & 2 & 1 \\ 14 & 12 & 10 & 8 & 6 & 4 \\ 21 & 18 & 14 & 10 & 7 & 5 \\ 14 & 12 & 10 & 8 & 6 & 4 \\ 21 & 18 & 14 & 12 & 8 & 6 \\ - & 20 & - & - & - & - \\ 20 & 16 & 13 & - & - & - \\ 29 & - & 20 & - & - & - \\ - & - & - & 6 & - & -\end{array}$

necessarily receive the same dosage of repellent, nor is the maximum amount of repellent which can be used without giving rise to an obvious 'oiled' effect the same. This is illustrated in Table 11 where fabrics were treated with fixed concentration of solvent or with emulsion of dimethyl phthalate with soap as practised in the field.

Percentage of DMP in the solvent was $13 \%$ and in the emulsion $5 \%$. The days of protection are approximate as the fabrics were not tested daily.

Use of the soap emulsion was found to be simple to carry out but even with the best soaps the amount of DMP in permanent emulsion was very small, most being in the form of small globules which settled rather rapidly. Results were much more variable as regards protection period than when solvent wasused.

When carrying out such tests it was observed that so long as pieces of fabrics smelt of DMP, however faintly, they were repellent. In this way a very good selection could have been made without testing against mosquitoes of those pieces which had lost repellency through keeping. 
Briefly the maximum dose that can be employed without making the fabric 'oiled', the dose that the fabric will take up with any given concentration in solvent or emulsion and presumably the protection period will to an appreciable extent (up to 2-3 times) depend on the nature of the fabric. In regard to the protection period insufficient information was obtained to say how far protection with different fabrics is necessarily related to the dosage calculated on area or on weight of the fabric.
Even with saturated netting 47 there was not complete protection against penetration though very nearly so up to the times noted. In the case of the large mesh (48) even saturation failed to prevent penetration, the mosquitoes flying through. Laid on the arm, however, this did give protection as shown, in that only two bites were received 8 days after impregnation, whilst when at a distance from the arm 43 bites were received.

Briefly netting with mesh up to $2-3 \mathrm{~cm}$. diameter

Table 11

\section{Fabric}

Cloth A, a thick hard drill

Cloth B, a thick soft drill

Cloth C, a thin close wove fabric

Cloth $\mathrm{D}$, similar to $\mathrm{C}$, but dyed green

Fabric 84, cotton muslin

Fabric 85, drill

Fabric 88, cambric

$\begin{array}{cc}\begin{array}{c}\text { Weight } \\ \text { of fabric/ }\end{array} & \begin{array}{c}\text { Amount } \\ \text { of fluid } \\ \text { retained in }\end{array} \\ \text { sq.ft. } & \text { cu.mm./ } \\ \text { in g. } & \text { sq.in. } \\ 28 & 154 \\ 25 & 154 \\ 20 & 77 \\ 21 & 77 \\ - & 94 \\ - & 208 \\ - & 58\end{array}$

Table 12

$\begin{array}{ccc}\begin{array}{c}\text { Amount } \\ \text { of DMP } \\ \text { retained }\end{array} & \begin{array}{c}\text { amount } \\ \text { of DMP } \\ \text { causing } \\ \text { oiliness }\end{array} & \begin{array}{c}\text { Days of } \\ \text { complete } \\ \text { protection }\end{array} \\ 20 & 20 & 18 \\ 20 & 20 & 18 \\ 10 & 10-15 & 18 \\ 10 & 10 & 18 \\ 4 \cdot 7 & - & 4 \\ 10 \cdot 4 & - & 4 \\ 2 \cdot 9 & - & 4\end{array}$

Protection period in days with dosage in cu.mm./sq.in. as under

$\begin{array}{cccc}\text { Netting } & \begin{array}{c}\text { Solvent } \\ \text { retained } \\ \text { cu.mm./sq.in. }\end{array} & \begin{array}{c}\text { DMP when } \\ \text { saturated }\end{array} & \begin{array}{c}\text { Maximum } \\ \text { DMP not } \\ \text { objectionable }\end{array} \\ 40 a & 104 & - & 40 \\ 47 & 96 & 79 & \text { Sat. } \\ 48 & 29 & 22 & \text { Sat. }\end{array}$

\begin{tabular}{|c|c|c|c|c|c|c|c|}
\hline \multicolumn{5}{|c|}{ At distance from arm } & \multicolumn{3}{|c|}{ In contact with arn } \\
\hline Sat. & 50 & 30 & 20 & 15 & Sat. & 50 & 20 \\
\hline 15 & 15 & 5 & - & 5 & - & - & - \\
\hline 13 & 13 & 8 & 0 & - & 13 & 8 & 3 \\
\hline 0 & - & - & - & - & 8 & - & 0 \\
\hline
\end{tabular}

\section{(4) Impregnation of netting}

At the suggestion of Brig. J. A. Sinton tests were made of protection given by impregnated netting. Three types were tested, viz. a light fine netting $(40 a)$ with cotton-like strands and mesh about $5 \mathrm{~mm}$. in diameter, a stout heavy netting (47) with strands the thickness of ordinary twine and mesh about $1 \mathrm{~cm}$. diameter and a large mesh net (48) of fishing net type with strands of the thickness of very fine twine and mesh about $2.5 \mathrm{~cm}$. diameter.

The netting was tested in two ways, viz. (1) loosely stretched above the arm at a distance of a few $\mathrm{cm}$. by the box arrangement previously described, and (2) laid in contact with the skin, care being taken that it was not moved more than could be helped after once being laid on. The results are given in Table 12.

Though there was some delay in penetration in the case of the medium mesh netting (first bite at 4 min.) there was practically no obstruction with the thin small mesh or the large mesh. will take up a relatively large amount of DMP without becoming objectionable and will give considerable protection to passage of mosquitoes. In contact with the skin even very large mesh if saturated may give considerable protection up to a week.

\section{PENETRABILITY OF FABRICS TO MOSQUITO BITES}

\section{(1) Technique}

In testing for penetrability the same test procedure was used as for impregnated fabrics, only that 200 in place of 100 female Aedes (with 200 males) were used, the time of exposure as before being $15 \mathrm{~min}$.

Mosquitoes settled freely on the fabric, hunting and probing where immediate penetration was not secured. The number on the fabric at any one time usually ranged, after the first 30 sec. or so, from 40 to 60 , though as shown by the wheals left where the 
fabric was very easily penetrable the number actually attempting to bite must have been much larger.

As with repellency tests the number of wheals gave the most objective means of assessing penetrability, though the time taken before the first bite was felt also usually gave a good indication. After some experience the following code was used for recording degree of penetrability.

Class I. Impenetrable. No wheals.

Class II. Almost impenetrable. An occasional wheal in some tests.

Class III. Penetrable with considerable difficulty. 2 to 9 wheals.

Class IV. Penetrable with some difficulty. 10 to 20 wheals.

Class V. Easily penetrable. Over 20 wheals.

Of the 91 fabrics tested, 31 were found impenetrable, 7 almost impenetrable, 15 penetrable with considerable difficulty, 12 with some difficulty, and 24 easily penetrable. The mean number of wheals and the mean time to first bite felt in these classes were as follows :

\begin{tabular}{|c|c|c|c|c|}
\hline Class & $\begin{array}{l}\text { Mean } \\
\text { time to } \\
\text { first bite } \\
\text { in min. }\end{array}$ & Range & $\begin{array}{c}\text { Mean } \\
\text { number } \\
\text { of wheals }\end{array}$ & Range \\
\hline II & $8 \cdot 0$ & 4-10 & $0 \cdot 75$ & 1 \\
\hline III & $7 \cdot 1$ & $3-10$ & $5 \cdot 4$ & 2-9 \\
\hline IV & $4 \cdot 3$ & $1-8$ & $14 \cdot 5$ & $11-20$ \\
\hline$V^{*}$ & $1 \cdot 7$ & $\frac{1}{4}-3$ & $44 \cdot 0$ & $25-124$ \\
\hline
\end{tabular}

* The number of wheals in this class is the number inflicted in $2 \mathrm{~min}$. at which time with one exception (6 min. with 25 wheals) the tests were terminated since it was by then quite obvious to which class the fabric belonged.

For naked eye detection of pinholes, portions of fabric were held up to a window facing the sky, the observer being in shadow. Direct pinholes appear as small bright points of light or when very fine as a 'star-dust' effect.

For demonstration of oblique pinholes the fabric was held in a plane at $45^{\circ}$ to the observer and rotated in its own plane. By comparing the effect when held at right angles and at $45^{\circ}$ the relative importance of direct and oblique pinholes can be assessed. Stretching the fabric may show pinholes when not otherwise present. Oblique pinholes are usually best seen when the fabric has been rotated to a particular angle, in drills usually when the ribs of the fabric are viewed at an angle of $45^{\circ}$.

For examination under the microscope, preferably a low-power binocular, a portion of fabric is viewed by transmitted light, the hand or other object being used to shade the upper surface. Pinholes at once stand out sharply. If the upper surface of the fabric is not shaded they may not be visible. A rough indication of size of pinholes can be given by measuring their diameter with the aid of an eyepiece micrometer. For an estimate of their number a small glass plate a few centimetres square on which has been pasted paper with a square hole with sides of $\frac{1}{2} \mathrm{~cm}$. square has been found useful.

For counting the weft and warp threads a small piece of fabric a few centimetres square cut with sharp scissors in the direction of the threads is convenient to use. After removing a few threads from the margins by picking these out with fine forceps it is usually obvious which is the weft as the ends of the threads project conspicuously with gaps between them, whereas in most fabrics of the kind examined the ends of the warp threads usually make a continuous fluffy edge. Or should there be a portion of selvedge the weft threads run at right angles to this. Having exposed the projecting cut ends of the weft threads their number to the centimetre can be counted by laying a scale over the fabric close to the edge. Warp threads can most conveniently be counted by placing a scale across the fabric, or if direct counting is difficult by picking out threads to a measured mark.

Measurement of thickness of thread is technically given as the 'count' (see p. 231). As, however,- in the present case technical methods were not open to the writer measurement has been made with the eyepiece micrometer. As with size of pinhole the results are admittedly very rough since there are obvious difficulties in direct measurement of a thread composed of loosely compacted fibres. Further the threads in a fabric often vary greatly in thickness and even the same thread may vary in different places. Nevertheless, the taking of such measurements, though of no value for precision purposes, will be helpful in describing certain features of fabric structure relating to penetrability as dealt with later.

Thickness of the fabric itself has been measured by use of a flat bladed gauge with a vernier scale. Though this is not the technical method of assessing thickness it will serve for present requirements.

\section{(2) Structural features of fabrics in relation to penetrability}

Certain structural features which have relation to penetrability of the 91 fabrics tested are given in Table 16. These features include thickness of fabric, type of weave, number per centimetre and thickness of weft and warp threads, presence and - character of pinholes and the class to which the fabric has been allocated after testing against Aedes aegypti. 
Thickness of fabric. Thickness of the 91 fabrics varied from $0 \cdot 1$ to $0.6 \mathrm{~mm}$. The following tabulation shows relation of thickness of fabric to the classes of penetrability.

Table 13

Thickness in millimetres

$\begin{array}{crrrrrr}\text { Class } & 0 \cdot 6 & 0 \cdot 5 & 0 \cdot 4 & 0 \cdot 3 & 0 \cdot 2 & 0 \cdot 1 \\ \text { I } & - & 2 & 5 & 12 & 12 & - \\ \text { II } & 1 & 3 & 2 & 1 & 2 & - \\ \text { III } & 1 & 2 & 4 & 2 & 6 & - \\ \text { IV } & - & 1 & 7 & 5 & 1 & 1 \\ \text { V } & 1 & - & 3 & 9 & 7 & 1 \\ \text { Totals } & 3 & 8 & 21 & 29 & 28 & 2\end{array}$

It will be clear from this analysis that in the type of fabric dealt with thickness has very little relation to penetrability. For reasons given later it is probable that a fabric would require to be at least $1.0 \mathrm{~mm}$. thick before its thickness alone would render it impenetrable.

Pinholes. That penetrability of fabrics to mosquito bites is largely, if not entirely, dependent on the existence of actual apertures in the structure of the fabric, here called pinholes, is very clearly shown in the following analysis of results (Table 14). warp) may be in contact without any gap. In such a case there may be no direct pinholes, but at points where the warp threads pass through the gap in the weft threads there may still be oblique pinholes. Or very frequently both oblique and direct pinholes are present.

The relation of direct pinholes to fabric structure is simple. It may be illustrated by reference to data in Table 16. Thus in item 1 (a Shirley cloth) since there are 22 weft threads, each measuring $0 \cdot 3 \times 22$ or altogether $0.66 \mathrm{~cm}$. leaving $0.34 \mathrm{~cm}$. unoccupied, and since there are as many gaps as threads each gap will on the average measure $0 \cdot 34 / 22$ or $0.15 \mathrm{~mm}$. Taking now the figures for the warp threads we find as occupied by the threads $0.26 \mathrm{~mm}$. by 48 or $1.24 \mathrm{~cm}$., i.e. there is no space left for gaps. That there is in this case even an excess is due to compression of the warp threads making them bulge where they pass over the weft threads. We may assume, which we shall find to be correct, that there are no direct pinholes.*

Oblique pinholes have a less obvious derivation. They occur for the most part where a warp thread dipping down to pass through the gap in the weft thereby exposes a view into the weft gap passed over by the succeeding warp thread. Thus they are

Table 14

\begin{tabular}{|c|c|c|c|c|c|c|}
\hline $\begin{array}{l}\text { Class } \\
\text { of } \\
\text { fabric }\end{array}$ & $\begin{array}{l}\text { No. } \\
\text { tested }\end{array}$ & $\begin{array}{c}\% \\
\text { with } \\
\text { pinholes }\end{array}$ & $\begin{array}{c}\text { Average } \\
\text { diameter } \\
\text { of pinholes* }\end{array}$ & $\begin{array}{l}\text { Maximum } \\
\text { size of } \\
\text { pinholes* }\end{array}$ & $\begin{array}{l}\text { Average } \\
\text { number of } \\
\text { pinholes } \\
\text { per } \mathrm{cm} .^{2}\end{array}$ & \\
\hline I & 33 & 45 & $0 \cdot 01$ & $0 \cdot 02$ & 172 & In one case slit-like $0.05 \times 0.01 \mathrm{~mm}$. \\
\hline II & 7 & 86 & 0.02 & 0.05 & 102 & - \\
\hline III & 15 & 100 & 0.06 & $0 \cdot 1$ & 129 & One slit-like $0.25 \times 0.01 \mathrm{~mm}$. \\
\hline IV & 12 & 100 & $0 \cdot 1$ & $0 \cdot 11$ & 305 & At nearly every intersection \\
\hline V & 24 & 100 & $0 \cdot 11$ & $0 \cdot 4$ & 824 & - \\
\hline
\end{tabular}

From this table it can be seen that fabrics may be impenetrable even when pinholes are numerous if they do not exceed about $0.02 \mathrm{~mm}$. in diameter. They are practically impenetrable even if numerous pinholes exist (often several hundreds to the $\mathrm{cm} .{ }^{2}$ ) up to $0.05 \mathrm{~mm}$. in diameter. Where pinholes reach or exceed $0.1 \mathrm{~mm}$. in diameter penetration takes place with more or less ease.

Fabric structure. The number and size of pinholes is intimately connected with fabric structure. Fabrics consist of two series of threads crossing one another at right angles, viz. the warp threads which are woven into and the weft threads which are woven in. Both warp and weft threads may be spaced with an interval or gap between consecutive threads, in which case where a gap in one series coincides with a gap in the other there is formed a perforation through the fabric, i.e. a direct pinhole.

In other cases the threads of one series (usually the especially likely to occur in $3 / 1$ weave (as in drills) where the warp thread passes over three consecutive weft threads before entering a gap in the weft. A wide weft gap also favours such exposure. It is practically impossible to measure the size or even to count oblique pinholes, one reason being that it is

* In general, if there are $x$ threads of $y$ thickness to the $\mathrm{cm}$. (10 mm.) then the average gap, $g$, should be

$$
g=\frac{10-x y}{x} .
$$

This value $x y$ is largely equivalent to the 'cover factor' of technical terminology, though not identical since technically thickness of thread is deduced from weight of a skein of known length of thread. If $x y$ is equal to or greater than 10 the threads are touching or compressed together. If $x y$ equals 5 , the gaps and threads are of equal extent (see also note by kindness of $\mathrm{Mr}$ Whinfield given in Appendix C). 
not so much the actual visible pinhole which decides whether entry can be made as the potential entry into the wide weft gap which the structure permits. This will be readily appreciated if in some soft drill under the binocular one of the warp threads as it passes over the three weft threads is gently pushed aside with the needle at the point where the next thread passes into a gap. The size of the opening so disclosed is very unexpected and is no doubt the reason why even seemingly stout and thick drills are usually penetrable.

\section{(3) Type of fabric}

An abstract of the results obtained with the 91 fabrics in relation to type of fabric is given in Table 15. More complete details will be found in Table 16.
Twenty-four drills have been tested. Some of these were soft pleasant fabrics, though in this case usually penetrable. Those that were impenetrable tended to be stout, rather thick and firm. Weft threads very commonly number about 20 and the warp threads 40 per $\mathrm{cm}$., and measure respectively (by micrometer) 0.35 and $0.25 \mathrm{~mm}$. Weave is $3 / 1$ and both oblique and direct pinholes are common. The drills are a popular fabric for uniform purposes, but if sufficiently loosely woven to be pleasantly soft they seem almost certain to be penetrable and often surprisingly so.

The remaining fabrics were of a miscellaneous character. Their chief features in relation to penetrability are shown in Table 16. Fabrics with $2 / 1$ and $2 / 2$ weave were often relatively mosquito proof since they have not the vulnerability of the $1 / 1$ woven

Table 15

\begin{tabular}{lrrrrrr}
\multicolumn{7}{c}{ Classes } \\
$\quad$ & $\overbrace{\text { Fabric }}^{\text {I }}$ & II & III & IV & V & Total \\
Shirley cloth & 10 & - & - & - & - & 10 \\
Poplins* & 13 & 2 & 4 & 1 & 4 & 24 \\
Drills & 3 & 2 & 4 & 10 & 5 & 24 \\
Bluettes & 3 & - & 1 & - & - & 4 \\
Shirt & 3 & - & 1 & 1 & 1 & 6 \\
Calico & - & - & - & - & 3 & 3 \\
Cambric & - & - & 1 & - & 2 & 3 \\
Muslin & - & - & - & - & 1 & 1 \\
Others & 1 & 3 & 4 & - & 8 & 16
\end{tabular}

* Most of these were specially woven to be mosquito proof.

The only type of fabric which has invariably been impenetrable is the Shirley cloth type (Oxford weave). These are closely woven fabrics with $1 / 1$ weave in which the warp threads are double, each element and the weft threads being of approximately the same thickness. The tightly packed and bulged double warp gives such fabrics under the microscope an extremely characteristic appearance. Such fabrics have usually been about $0.3 \mathrm{~mm}$. thickness by gauge, but vary with thickness of the yarn used, some being as thin as the poplins $(0.2 \mathrm{~mm}$.). They are apt to be rather 'hard' but the thinner ones may be quite soft.

Next to the Shirley cloths in imperviousness to mosquito bites were certain poplins which had been specially woven to make them mosquito proof. They have all been soft, thin, pleasant, silky fabrics. Weft and warp threads are usually approximately equal with $1 / 1$ weave. A series of samples has been tested with the warp numbering 63 threads to the centimetre. Where the weft threads numbered 30-33 they were impermeable. Those samples with weft numbering 26-29 were readily penetrable. Pinholes are usually slit-like and at a certain critical point become penetrable. fabrics and have insufficient length of slack in the warp threads to favour oblique pinholes. Simple 1/1 weave without special precautions to ensure nonpenetrability and as used in such fabrics as calico, cambric, etc. usually gave little or no protection. Fabrics of the cellular type such as Aertex and cellular bush shirts had no protective value.

In general and considered in relation to the severity of the test employed classes I, II and even III might be considered to be highly protective if not completely impervious to bites. Classes IV and V are unsuited for the purpose of affording protection.

\section{(4) Mechanism of penetration}

In view of the results recorded some interest attaches to the mechanism by which such fabrics are penetrated.

The mouth parts of the mosquito consist of an outer scaled sheath, the proboscis (labium), within which is enclosed a number of fine stylets held together by lubricating fluid to form a smooth delicate naked organ, the fascicle. The labium of Aedes aegypti is about $2 \mathrm{~mm}$. long by $0.11 \mathrm{~mm}$. in diameter. The fascicle is much narrower being only $0.03 \mathrm{~mm}$. in diameter. 
Table 16

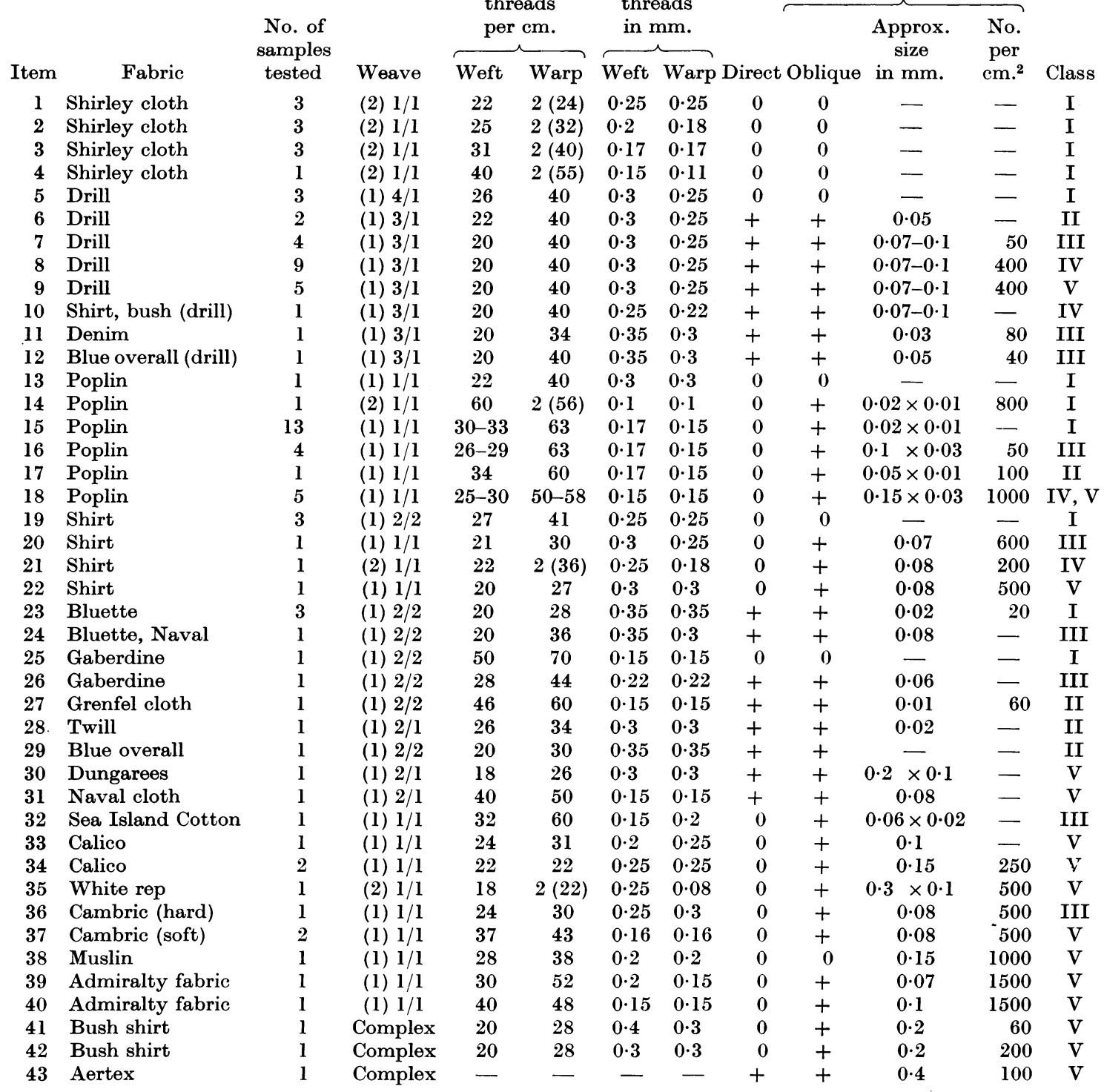

Only the fascicle is inserted into the tissues, the tip of the labium or visible proboscis being merely placed on the skin surface, where by means of the two flap-like labella it supports and guides the fascicle much like a billiard player guides his cue. As the fascicle sinks into the skin the labium, which is not a tube but a deeply grooved sheath, buckles and thereby the fascicle is forced out of the groove except at its origin and where the labella still retain their hold. At full penetration the labium is bent back under the neck in a sharp U-shaped curve, the fascicle being thereby permitted to penetrate for the greater part of its length. It is obvious that unless the labium is able to bend back in this way the fascicle cannot be fully, if at all, extruded. How far it could penetrate without the labium being obviously buckled is problematical, but at the best it could be only a small fraction of its length.

The question now arises as to what happens when a mosquito bites, or attempts to bite, through a fabric. If it can insert the whole proboscis through the fabric, reach the skin, find room to bend back 
the labium, and bring the head near enough to the skin surface to allow the fascicle to enter sufficiently deeply into the tissues to tap blood, then it can feed in the normal manner. This is undoubtedly what usually happens when a fabric is penetrated. The mosquito hunts and probes until it finds a pinhole into which it can insert the whole proboscis.

These facts are sufficient to account for most of the results obtained. Since no fabric has been submitted as likely to be used for tropical uniform with a greater thickness than $0.6 \mathrm{~mm}$., it is obvious that thickness alone in this case does not ensure protection. It is of interest, however, to note how small a gap between fabric and skin is necessary to eliminate biting, probably not much more than a millimetre, allowing for thickness of the fabric and some passage of the fascicle into the tissues. This explains too what experience has shown, viz. that mosquitoes rarely bite if there are two layers of clothing. It also explains how use of a light netting below a single layer of clothing may prevent biting. Further it explains how even very thick loose fabrics, such as woollen socks, offer little or no protection in a single layer but may be fully protective if double.

That insertion of the proboscis as a whole is the usual method of penetration of fabrics of the type tested is clear from the abrupt transition from impenetrability to penetrability and the correlation of this with existence of pinholes approaching or exceeding $0.1 \mathrm{~mm}$. diameter. Those fabrics which are 'almost impenetrable' or 'penetrable with consider: able difficulty' are most probably penetrated not through such pinholes as may have been noted on microscopic examination, but by occasional larger pinholes due to some slight defect in the fabric. In a fabric such as item 7 in the table there would be some 250 pinholes $/ \mathrm{sq} . \mathrm{cm}$. and therefore over the exposed area of fabric some 47,000 such, but only one bite was received in $15 \mathrm{~min}$. with mosquitoes averaging 5 or 6 to the square inch in some parts. It is clear that pinholes of the size present, $0.05 \mathrm{~mm}$., did not permit of penetration. Even class IV (not more than 20 bites) must be considered by no means offering a large number of openings suitable for penetration. It is not until one is dealing with class $V$ that there is clearly a free field to penetration and here pinholes are almost invariably numerous up to $0.1 \mathrm{~mm}$. diameter or more. In fabrics of ordinary thickness it is then possible to say that biting is only possible by insertion of the whole proboscis and that the fascicle is not used to penetrate openings too small for the proboscis.

It is still necessary to consider the case of very thin fabrics. One such is item 16, a thin loosely woven poplin (thickness $0 \cdot 1 \mathrm{~mm}$.), with small slitlike openings $0.02 \times 0.1 \mathrm{~mm}$. ( 800 per $\left.\mathrm{cm} .{ }^{2}\right)$ which was nevertheless impenetrable. So loose and soft were the threads that it required only a touch with the

J. Hygiene 45 needle to open the slits amply wide enough for passage of the fascicle. Yet no such use was evidently made of this organ. Three similar thin and soft poplins (thickness $0.2 \mathrm{~mm}$.) received only 2,5 and 9 bites respectively. It would seem, therefore, that even very thin and soft fabrics are not penetrated in the absence of pinholes and that the fascicle is not commonly used alone to penetrate.

There remains the question of what is the smallest sized pinhole that will admit the proboscis. Something must here be allowed for differences in the firmness of threads limiting the pinholes and rigidity or the reverse of the stray cotton fibres which are usually seen encroaching upon the lumen of such. But in direct pinholes the limit would appear to be in the neighbourhood of $0.07 \mathrm{~mm}$., for squarish or round pinholes. Slit-like pinholes apparently require to be of the order of $0.15 \times 0.03 \mathrm{~mm}$., i.e. that both length and breadth are factors. In both cases it would appear that the proboscis can exert a certain, though small, degree of pressure in pushing aside the threads to enlarge the pinholes. In item 39, a cambric which appeared to have been smoothed on one surface and was noticeably rather 'hard', only 8 bites were received, though pinholes up to 0.08 were present at almost every intersection. The thickness of the fabric was only $0 \cdot 2 \mathrm{~mm}$.

The main controlling factor in oblique pinholes would seem to be looseness of the warp threads, especially in a $3 / 1$ weave fabric, with wide weft gap.

\section{SUMMARY AND CONCLUSIONS}

1. A general survey has been given of the subject of repellents against mosquitoes. Results recorded in published literature prior to the war have been summarized and those from the greatly increased volume of unpublished research during the war briefly outlined.

2. Of the older literature it has been shown that results were often conflicting due to many of the substances tested not being pure chemicals but very commonly essential oils and other products having a variable composition and to the methods used in testing being insufficiently precise and standardized. Particularly, standardization was lacking in that most important respect, viz. the condition of the mosquitoes used in the tests. The whole treatment of repellency on a scientific basis depends upon the suitability of the methods used in testing and for this reason a full and detailed account has been given in $\S I V$ of the methods used in the present enquiry in rearing standardized cultures of Aedes aegypti and in $\S \mathrm{V}$ of the methods of laboratory testing and of interpreting results.

3. Some remarks have been made on the proper sphere of field tests. Field tests, it is pointed out, are extremely valuable but less suitable than laboratory 
tests for determining with precision the properties of repellent substances owing to the impossibility of obtaining controlled conditions. The proper sphere of such tests would appear to be as an extension of the results of laboratory tests to cover the many qualifying circumstances which are introduced when repellents of known effectiveness are used in practice.

4. A description has been given in § III of the chief features of such repellents as have been in use, and in $\S \mathrm{VI}$ are given the results of testing a considerable number of compounds and preparations, with data showing their comparative repellent power and duration of protection afforded. In this series three types of compound especially exhibit repellency, viz. (1) unsaturated long-chain alcohols, aldehydes and phenolic compounds such as are characteristic of most essential oils, oil of citronella, due to its active principle citronellal, being one of this type; (2) high boiling-point esters of the lower methyl series with various high molecular weight acids, such as phthalic, adipic, cinnamic, citric, etc., of which dimethyl phthalate is the best-known example, and (3) high boiling-point alcohols, especially diols, which need not necessarily be unsaturated or contain methyl series groups, of which Rutger's 612 or 2ethylhexane-1:3-diol is that best known. There are, however, undoubtedly many other groups which for some reason may possess this property as may be instanced the last entry on the list of compounds tested which belongs to none of these groups but was found as powerful as any compound tested.

5. Attention has been directed to the importance, in connexion with duration of effect, of the boilingpoint. It is shown that to give protection for a period sufficient to qualify a substance to be considered a repellent, i.e. in the criterion adopted by the Unit a protection period of $2 \mathrm{hr}$., it must have the relatively high boiling-point of at least $250^{\circ} \mathrm{C}$. at $760 \mathrm{~mm}$. pressure. Compounds with lower boilingpoint merely volatilize completely on the warm skin by the time this period is reached. To give protection for $6 \mathrm{hr}$. the boiling-point must be at least of the order of $280^{\circ} \mathrm{C}$. Substances with boiling-point much over this, whilst they show lasting effect, are apt to exhibit a lower grade of repellency. In this connexion a method, sufficiently accurate for the purpose, has been quoted by which any boiling-point given as at a reduced pressure can be reduced to its theoretical boiling-point at $760 \mathrm{~mm}$. The basis on which the necessary factors for this have been arrived at will be found in a note kindly furnished by Dr E. A. Moelwyn-Hughes given as an appendix.

6. The question of rub-resistant creams, of measurement of effect of repellency at a distance, of impregnation of clothing with repellents and of the testing of penetrability of clothing fabrics to mosquito bites are dealt with in their respective sections and any conclusions regarding these subjects there given. The last has been dealt with in some detail since no account of such testing has, so far as is known, been given in the literature. A note by Mr J. R. Whinfield, Ministry of Supply, giving technical methods of measuring structural features of fabrics has been given as an appendix and should be useful to anyone taking up the study of fabrics from this point of view.

7. It remains desirable, perhaps, to indicate in a general way what recent advances in regard to mosquito repellents amount to in practice, apart altogether from theoretical or scientific considerations. Results during the war have related almost entirely to the use of repellents for military purposes and the repellent almost universally employed in practice has been dimethyl phthalate. A number of reasons have combined to make this the repellent of choice for such a purpose. For the American Army the so-called 6:2:2 mixture, i.e. a mixture in the above proportions of dimethyl phthalate, Rutger's 612 and indalone, has been the official repellent. But Rutger's 612 has never been so freely available as dimethyl phthalate and neither it nor indalone has been in extensive use in this country. Dimethyl phthalate has very generally been found most suitably used 'straight' being carried in a suitable receptacle from which a little is shaken out into the palm of the hand and used to anoint exposed skin areas or even clothing. For impregnation of tropical uniforms it has usually been used suitably dissolved in a solvent or as an emulsion and the articles sprayed or dipped and wrung out. It is effective against many forms of biting insects and has been used against mites. In this last respect, however, it has been to some extent replaced by dibutyl phthalate, which when used for impregnating clothing is by reason of its higher boilingpoint more resistant to washing.

8. For civilian use there would appear to be a wider field. One paramount requirement for military use has always been that the repellent should give very prolonged protection, i.e. that it should retain its effects for such periods as overnight, or as often specified for $6 \mathrm{hr}$. This has limited the choice of repellents excluding many substances which might be very effective for shorter periods. For the civilian such emphasis on duration of effect does not arise, for circumstances must be rare in which the application could not be renewed, say after $2 \mathrm{hr}$. if necessary. Again should it only be a matter of giving comfort against moderate numbers of mosquitoes something less drastic than anointing all bare skin with dimethyl phthalate might well suffice. For military purposes smell is a serious, indeed fatal, drawback. For the civilian a pleasant smell might even be an advantage. The civilian too would probably prefer a light pleasant cream to the 
repellent in liquid form. There is much scope here for the chemist and the cosmetician.

What then at present should the civilian be advised to use? This is dependent very largely on what he can now readily purchase, and there is some difficulty in saying under present circumstances what this might be. If a repellent is required to prevent attack where mosquitoes are a serious nuisance, and the same applies to midges, probably the best advice would be to try dimethyl phthalate straight as in military use. This might be obtained by order through a chemist to some wholesale firm such as Boake Roberts and Co. or Stafford Allan and Co. These or other firms would probably supply a moderate quantity, say $4 \mathrm{oz}$. on such an order. This could be used as stock from which to fill a small corked bottle for use. A corked bottle is better than a stoppered one as dimethyl phthalate is very liable to leak. It should be applied by pouring a little in the palm of the hand, rubbing the hands together and anointing any exposed parts such as the neck, behind the ears and even the face, but avoiding neighbourhood of the eyes. It might be more pleasant, especially if conditions were not so severe, to use a dimethyl phthalate cream such as Dr Hamil's very pleasant wax and arachis oil cream or the $70 \%$ magnesium stearate Porton cream as given in $\S \mathrm{VI}(2)$. This could probably be made up to the formula given by a dispensing chemist. If it were possible to obtain it, a good Java citronella oil would probably be even better, for the great advantage here is that a good citronella oil will keep mosquitoes at a distance and complete covering of skin with the repellent is not so necessary as with dimethyl phthalate. Or if such an oil is not to be at present obtained it might be possible to get citronellal, its active principle, or even citronellol the corresponding alcohol. A little experimentation and careful preliminary trial is always desirable as to what can be done and as to how effective the substance seems to be. This applies especially to the case of midges which are not always the same species and might not in some cases be repelled. It should be realized that for civilian use there is still much that is experimental. For those with a liking for experiment it might be well worth while to try out that very pleasant smelling substance hydroxycitronellal, a note on which will be found in § III.

Mention should also be made of certain proprietary products issued by certain American firms which might at any time be obtainable in this country, such as 'Skat' or 'Eveready repellent'. Skat (Skol Company Inc., New York) is issued in two forms, viz. as containing dimethyl phthalate and as containing indalone (in both cases practically these substances), and Eveready Insecticide (National Carbon Co. Inc., New York) as 'formula 612', in this case Rutger's 612. Very likely in time good repellents will eventually be put on the market by various firms in this country.

9. As regards the future no limit can probably be placed upon what may result from organized research which is likely to be taken up more seriously now than was previously thought worth while. Research in this field may be said to have reached a point where methods of testing will enable precise results to be obtained and if work by the synthetic chemist is linked with that of the insect biologist further progress in the discovery of still more effective repellents is extremely likely. Research should not, however, stop here for much still remains to be done in the direction of ascertaining methods of using such repellents to the best advantage to meet different requirements and even of ascertaining exactly what such requirements are.

\section{ACKNOWLEDGEMENTS}

Very little could have been done by the Unit if it had not received the constant interest and help which it did from the Office of the Scientific Advisers, Ministry of Production. In this respect besides Sir Ian Heilbron who initiated the Unit I wish to thank Dr A. King, Dr F. N. Woodward, Dr A. F. Millidge and Mr F. Barlow, Imperial College, for the unstinted interest and help they gave in sending for test interesting synthetic compounds and in other ways, also Dr R. A. E. Galley, Agricultural Research Council, for his interest in the work and its circulation in duplicated form prior to publication. A grant by the Medical Research Council towards the working of the Unit is also gratefully acknowledged. I must also thank Major-General D. T. Richardson, Prof. P. A. Buxton, Dr V. B. Wigglesworth and Dr E. A. Carmichael for much courtesy and help in various ways. To Dr A. D. Imms, in whose laboratory the Unit has worked, I am deeply indebted for much kind help and consideration at all times.

I also wish to acknowledge the very great help given by $\mathrm{Mr} \mathrm{G}$. W. Otter, whose services were lent to the Unit during 1944 by the Ministry of Food (Infestation Branch) and whose ingenuity, skill and helpful interest in the work added greatly to the success of the Unit, as also the very able services of Mr J. A. Musgrave who assisted me during 1945 and to whom I am indebted for much kind help during that year. 


\section{LIST OF WORKS DEALING WITH MOSQUITO REPELLENCY}

\section{A. References to repellency}

Bacot, A. W. \& Talbot, G. (1919). The comparative effectiveness of certain culicifuges under laboratory conditions. Parasitology, 11, 221-36.

Bunker, C. W. O. \& Hirschfelder, A. D. (1925). Mosquito repellents. Amer. Jour. Trop. Med. 5, 359-83.

Calvary, H. O. (1945). (On toxicity tests.) Food Packer, April 1945.

Christophers, S. R. (1945). Insect repellents. Brit. Med. Bull. 3, 222-4.

Coogle, C. P. (1923). A preliminary report on the use of creosote oil as a mosquito repellent. Publ. Hlth Rep., Wash., 38, 437-43.

Coogle, C. P. (1923). A preliminary report on the use of creosote oil as a mosquito repellent. Publ. Hlth Bull., Wash., no. 137, 145-56.

CoogLe, C. P. (1925). Mosquito repellents and mosquitocides. Publ. Hlth Bull., Wash., no. 156, 136-7.

Cooley, R. A. et al. (1928). Work done by the Entomological Department. Control of mosquitoes. 34th Ann. Rep. Montana Agric. Exp. Sta. 1926-7, pp. 90-3.

Covell, G. (1941). Malaria Control by Antimosquito Measures, 2nd ed. London: W. Thacker and Co.

Dethier, V. G. (1943). Testing attractants and repellents. Publ. No. 20. Amer. Ass. Adv. Sci. pp. 167-72.

Dover, C. (1930). An improved citronella mosquito deterrent. Indian J. Med. Res. 17, 961.

Drarze, J. H. et al. (1934). (On toxicity tests.) J. Pharmacol. 82, 159.

Draize, J. H. et al. (1944). (On toxicity tests.) Chem. Engng. News, 22, 1503.

DunN, M. B. (1925). Methods of protection from mosquitoes, etc. Canada Dep. Agric. Pamphlet, n.s. no. 55 .

Echstein, F. (1920). Aus einer Feldstation fur Stechmucken. Z. angew. Ent. 6, 338-71.

EdTorial (1934). Essential oils from East Africa. II. Grass oils. Bull. Imp. Inst. 32, 216, 263-5.

Edtorial (1942). (Thanite.) Science, 95, no. 2473, Supplement, p. 10.

Fletcher, T. B. (1920). Report of the Imperial Pathological Entomologist. Sci. Rep. Agric. Res. Inst. Pusa, 1919-20, pp. 95-108.

Fowler, C. E. P. (1926). Malaria in Palestine. J. $R$. Army Med. Cps, 46, 264.

Freeborn, S. B. (1928). Observations on the control of Sierran Aedes (Culicidae, Diptera). Pan Pacific Cong. Ent. 4, no. 4, 177-81. Abs. Rev. App. Ent., Med. and Vet. 16, 214.

Gibson, A. \& Twinn, C. R. (1939). Household insects and their control. Publ. Dep. Agric. Canada, no. 642 (Ent. Bull. no. 30), Ottawa, 100 pp.

Ginsburg, J. H. (1935). Protection from mosquito bites in outdoor gatherings. Science, 82, 490-1.

Ginsburgh, J. M. (1944). Mosquitó oils, larvicides, repellents, outdoor sprays and their application. Bull. N. Jersey Agric. Exp. Sta. no. 711, 12 pp.
Given, D. H. C. (1922). The campaign against mosquitoes on board H.M.S. Cadmus in 1912 (Citronella). J. R. Naval Med. Serv. 8, 265-9.

Granett, P. (1938). Comparison of mosquito repellency tests under laboratory and field conditions. Proc. 25 th Ann. Meet. N. Jersey Mosq. Exterm. Ass. pp. 51-7.

Granett, P. (1940). The development of a practical mosquito repellent. Proc. 27th Ann. Meet. N. Jersey Mosq. Exterm. Ass.

GranetT, P. (1940). Studies of mosquito repellents. I. Test procedure and method of evaluating test data. J. Econ. Ent. 33, 563-5.

GranetT, P. (1940). Studies of mosquito repellents. II. Relative performance of certain chemicals and commercially available mixtures as mosquito repellents. J. Econ. Ent. 33, 566-72.

Harry, R. G. (1941). Modern Cosmetology. London: Chapman and Hall.

HoFFMAN \& Gildmeister (1913-16). The volatile oils, 2nd ed.

Howard, L. O. (1917). Remedies and preventatives against mosquitoes. Frmrs' Bull. U.S. Dep. Agric. no. 444,15 pp.

Howard, L. O. \& Bishopp, F. C. (1928). Remedies and preventatives against mosquitoes. Fmrs' Bull. U.S. Dep. Agric. no. 1570.

ImPERIAL InSTITUTE (1940). A Survey of Insecticidal Materials of Vegetable Origin. London: Imperial Institute.

JoACHIM, A. W. R. (1937). The present position of the trade in Ceylon citronella oil. Trop. Agriculturalist, 88, 108-11.

Knipling, E. F. \& Dove, W. E. (1944). Recent investigations of insecticides and repellents for the Armed Forces. J. Econ. Ent. 37, 477-80.

MaCNAy, C. G. (1938). An effective repellent for biting insects. Canadian Ent. 70, 175-6.

Macnay, C. G. (1939). Studies on repellents for biting flies. Canadian Ent. 71, 38-44.

Madden, A. H., Lindquist, A. W. \& Knipling, E. F. (1943). Tests of repellents against chiggers (Acariseus and Eutrombicula). J. Econ. Ent. 37, 283-6.

MaIL, G. A. (1934). The mosquitoes of Montana. Bull. Montana Agric. Exp. Sta. no. 288, pp. 64-70.

Marriott, W. (1911). Hints to Meteorological Observers, 7th ed. London: Stanford.

Marvin, C. F. (1941). Psychrometric Tables. Washington: U.S. Dep. Commerce, Government Printing Office. (Obtainable in this country from C. F. Casella and Co. Ltd., Regent House, Fitzroy Square, London, W. 1.)

Merllon, B. DE (1934). Observations on Anopheles funestus and Anopheles gambiae in the Transvaal. Publ. S. Afr. Inst. Med. Res. 248 pp. (repellents, pp. 238-40).

Moore, W. (1934). Esters as repellents. J. N.Y. Ent. Soc. 42, 185-92.

Nelson, A. A. et al. (1944). (Toxicity tests.) Publ. Hlth Rep. 59, 1009. 
Nelson, E. K. \& Russell, G. A. (1925). Essential oils. In Allen's Commercial Organic Analysis, 5th ed. 4, 1-217.

Nernst, W. (1923). Theoretical Chemistry. London. (Very complete account of boiling-point and other physical characters.)

PARry, E. J. (1922). The Chemistry of Essential Oils and Artificial Perfumes, 4th ed.

Patterson, T. C. (1940). Insect pests in northern Norway. The mosquito nuisance. J. R. Nav. Med. Serv. 26, 346-52.

Plumb, G. H. (1944). Lethane 384 special for control of the brown dog tick. J. Econ. Ent. 37, 292-3.

Roy, D. N. \& GHosh, S. H. (1942). Further work on the comparative efficacy of different culicifuges under laboratory conditions. Parasitology, 34, 291-4.

Roy, D. N., Ghosh, S. H. \& Chopra, R. N. (1942). Comparative efficacy of different culicifuges under laboratory conditions. Parasitology, 34, 152-4.

RUdolfs, W. (1922). Chemotropism of mosquitoes. Bull. N.J. Agric. Exp. Sta. no. 367.

Rudolfs, W. (1923). Observations on the relation between atmospheric conditions and behaviour of mosquitoes. Bull. N.J. Agric. Exp. Sta. no. 388.

Rudolfs, W. (1924). Influence of external conditions upon the behaviour of mosquitoes. Proc. 11th Meet. N.J. Mosq. Exterm. Soc. pp. 58-64.

Rudolfs, W. (1925). Relation between temperature, humidity and activity of house mosquitoes. J. N.Y. Ent. Soc. 33, 163-9.

Rudolfs, W. (1925). Studies of mosquito repellents. Rep. N.J. Agric. Exp. Sta. 1923-4, pp. 350-3.

RuDolfs, W. (1926). Investigations of mosquito problems. Proc. 13th Ann. Meet. N.J. Mosq. Exterm. Soc. pp. 33-54.

Rudolfs, W. (1930). Effects of chemicals upon the behaviour of mosquitoes. Bull. N.J. Agric. Exp. Sta. no. 496 .

Shaw, A. O., Smith, R. C., Atkeson, F. W. et al. (1943). Tests of repellents of known ingredients and of selected commercial sprays on dairy cattle. J.Econ. Ent. 36, 13-32.

Sмrтн, J. B. (1901). Report of the Entomological Dep., N.J. Agric. Coll. Exp. Sta. p. 542 (quoted by Granett, 1940).

SyddIQ, M. M. (1938). Observations of practical importance for the malariologist. Indian Med. Gaz. 73, 676-9.

Woodard, G. W. et al. (1944). (Toxicity tests.) $J$. Pharmacol. 82, 152.
Yothers, M. A. \& Carlson, F. W. (1944). Repellency of pyrethrum extract and other materials to full-grown codling moth larvae. J. Econ. Ent. 37, 617 .

\section{B. References having relation to laboratory use of Aedes aegypti}

Atkin, E. \& Bacot, A. (1917). The relation between the hatching of the eggs and the development of the larvae of Stegomyia fasciata and the presence of bacteria and yeasts. Parasitology, 9, 482-536.

Buxton, P. A. \& Hopkins, G. H. E. (1927). Researches in Polynesia and Melanesia. Parts I-IV. Mem. Lond. Sch. Hyg. Trop. Med. no. 1.

Connell, W. A. (1941). Hatching response of Aedes sollicitans eggs under selected and controlled environmental conditions. J. Econ. Ent. 34, 187-92.

David, W. A. L., Bracey, P. \& Harvey, A. (1944). Equipment and method in breeding Aedes aegypti $\mathrm{L}$. for the biological assay of insecticides. Bull. Ent. Res. 35, 227-30.

Golberg, L., De Meillon, B. \& Lavoipierre, M. (1945). The nutrition of the larva of Aedes aegypti L. II. Essential water-soluble factors from yeast. J. Exp. Biol. 21, 90-6.

Hinman, E. H. (1930). Amer. J. Hyg. 12, 238.

Howard, L. O. (1913). The yellow fever mosquito. Fmrs' Bull. U.S. Dep. Agric. no. 547, pp. 1-16.

Johnson, H. A. (1937). Notes on the continuous rearing of Aedes aegypti in the laboratory. Publ. Hlth Reps., Wash., 52, 1177-9.

Lewis, D. J. (1933). Observations on Aedes aegypti L. (Diptera, Culicidae) under controlled atmospheric conditions. Bull. Ent. Res. 24, 363-72.

Meillon, B. DE, Golberg, L. \& LavoipierRe, M. (1945). The nutrition of the larva of Aedes aegypti L. I. J. Exp. Biol. 21, 84-9.

Roy, D. N. (1936). On the role of blood in ovulation in Aedes. Bull. Ent. Res. 27, 423-9.

Seaton, D. R. \& Lumsden, W. H. R. (1941). Observations on the effect of age and light on biting of Aedes aegypti (L.) in a controlled microclimate.

Trager, W. (1935). The culture of mosquito larvae free from living organisms. Amer. J. Hyg. 22, 18-25.

Trager, W. (1935). On the nutrition requirements of mosquito larvae (Aedes aegypti). Amer. J. Hyg. 22, 475-93.

Trager, W. (1937). A growth factor required by mosquito larvae, J. Exp. Biol. 14, 240-51. 


\section{APPENDIX A}

\section{STATISTICAL STUDY OF DATA PROVIDED BY 'CONTROLS' AND 'NORMAL FEEDS' 'Controls'}

As previously noted a 'control' exposure has been carried out prior to each test, i.e. an untreated arm with 30 sq.in. of the back of the forearm exposed is inserted for $15 \mathrm{sec}$. into the test cage containing 100 female and 100 male Aedes aegypti aged 5-7 days from emergence, the number of mosquitoes settling and the number of wheals which developed being counted.

Since the data were available it was thought of interest to ascertain how far such figures indicated differences in the settling and biting rate due to season, strain used and subject bitten. An analysis was therefore made of such controls as were carried out during the 12 months May 1944 to April 1945 inclusive relating to two strains (strains $J$ and $L$ ) and two subjects (subjects $\mathbf{A}$ and $\mathbf{B}$ ).

Frequency of settlings and wheals. The following is the total number of settlings arranged in numerical order. The number of observations was 209 and the number of settlings 3390 or a mean of 16.2 mosquitoes per exposure. For wheals the number of observations was 95 and the wheals 1046 or a mean of $11 \cdot 0$.

$\begin{array}{lcccccc}\text { Classes } & 1-5 & 6-10 & 11-15 & 16-20 & 21-25 & 26-30 \\ \text { Settlings } & 0 & 24 & 78 & 78 & 29 & 0 \\ \text { Wheals } & 4 & 46 & 23 & 19 & 1 & 1\end{array}$

The proportion of wheals to settlings cannot be deduced from the above since wheals for one subject have not been recorded for the whole period owing to the development of immunity making these too indefinite for accurate counting. The number of settlings and wheals for subject $B$, however, allows of such comparison if eight observations are omitted where wheals were not recorded. In this case settlings and wheals were:

$\begin{array}{lcccccc}\text { Classes } & 1-5 & 6-10 & 11-15 & 16-20 & 21-25 & 26-30 \\ \text { Settlings } & 1 & 11 & 26 & 19 & 18 & - \\ \text { Wheals } & 1 & 33 & 20 & 19 & 1 & -\end{array}$

The total number of observations in both cases was 74 , the number of settlings 1191 or mean of $16 \cdot 1$ and the number of wheals 886 or mean of $12 \cdot 0$.

The proportion of wheals to settlings was thus $\mathbf{7 4 . 2} \%$. This proportion holds even if the different classes of settlings be considered separately. Thus the percentage for classes 6-10 to 21-25 were respectively $75,71,75$ and $75 \%$.

A word of explanation is perhaps desirable. This does not mean that $25 \%$ of mosquitoes did not attack. As a result of direct observation it had been estimated that it required a mosquito to have been settled 6 sec. before a wheal would be formed. Taking this value those mosquitoes which did not alight on the arm until 6 sec. before conclusion of the observation would not have had time to puncture sufficiently to leave any record in the shape of a wheal. If settling were at a uniform rate during the 15 sec. only $\frac{9}{15}$ or $60 \%$ of mosquitoes would leave wheals, a figure not so very far removed from the $75 \%$ actually found.

Variation due to season, strain and subject. In Table 17 results have been classified according to season, whether strain $J$ or $L$ and whether subject $A$ or B. The somewhat unexpected result was found that no significant difference is shown in any of these respects, the mean settlings, though possibly slightly increased in the August-September period, remaining about $16 \%$ with wheals about $12 \%$.

Table 17

\begin{tabular}{|c|c|c|c|c|c|c|c|c|c|c|c|c|c|}
\hline \multirow[b]{3}{*}{$\begin{array}{c}\text { Exp. } \\
\text { no. }\end{array}$} & \multirow[b]{3}{*}{ Period } & \multicolumn{6}{|c|}{ Strain $J$} & \multicolumn{6}{|c|}{ Strain L } \\
\hline & & \multicolumn{3}{|c|}{ Subject A } & \multicolumn{3}{|c|}{ Subject B } & \multicolumn{3}{|c|}{ Subject A } & \multicolumn{3}{|c|}{ Subject B } \\
\hline & & $\begin{array}{l}\text { No. } \\
\text { obs. }\end{array}$ & Count & Mean & $\begin{array}{r}\text { No. } \\
\text { obs. }\end{array}$ & $\begin{array}{l}\text { Count } \\
\text { ttllings }\end{array}$ & Mean & $\begin{array}{l}\text { No. } \\
\text { obs. }\end{array}$ & Count & Mean & $\begin{array}{l}\text { No } \\
\text { obs. }\end{array}$ & Count & Mean \\
\hline $400-499$ & May-July & 7 & 110 & $15 \cdot 7$ & 15 & 223 & $14 \cdot 9$ & 18 & 292 & $16 \cdot 2$ & 20 & 315 & $15 \cdot 7$ \\
\hline 500-599 & Aug.-Sept. & 10 & 181 & $18 \cdot 1$ & 13 & 244 & $18 \cdot 8$ & 16 & 240 & $15 \cdot 0$ & 15 & 264 & $17 \cdot 6$ \\
\hline 600-699 & Oct.-Jan. & 36 & 590 & $16 \cdot 4$ & 11 & 145 & $13 \cdot 2$ & 16 & 261 & $16 \cdot 3$ & 8 & 126 & $15 \cdot 8$ \\
\hline 700 & Feb.-Mar. & 11 & 193 & $17 \cdot 5$ & - & - & - & 12 & 198 & 16.5 & - & - & - \\
\hline \multicolumn{2}{|c|}{ Totals } & 64 & 1074 & $16 \cdot 8$ & 39 & 612 & $15 \cdot 7$ & 62 & 991 & $16 \cdot 0$ & 43 & 705 & $16 \cdot 3$ \\
\hline \multicolumn{14}{|c|}{ Wheals } \\
\hline $400-499$ & May-July & 1 & 9 & - & 14 & 165 & $11 \cdot 6$ & 10 & 78 & - & 20 & 223 & $11 \cdot 1$ \\
\hline 500-599 & Aug.-Sept. & 2 & 18 & - & 7 & 114 & $16 \cdot 3$ & 4 & 28 & - & 15 & 199 & $13 \cdot 3$ \\
\hline $600-699$ & Oct.-Jan. & 1 & 9 & - & 10 & 93 & $9 \cdot 3$ & 2 & 14 & - & 8 & 90 & - \\
\hline \multicolumn{2}{|c|}{ Totals } & 4 & 36 & - & 31 & 372 & 11.9 & 16 & 120 & $7 \cdot 5$ & 43 & 516 & $12 \cdot 0$ \\
\hline
\end{tabular}


Biting intensity at age from emergence. An analysis of the records relating to controls carried out on the 5 th, 6th or 7th day from emergence gave the following results.

$\begin{array}{cccc}\begin{array}{c}\text { Days } \\ \text { from }\end{array} & \begin{array}{c}\text { No. } \\ \text { emergence }\end{array} & \begin{array}{c}\text { No. } \\ \text { observed }\end{array} & \begin{array}{c}\text { No. } \\ \text { sheals }\end{array} \\ 5 & 32 & 487 & 306 \\ 6 & 24 & 410 & 265 \\ 7 & 12 & 253 & 175\end{array}$

There is on the average, therefore, according to these figures an increase in the number of settlings, in the number of wheals and in the number of settlings effecting punctures sufficient to show wheals as from the 5 th to the 7 th day from emergence under the conditions as described. In all cases 100 female pupae were originally placed in the emergence cage and any mortality would therefore tend to further enhancement in the biting potential of those remaining.

\section{'Normal feeds'}

In the previous section the number of mosquitoes settling in a period of 15 sec. has been dealt with. A further type of information is given in the case of cages fed for eggs, viz. the rate of settling of a given number of mosquitoes in a cage of the size previously noted ( $0.9 \mathrm{cu} . \mathrm{ft}$.) and the conditions specified, where there is no time limit.

Unfortunately with a large number of mosquitoes such as have usually been fed for stock the number settling is too large to enable any record to be made. In a certain number of smaller feeds, however, attempts have been made with the aid of two observers counting on either side of a line drawn on the arm (in this case fully exposed without shield and with only the hand protected) to keep a record of settlings and in some cases of leavings when gorged. Table 18 gives this information which is also illustrated by the graph (Fig. 6).

The curve of settling as shown by the graph rises in a regular fashion up to the point where mosquitoes begin to leave the arm where a discontinuity is apparent. If, however, the number leaving is added when making the curve there is a fairly steady and regular rise which reaches a maximum at about 5 min., i.e. when almost all the mosquitoes (females) in the cage have settled and are gorging, gorged or have left the arm gorged.

Beyond this point all gradations between two extremes are met with depending upon how far the insects are disturbed. The first act after completion of gorging is to remove the fascicle. Often before doing so the organ is sunk and withdrawn several times in the tissues apparently with no resistance and presumably sliding up and down the 'borehole'.
Final removal is usually accompanied by considerable effort which is communicated as a tremor to the palps and antennae. Usually after withdrawal the proboscis undergoes some extraordinary vermicular-

$\begin{array}{ccc}\text { Mean no. } & \begin{array}{c}\text { Mean no. } \\ \text { wheals }\end{array} & \begin{array}{c}\% \\ \text { wheals to } \\ \text { settlings }\end{array} \\ 15 \cdot 2 & 9 \cdot 6 & 63 \\ 17 \cdot 1 & 11 \cdot 0 & 65 \\ 21 \cdot 1 & 14 \cdot 6 & 69\end{array}$

like movements, probably connected with readjustment of the fascicle in the labial sheath.*

The insect now remains passive with the proboscis directed forwards close to the skin. If undisturbed the insect so remains up to a quarter of an hour or more before flying away. Thus $5 \mathrm{~min}$. or so after introduction of the arm it is largely covered with

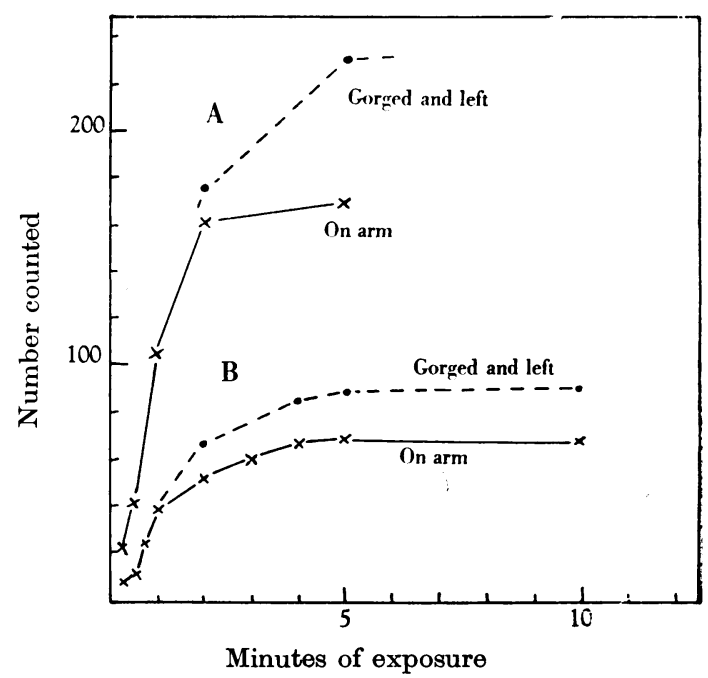

Fig. 6. Graph showing rate of settling in two feeds with (A) 200 and (B) 100 females respectively. The values for the dotted line are superimposed on those given by the unbroken line.

gorged resting mosquitoes that have withdrawn their mouth parts and as these when undisturbed fly away one by one the curve of the count sinks gradually to 0 in about 15 or $20 \mathrm{~min}$. Any disturbing condition may, however, at this stage cause large numbers to leave and the count curve falls abruptly. This part of the curve has therefore little significance.

To the first part of the curve, however, considerable interest attaches since it has relation to the

* It is hoped to publish later bionomical observations on Aedes aegypti which would be out of place in the present connexion. 
Table 18

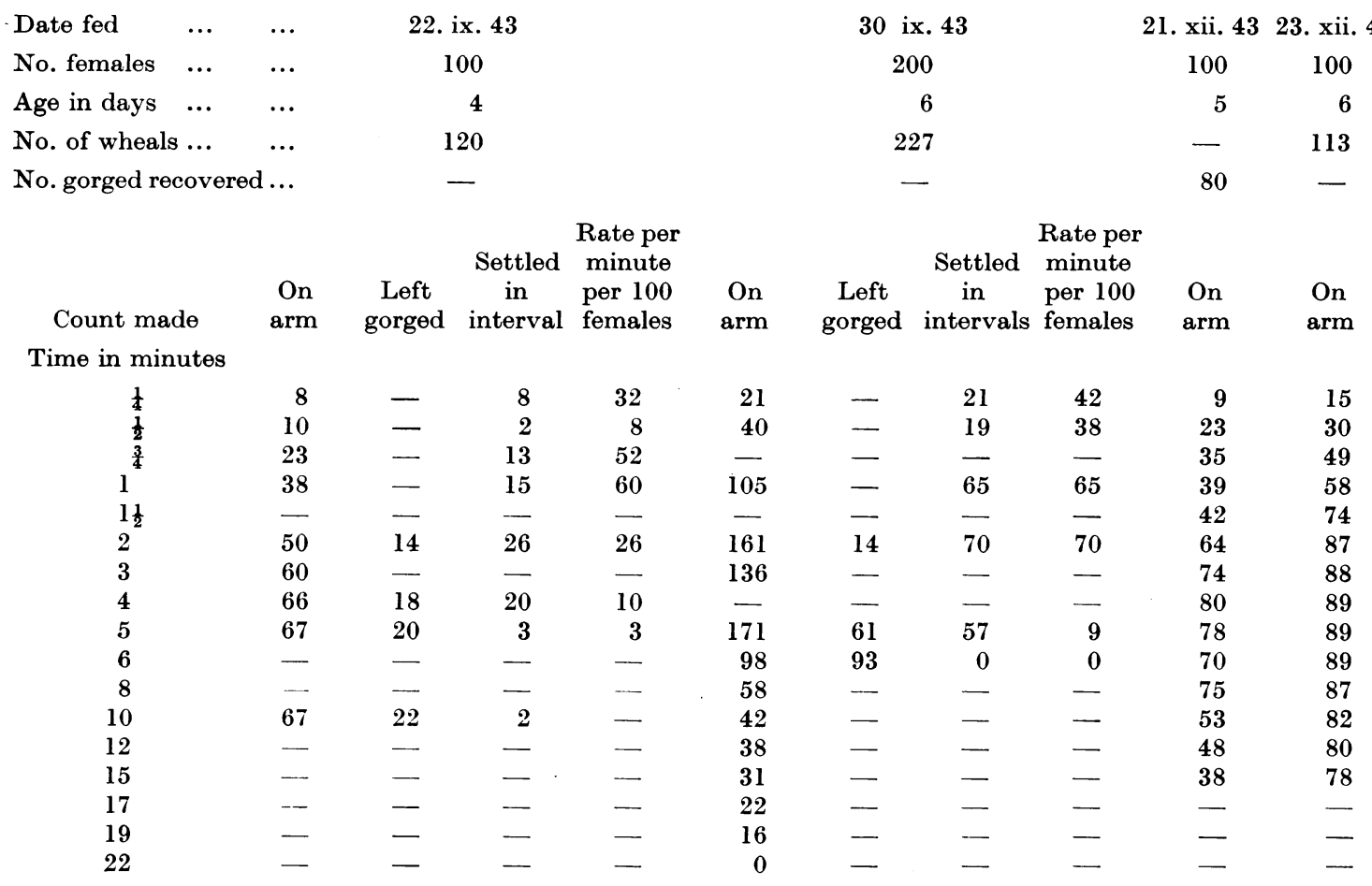

biting rate, an important point in relation to testing. Since under the conditions noted practically all mosquitoes settling remain to puncture and gorge, the settling rate may with sufficient accuracy be considered as the biting rate. It will be evident from the data in Table 15 and the graph that the rate in the first quarter minute in the two counts given is about 30 or 40 per minute. As shown by the 'control' figures it would be 6.4 in 15 sec. on the average or about 60 per minute. This rate continues for from 1 to $2 \mathrm{~min}$. and then rapidly sinks to nil when there are no more mosquitoes to settle. The general rate over the period of $2 \mathrm{~min}$. is then per 100 females about 50.

It is of interest to note that this rapid rate is not confined to what happens in a small cage as used in tests. Single Aedes aegypti liberated into a mosquito net $6 \times 3 \times 6 \mathrm{ft}$. high (108 cu.ft.) in which an observer was seated had settled or were prospecting with the intention of settling within from 2 to 20 sec., independent of the position of the observer or whether all but his head was covered with a white cloth. Alertness to the attack appeared to occur when the mosquito entered a zone (aura) of warm convection currents.

\section{APPENDIX B}

\section{Note by Dr E. A. Moelwyn-Hughes on CONVERSION OF BOILING-POINTS*}

The method of computation is admittedly an approximate one. I have looked into the theoretical basis underlying it, and consider it likely to lead to computed boiling-points differing not more than about $5 \%$ from the true boiling-point, reckoned on the absolute scale. For the purpose of the commentary, this degree of accuracy may prove to be sufficient.

The simplest way of deriving the method is as follows. Let it be assumed that the vapour pressure, $p$, is related as follows to the absolute temperature, $T$ :

$$
\log _{10} p=a-b / T
$$

where $a$ and $b$ are specific constants. This equation is known not to apply over temperature ranges exceeding about $15^{\circ}$. It follows that, if $T$ denotes the temperature at which the vapour pressure is $p \mathrm{~mm}$.,

* Comments made on Commentary No. 20 in the Unit series. It has been very helpful in drawing up the scale given in $\S$ VIII of the Report. 
and $T_{B}$ denotes the boiling-point, at which the vapour pressure is $p_{B}\left(p_{B}=760 \mathrm{~mm}.\right)$, then

$$
\log _{10}\left(\frac{p}{p_{B}}\right)=\frac{b}{T_{B}}-\frac{b}{T} .
$$

According to Trouton's rule we have

$$
b=\frac{K T_{B}}{2 \cdot 303 R}
$$

where $R$ is the gas constant, and $K$ is an approximate constant, having an average value of about 21 cal./g.mol.-degree. Actually, $K$ is as low as 15 for elementary liquids and may be as high as 29 for complicated liquids. Combining equations (2) and (3) we obtain

$$
\frac{T_{B}}{T}=1-\frac{2 \cdot 303 R}{K} \log _{10}\left(\frac{p}{p_{B}}\right) .
$$

Using the numerical values of $R=1 \cdot 987, K=21$, $p=20 \mathrm{~mm}$. and $p_{B}=760 \mathrm{~mm}$., we thus find the following linear relationship between the boilingpoint $T$, at $20 \mathrm{~mm}$. pressure and the boiling-point $T_{B}$ at atmospheric pressure:

$$
T=0 \cdot 74 T_{B}
$$

The gradient given by the experimental points plotted in the graph in the appendix is seen to be 0.77 .

As previously stated, it would thus appear that the method, though approximate, is fundamentally sound, and can lead to computed boiling-points differing by not more than about $5 \%$ from the actual values.

\section{APPENDIX C}

Note by Mr J. R. Whinfield on Elementary CLOTH GEOMETRY*

Cotton fabrics

Sizes of threads expressed as the count $(N)$ :

$840(N)=y \operatorname{ards} / \mathrm{lb} . \quad(N) / 840=\mathrm{lb} . /$ yard.

If $d$ is the diameter of the thread in mils, $\dagger$ then

$$
d=\frac{36}{\sqrt{ }(N)} \text {. }
$$

* I am much indebted to Mr J. R. Whinfield for permission to publish this note which he very kindly gave me for my personal information (see $\S \mathrm{XI}$ of the Report).

$\dagger 1$ mil equals one-thousandth of an inch.
Spacing of threads in the cloth expressed in terms of the number of threads per inch $(t)$.

The number of threads per inch is $1000 / d$, therefore the threads are just touching when

$$
t=\frac{1000}{36 / \sqrt{ }(N)}=28 /(N) .
$$

The value $t / \sqrt{ }(N)$ cannot therefore exceed 28 in absence of compression.

We put $K=t / \sqrt{ }(N), K$ being called the cover factor and expressing the degree of closeness. If $K=14$, then the space between the threads is equal to their diameter.

The following rules are useful:

(1) Cover factors of warp and weft may be kept identical up to a value of about 16. Equality of warp and weft cover factors cannot therefore result in a very close cloth.

(2) A cover factor higher than 16 can be realized in one direction only (warp or weft). This higher value cannot exceed about 32 , at which some compression is involved. Very close cloths are obtained in this way, i.e. by operating to a cover factor in one direction (usually warp) of about 30 .

Specific volume $(V)$. This affords an index of closeness in terms of a single value. If the thickness of the cloth is $G$, then

$$
V=\frac{0 \cdot 75 G}{W}
$$

where $W$ is the weight in oz./sq.yd.

The value of $V$ for cotton itself is $0 \cdot 64$. For threads in a cloth it is of the order of $1 \cdot 1$. The closest possible weaving leads to a value of about 1.3 for cloth. Mosquito proofness demands a value not greatly in excess of this-perhaps 1.5 might be the upper limit, but this is somewhat dependent on the quality and regularity of the threads employed. The sum of the diameters of the threads ( $D$ in mils) is generally a good approximation to $G$ in the case of plain and Oxford woven cloths, but is usually less than $G$ for twills for which quite roughly $1 \cdot 2 D=G$, where $D$ is the sum of the diameters.

Weight $(W)$ expressed in terms of oz./sq.yd. A fair approximation for the relation between weight, spacing of threads and count is given by

$$
W=\frac{S}{N / 0 \cdot 686-(S / 100)^{2}},
$$

where $S=$ the sum of the threads per inch.

\section{REFERENCES}

